\title{
Evidence for distinct serial processes in animals: The multiplicative-factors method
}

\author{
SETH ROBERTS \\ University of California, Berkeley, California
}

\begin{abstract}
The assumption that responses are controlled by distinct serially arranged processes was used by Sternberg (1969a) to explain the result, observed in many human experiments, that two factors have additive effects on reaction time (RT). With slight changes, Sternberg's explanation of additive factors with RT also explains the result, observed in animal experiments, that two factors have multiplicative effects on response rate. This article describes and interprets 17 examples of multiplicative factors from response-rate experiments with rats, pigeons, and goldfish, as well as some other animal evidence for distinct serial processes. The examples suggest and/or support new and old ideas about generalization, attention, timing, learning, motivation, and response production. Most important, the animal evidence makes the case for distinct serial processes considerably stronger. Since the procedures used in the two sets of experiments (human and animal) have little in common, distinct serial processes may control behavior in a very wide range of situations.
\end{abstract}

Sternberg (1969a) introduced the additive-factor method (AFM), a guide to designing and interpreting reaction time (RT) experiments, and used the method to show that there was evidence for distinct serially arranged processes (which Sternberg called processing stages) in a number of human RT experiments. The data that he used to illustrate the method are unusual. They come from factorial RT experiments, and the key result, found in a number of experiments, was that pairs of factors (treatments) had close-to-additive effects on mean RT. One unusual feature of the data is the precision of the additivity. In most cases, an additive model fit so well that in graphs of the data, all of the points (representing the data) touched the fitted lines (representing the model). In a few cases, it was not obvious that the lines had not been drawn through the points. Ten of Sternberg's examples provided enough data to calculate the percentage of variance explained by an additive model; on the average (median over the 10 cases), an additive model describes $99.89 \%$ of the variance. In one set of experiments, not done with the method in mind, an additive model described RTs with median absolute error of $1.8 \mathrm{msec}$ (median taken over five pairs of factors). In an experiment designed for the method, the errors were $.9 \mathrm{msec}$ (one pair of factors) and $.4 \mathrm{msec}$ (same pair at a different level of a third factor), with RTs on the order of $500 \mathrm{msec}$. Sternberg (1971) described 9

This work was supported by National Institutes of Health Grant MH38358. I thank Donald Blough, Russell Church, Peter Eimas, Mark Holder, Leo Postman, Allan Schrier, John Tukey, and Robert Zatorre for comments on the manuscript, and Howard Hoffman, Donald Meltzer, Walter Ritter, and Barry Schwartz for supplying unpublished details of their results. A review by Saul Sternberg contained about 250 helpful comments. Requests for reprints should be sent to Seth Roberts, Department of Psychology, University of California, Berkeley, CA 94720 . more cases in which an additive model fit well; the fits were roughly as close as the fits of Sternberg (1969a). Not only does a simple model fit very well, but the fit is close for a wide range of factors (wide relative to the range of most psychological theories). Sternberg's (1969a) examples included, for example, the factors amount of visual noise, stimulus intensity, stimulus modality, foreperiod duration, length of remembered list, response type (positive or negative), relative frequency of the response, stimulus-response compatibility, and amount of practice. Taking the 1969 and 1971 papers together, there are 20 examples of additivity from 15 experiments, involving 17 different factors, done in 11 different labs.

The combination of close fit, simple fit, and generality across many diverse treatments seems to be unprecedented in the study of behavior. By a simple fit, I mean a fit based on a short and easily understood equation (for examples of this usage, see Tukey, 1977). I have found a few data sets with two of these properties, but none with all three. (Perhaps closeness of fit and simplicity of fit should be considered a single property, because only the presence of both is remarkable.) Fits that are roughly as simple and close as Sternberg's (1969a, 1971) lack generality across treatments. For example, Sternberg's $(1966,1967)$ work on memory scanning found that some RT data could be closely fitted with a straight line (simple fit), but the straight line describes the effect of only one factor, memory-set size. S. S. Stevens found that the results from magnitude-estimation experiments using numerical labeling or cross-modal matching are often linear on log-log plots when intensity is varied, and that, in many cases, all of the data points touch the fitted line (for reviews, see S. S. Stevens, 1966, or Poulton, 1968; for examples of the data, see J. C. Stevens \& Mack, 1959, or J. C. Stevens \& S. S. Stevens, 1960). The procedure of an experiment can be divided into (1) the situation (what re- 
mains constant) and (2) the treatment or factor (what is varied). The power-law result has impressive generality across situations; for example, it has been found with a wide range of stimuli. But it has little generality across treatments; all of the straight lines describe the effect of what can be considered a single factor-stimulus intensity. Likewise, Weber's law-a straight line-is a close description of a great deal of data, from a very wide range of situations, but the factors modeled by the straight line are not diverse: all of them can be considered changes of size, amount, or intensity. A colleague of mine, when asked for an example of data that could be closely fitted with a simple and general model, said, "ROC curves." In some cases, a straight line does pass through all the points of a signal-detection experiment after the data are given a $z$-score transformation (Green \& Swets, 1974; Swets, 1964, 1986). Again, the range of situations is wide, including both human and animal subjects. However, the factors modeled by a straight line have only a little diversity (changes in signal probability, changes in payoff); all of them presumably act in the same way, by changing one's criterion. In most cases, fits as close as the AFM fits are both less simple and less general. For example, the response-rate data of Roberts (1981) are closely fitted with a curve that is the sum of the Gaussian-shaped curve and a ramp-shaped curve. The fit is relatively complex, and only one factor is modeled.

Should a combination of precision, simplicity, and generality across treatments impress us? If so, how much? Most people believe that the probable importance of a theory depends in part on the quality (e.g., precision) of the supporting data (e.g., Freedman, 1983); other things equal, a theory supported by "strong" data deserves more attention than a theory supported by "weak" data. The unprecedented nature of Sternberg's data make it hard to assess. To find data sets similar to Sternberg's within the life sciences, I looked through undergraduate biology textbooks. I found only one: the results of Mendel (1865/ 1966) and his rediscoverers. Given the impact of Mendel's results, and the many differences between plant genetics and human psychology, the comparison may seem far-fetched, but it seems to be the only guidance available. Mendel's experiments, of course, involved the controlled crossing of inbred lines of peas. In some experiments, the important result was the 3:1 ratio of dominant trait (e.g., yellow seed) to recessive trait (green seed). This ratio was found with considerable precision; over seven experiments, the mean ratio was $2.98 \pm .05$ ( $S E$ based on between-experiment variance). Two other experimenters independently found a 3:1 ratio with similar precision (Correns, 1900/1966; De Vries, 1900/1966; this suggests a problem with Fisher's, 1936/1966, conclusion that Mendel's results were too precise). The fit involved here is roughly as simple as Sternberg's-a child who understood subtraction could understand the important feature of Sternberg's results, and a child who understood division could understand the important feature of Mendel's. The generality across treatments of Sternberg's data corresponds to the generality across traits of Mendel's. Mendel found the same results with seven different plant dimensions; later workers added many more examples (Bateson, 1913). It is interesting that the theories of Sternberg and Mendel also have similarities: both involve the combination of distinct abstract functional units. These similarities prove nothing, of course, but they may make Sternberg's work more interesting.

This paper is based on a behavioral data set-response rates measured in a number of previously published animal experiments-that resembles Sternberg's RT data: The rates can be closely fitted in a simple way that describes the effect of a number of qualitatively different factors. Moreover, the main ideas that Sternberg used to explain his RT data-in particular, the assumption of distinct serial processes-also explain, with slight modifications, the response-rate data. The main point of this paper is that the assumption of distinct serial processes, used by Sternberg $(1969 a)$ to explain one set of data-additive factors observed in human RT experiments-also explains another set of data-multiplicative factors observed in animal response-rate experiments and some other animal data-collected under much different conditions.

In spite of Sternberg's data, theories in terms of distinct serial processes have been criticized as implausible (Broadbent, 1984a, 1984b; Grice, Nullmeyer, \& Spiker, 1982; Vaughn \& Ritter, 1973), and the use of RT additivity to infer distinct serial processes has been questioned (Broadbent, 1984a, 1984b; McClelland, 1979; Pachella, 1974; Pieters, 1983; Taylor, 1976; Theios, 1973; Townsend, 1984; Wickelgren, 1977, 1981). More generally, theories that assume distinct serial processes seem to be unpopular (Gardner, 1985, pp. 122-124; Glass, Holyoak, \& Santa, 1979). The animal data strengthen the case for distinct serial processes in a number of ways. Perhaps the most impressive fact is that the animal data have the same rare feature-a simple fit closely describes the effect of a wide range of treatments-previously found only in Sternberg's data. Because the two data sets could have the same explanation, they almost surely do have the same explanation; if they had different explanations, it would be something like lightning striking twice in the same place for different reasons. In addition, the animal data considerably increase the range of the evidence-and, by implication, the range of situations in which distinct serial processes may be important. The procedures used with the animal data seem to have nothing in common with the procedures used with the human data, except for the use of vertebrate subjects and the measurement of brief responses (e.g., leverpress, spoken word).

The next section, which emphasizes reasoning, describes some of the new data and the theory (set of assumptions) that explains it; the reasoning is called the multiplicative-factors method (MFM). The third section ("More Examples of Multiplicative Factors") emphasizes applications and substantive conclusions, and shows the range of data that the theory explains. The final section ("Connections With Other Work') describes other evi- 
dence for distinct serial processes in animals, and discusses similarities and differences between this work and related work.

\section{THE MULTIPLICATIVE-FACTORS METHOD}

\section{A Simple Theory}

The data to be explained come from response-rate experiments with factorial designs. One example is an experiment by Clark (1958) that varied reward schedule and deprivation time. The subjects were 9 rats. They were trained to press a lever for food, then divided into three groups with different schedules of reward: variable interval (VI) $1 \mathrm{~min}$, VI $2 \mathrm{~min}$, and VI $3 \mathrm{~min}$. All groups were fed at the same time each day and tested at seven different times: $1,3,5,7,10,20$, or $23 \mathrm{~h}$ after being fed. Figure 1 shows the response rates from each condition. Not surprisingly, increasing the density of reward raised response rate, and so did increasing deprivation time.

The interesting feature of these results is how the two factors combined. Study of Figure 1 shows that the rates produced by one schedule are close to a constant proportion of the rates produced by the other two; for example, the VI 2-min rates are roughly $75 \%$ of the VI 1 -min rates, regardless of deprivation time. This is more easily seen in Figure 2, where the rates produced by VI $2 \mathrm{~min}$ and VI $3 \mathrm{~min}$ are plotted as a function of the rates produced by VI $1 \mathrm{~min}$. Straight lines constrained to pass through the origin were fitted to the data (Appendix A describes the method of fitting). The deviations from the lines are small and irregular, showing that the two factors- schedule and deprivation time-had multiplicative effects, that is, a change in one factor had the same proportional effect regardless of the level of the other factor.

The multiplicative relation shown in Figure 2 is explained by the theory shown in Figure 3. It assumes that the mechanism that generates leverpresses consists of two serially arranged processes. The first process generates pulses, which travel to a filter. Each pulse that passes through the filter produces a response, which is recorded. The observed response rate $(r)$ is thus the product of the pulse-generation rate $(g)$ and the probability that a pulse is transmitted by the filter $(p)$ :

$$
r=p * g .
$$

The two factors have multiplicative effects on $r$ because one changes $g$ but not $p$, whereas the other changes $p$ but not $g$. Note that this theory explains multiplicative effects with a scale of rate, but not with nonlinear transformations of rate (e.g., log rate).

For concreteness, Figure 3 has deprivation time change the pulse generator $(g)$ and schedule change the filter $(p)$, but, as far as the data are concerned, the assignments of factor to process could just as well be reversed (deprivation changes $p$, schedule changes $g$ ).

More explicitly, a theory that explains Clark's results makes the following assumptions:

1. Distinct serial processes. The mechanism that generates responses can be divided into two distinct serial processes, call them $A$ and $B$. They are distinct, in the sense that each can be changed

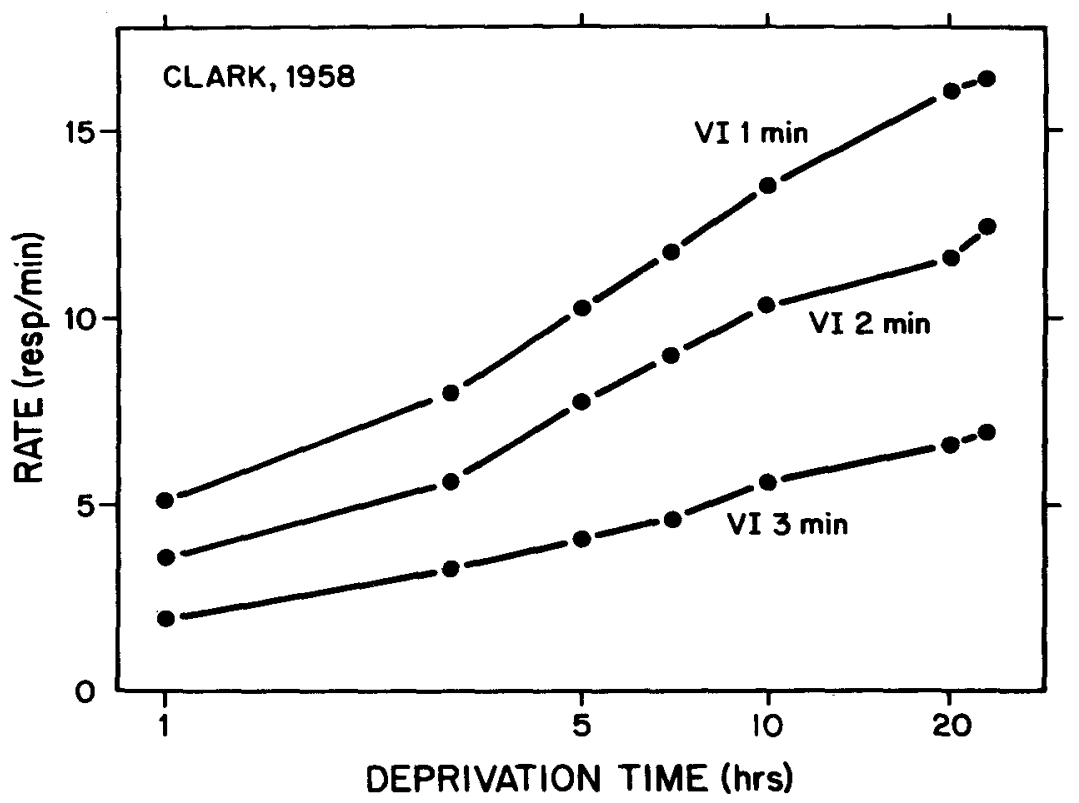

Figure 1. A standard way of plotting the results of Clark (1958), showing response rate as a function of food-deprivation time, for different reward schedules. (Based on Figure 1 of Clark, 1958. Each rate is a mean over three rats. The scale of deprivation time is logarithmic.) 


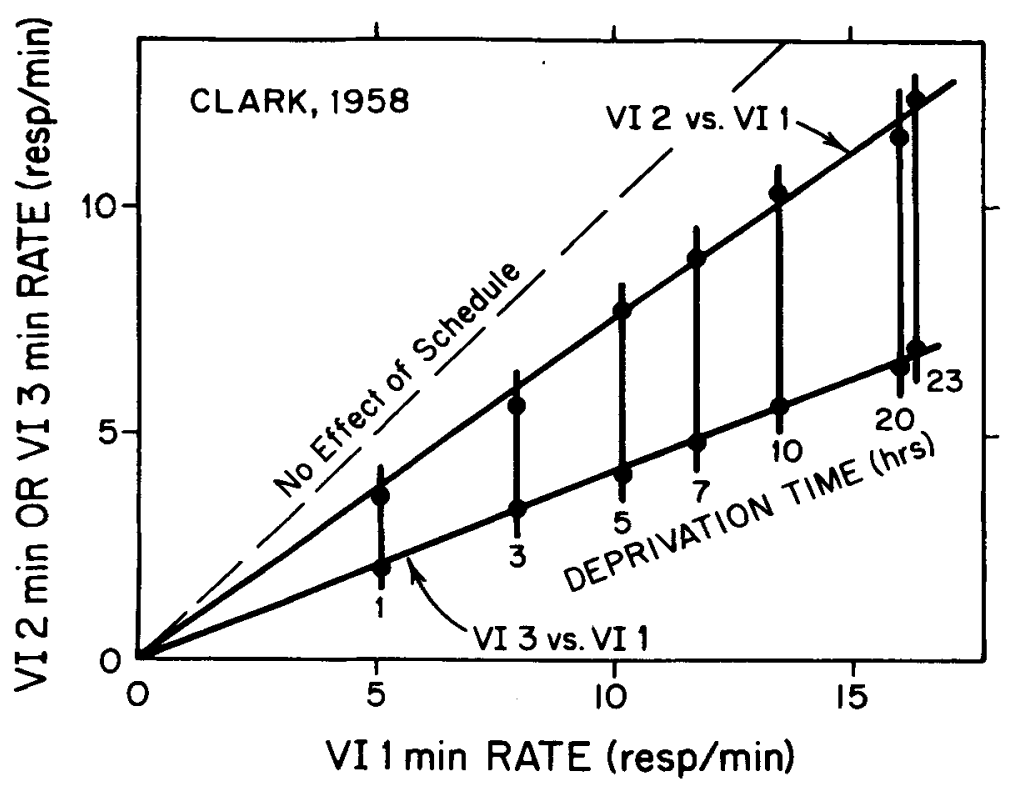

Figure 2. A different plot of the data of Figure 1, emphasizing the multiplicative relation. Rate with VI 2 or VI 3 shown as a function of rate with VI 1, for different values of deprivation time. The fitted lines were determined by the program MULTFTT of McNeil and Tukey (1975), with weighting constant $=9$. They are constrained to go through the origin. For another way of emphasizing the multiplicative relation, see Figure 13.

without changing the other (Roberts, 1981), and serial, in the sense that each response requires (is preceded by) a signal from $A$ to $B$.

2. Countable signals. The signals from $A$ to $B$ can be counted.

3. Selective influence (Sternberg, 1969a). One factor changes $A$ but not $B$, whereas the other factor changes $B$ but not $A$.

4. Uniform signals (as far as $B$ is concerned). Changes, if any, in the nature (e.g., shape, amplitude) of the signals sent from $A$ to $B$ do not change the transmission probability of $B$.

5. Stochastic independence. Changes in the signalgeneration rate of $\boldsymbol{A}$ during a treatment condition are independent of (uncorrelated with) changes in the transmission probability of $B$.

Assumptions 1 and 2 imply that Equation 1 is true over any span of time when $p$ and $g$ are constant. Assumption 5 is necessary to ensure that Equation 1 is true when $p$ and $g$ vary within a condition (e.g., within a session). Assumptions 3 and 4 together imply that the two factors have selective effects on $p$ and $g$. This implies that the two factors will have multiplicative effects on response rate, that is, that data will be fit by a model of the form

$$
r(i, j)=a(i) * b(j),
$$

(Model 1)

where $r(i, j)$ is the response rate at level $i$ of one factor and level $j$ of the other factor, $a(i)$ is the signal-generation rate of $A$ at level $i$ of one factor and $b(j)$ is the transmission probability at level $j$ of the other factor. If Model 1 fits perfectly, the rates will fall on straight lines that cross the origin when plotted as in Figure 2.

Because the signals from $A$ to $B$ are countable, they will be called pulses, and $A$ will be called a pulse generator. Because every response requires a pulse from $A$ to $B, B$ will be called a filter, as if $B$ transmitted only some pulses and transmitted pulses were recorded as responses. Filter

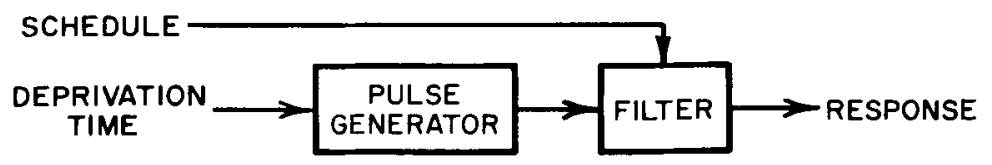

Figure 3. A processing theory suggested by the multiplicative effects shown in Figure 2. Arrows from a factor to a process mean that the factor influences the process. Time proceeds from left to right. Factors are to the left of the processesSternberg's diagrams put them above the process that they influenced-because they can be thought of as experimental stimuli, and because they are present before their effect. 
is used for concreteness. Other possibilities are that $B$ counts pulses and generates a response when a certain sum is reached, or that each pulse from $A$ to $B$ generates more than one response.

\section{A Less Simple Theory}

In many cases, the data are a little more complicated than those of Figure 2-the points fall on a line, but the line does not cross the origin. Figure 4 shows two examples, from two similar experiments by Meltzer and Brahlek $(1968,1970)$. Both experiments involved rats' pressing a lever for food on a fixed-interval (FI) 3-min schedule; in both, the size of the reward was either one or three pellets of food. In one experiment (1968), reward size was a between-subjects factor: Different groups of rats got different sizes. In the other experiment (1970), reward size was a within-subject factor, signaled by the houselight: When the chamber was dark, the reward was one pellet; when it was lit, it was three pellets.

The fits of Figure 4 (see Appendix A for the method of fitting) are close, and the deviations are not systematic (see Appendix B); however, the fitted lines do not pass through the origin, as predicted by the simple theory described earlier. That the $y$-intercepts of the fitted lines are really nonzero is suggested both by the similarity of the intercepts of the two lines and by Meltzer and Brahlek's (1970) data for individual rats (Meltzer, personal communication, January 1977). Straight lines fitted to the data for each rat separately have a mean $( \pm S E$ based on between-subjects variance) intercept of $1.4 \pm .6$ responses per minute (resp/min), significantly nonzero $[t(4)=2.3$, one-tailed $p<.05]$. Unless otherwise stated, all $p$ values are two-tailed. It is the similarity between the results of the 1968 and 1970 data that makes a one-tailed $p$ seem appropriate here. The intercept of the Meltzer and Brahlek $(1968)$ data is not reliably nonzero $[\min t(2)=1.7]$ (see Appendix B).

What could produce a nonzero intercept? The most plausible answer seems to be: Responses produced by a parallel process (parallel to the serial processes that produce multiplicative factors). If so, response rate is a sum, the sum of responses produced by (1) the path suggested by the multiplicative factors (two serial processes) and (2) a parallel path. To preserve linearity, the parallel path must be unchanged by the factor responsible for spacing the points (in Figure 4, time). Responses produced by the parallel path will be called noise, added to the "signal" produced by the two distinct serial processes. In the cases considered here, noise can be thought of as operantlevel responses or as responses not under stimulus control. In Figure 4, the noise would be responses not under the control of time into the FI.

Figure 5 shows the effect of noise on a graph of the results. Suppose that, without noise, the observed response rates lay on a line that passed through the origin. When noise was added, of course, the observed response rates would increase. The noise can fall into one of three categories:

1. Constant with both $\mathrm{F}$ and $\mathrm{G}$ ("Noise unchanged by $F$ and $G$ '). That is, the same amount of noise

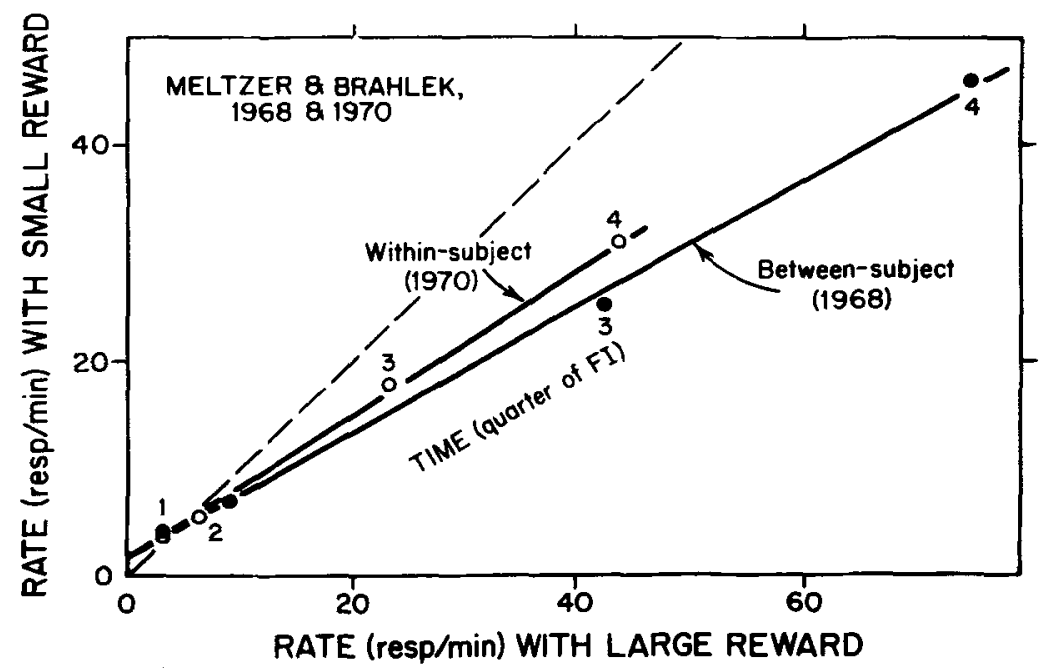

Figure 4. Reward size and time were multiplicative factors. Response rate with a small reward shown as a function of response rate with a large reward, for different parts of the fixed interval. Numbers next to data points label the quarter of the 3 min FI from which the data were recorded; for example, the points labeled "1" come from Seconds 0-45. The 1968 data are from Meltzer and Brahlek (1968, Table 1); the 1970 data are from Donald Meltzer (personal communication, January 1977 ). The 1968 rates are means over 5 rats; the 1970 rates are geometric means over 5 rats. Solid lines were fit with the APL program ROMUFIT of McNeil and Tukey (1975), with weighting constant $=9$. For measures of the goodness of fit, see Table 1 . The broken line is $y=x$, the line of no effect of reward size on response rate. 


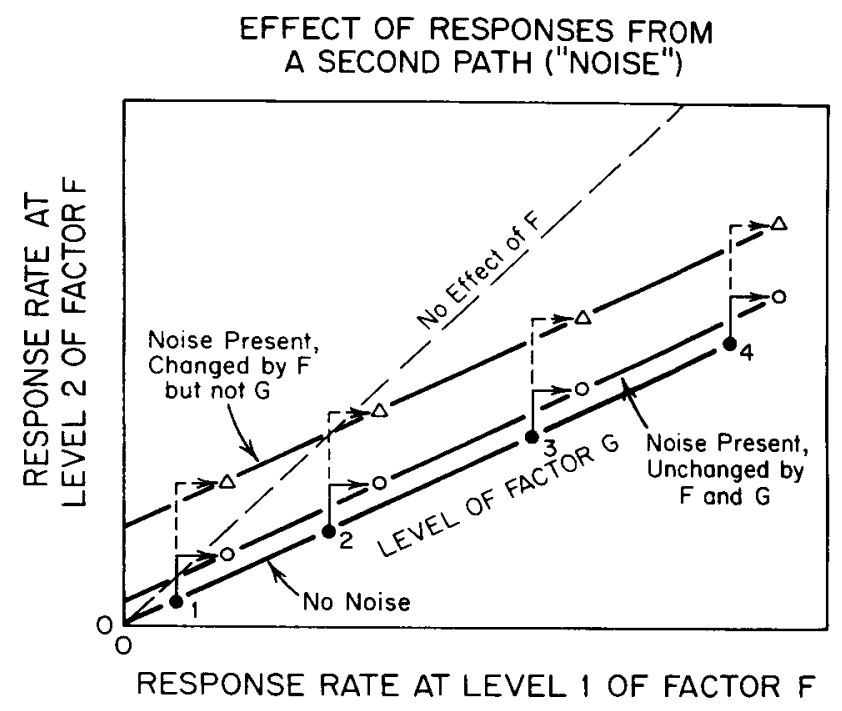

Figure 5. Adding noise causes the rate-versus-rate function to have a nonzero intercept. As long as noise is constant with Factor G, linearity is preserved. The broken line is $y=x$, the main diagonal. If noise is constant with both $F$ and $G$, all data points remain on the same side of the main diagonal and the point where the rateversus-rate function crosses the main diagonal indicates the amount of noise.

is added to each response rate. Then (a) the rates will remain linear, (b) the line will have a positive $y$ intercept, (c) the amount of noise added will be indicated by the $x$ (or $y$ ) coordinate of the point at which the line crosses the main diagonal (labeled "No Effect of $F$ "), and (d) all of the data points will lie on the same side of the main diagonal.

2. Constant with G but not F ("Noise changed by $F$ '). That is, different amounts of noise are added at different levels of $F$. Then (a) the rates will remain linear, but none of the other three conditions (b, c, and d) is necessarily true. For example, the data points may lie on both sides of the main diagonal (as in Figure 4).

3. Not constant with $\mathrm{G}$ (not shown in Figure 5). Then the rates may not remain linear.

In Figure 4, therefore, the nonzero intercepts can be explained by the presence of noise. Because the points of Figure 4 lie on both sides of the main diagonal, noise, if present, varied with reward size. These considerations lead to the theory of Figure 6, which assumes that responses are generated by two different paths. One path, the main path, contains two serial processes (pulse gener- ator and filter). One process is changed by time but not reward size; the other process is changed by reward size but not time. Responses produced by this path somewhere become indistinguishable (the OR gate) from responses produced by the other path, the noise-producing path, which consists of a process influenced by size but not time.

More formally, the theory of Figure 6 predicts that the following model should fit the data:

$$
r(i, j)=[a(i) * b(j)]+c(i) .
$$

The assumptions that lead to Model 2 are the five assumptions listed earlier plus one more:

6. Noise constant with at least one factor. Any responses generated by another path-a path not containing the distinct serial processes-are produced by a mechanism that is not affected by at least one of the two factors.

This assumption, which increases the range of data that the theory can explain, can only be made when one of the two factors has at least three levels. If both factors have two levels, a graph such as that of Figure 4 has only two data points, and the only testable model is one that assumes no noise, predicting that a line through the points crosses the origin.

With many levels of both factors, one can make assumptions about noise that are less restrictive than Assumption 6. For example, one can assume that noise comes from two paths, one changed by Factor $F$ but not Factor $G$, the other changed by $G$ but not $F$. The one would fit a model of the form

$$
r(i, j)=[a(i) * b(j)]+c(i)+d(j) .
$$

How many conditions are needed to test each model? Suppose one factor has $x$ levels and the other factor has $y$ levels. With Model 1 (which assumes no noise), there are $(x-1)(y-1)$ degrees of freedom $(d f)$ for testing the model. With Model 2 (which assumes that noise is constant with one factor), there are $(x-1)(y-2) d f$ when noise is assumed to be unchanged by the factor with $y$ levels. With Model 3, there are $(x-2)(y-2)-2 d f$ (Emerson \& Wong, 1985; see also Mandel, 1971).

Although a few of the data sets considered here have enough levels of both factors to fit Model 3, I fit Model 2 in all cases. It produced adequate fits-fits with small and irregular residuals-so there was little to be learned from trying Model 3. In some cases, Model 2 could be fit in more than one way: Noise could be assumed to be constant with either factor. In these cases, I chose the assump-

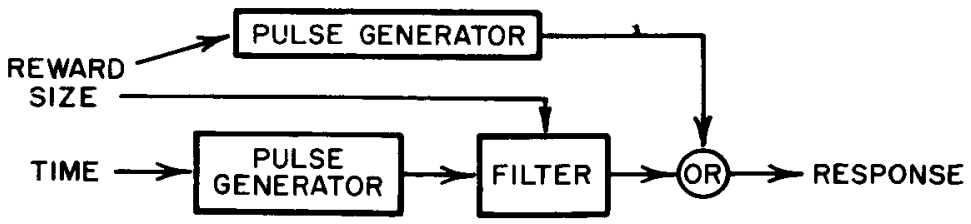

Figure 6. An explanation of the multiplicative effects shown in Figure 4. 
tion that gave the most degrees of freedom. With the Clark data (a $3 \times 7$ experiment), for example, the assumption that noise is constant with deprivation time but not schedule gives $10 d f$ for error; the assumption that noise is constant with schedule but not deprivation time gives $6 d f$ for error. There may be a reason why this choice rule produced close fits-see the end of the section titled "More Examples of Multiplicative Factors."

\section{Alternative Theories}

The theories of Figures 3 and 6 explain the data, but what do other theories predict? This section considers alternatives to each of the assumptions listed earlier. As far as possible, I have tried to derive predictions while changing only one assumption at a time.

1. Both factors change a single process. By itself, this assumption is too vague to make predictions; details are needed. One possibility is that the two factors changed the process (e.g., a pulse generator) in a multiplicative way (e.g., they had multiplicative effects on the pulsegeneration rate). The problem is that the explanation is as detailed as the result.

Another possibility is that the affected process acts like a filter, and the two factors change the threshold of the filter. Suppose that the process receives pulses that vary in some feature (say, amplitude), that all pulses with amplitude above threshold $(t)$ are transmitted and recorded as responses, and that all responses with amplitude below $t$ are not transmitted. With an assumption about the distribution of amplitudes and an assumption about how the two factors affect $t$, this leads to specific predictions. Figure 7 shows the predictions of three variations within

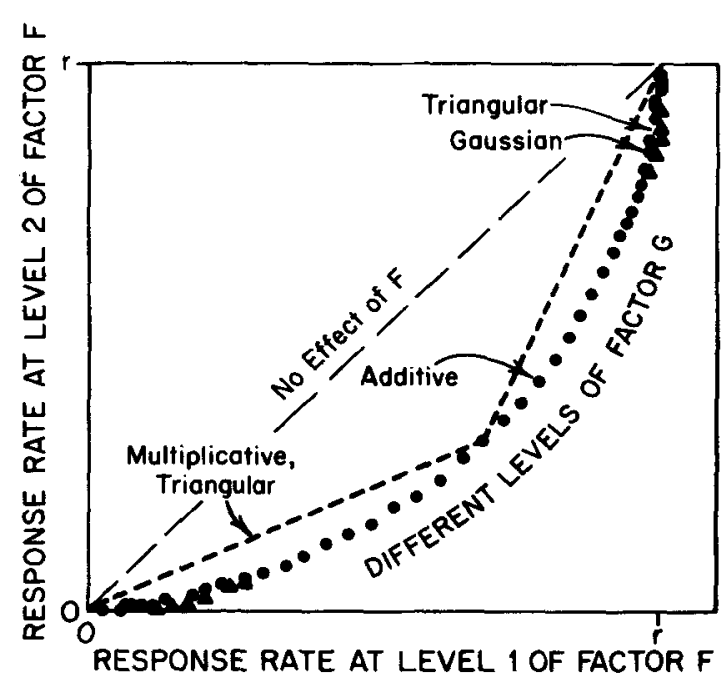

Figure 7. Possible results if two factors change a single process: Rate-versus-rate plots produced by three sets of assumptions. All three sets assume that a response is made when the strength of a signal exceeds a threshold. The sets difier in what assumption is node about the distribution of stganl values-either Gansoinn or triangular-and about the combination rule that describes the effect of the two factors on the threshold-either additive or multiplicative. Signals are generated at rate $R$.
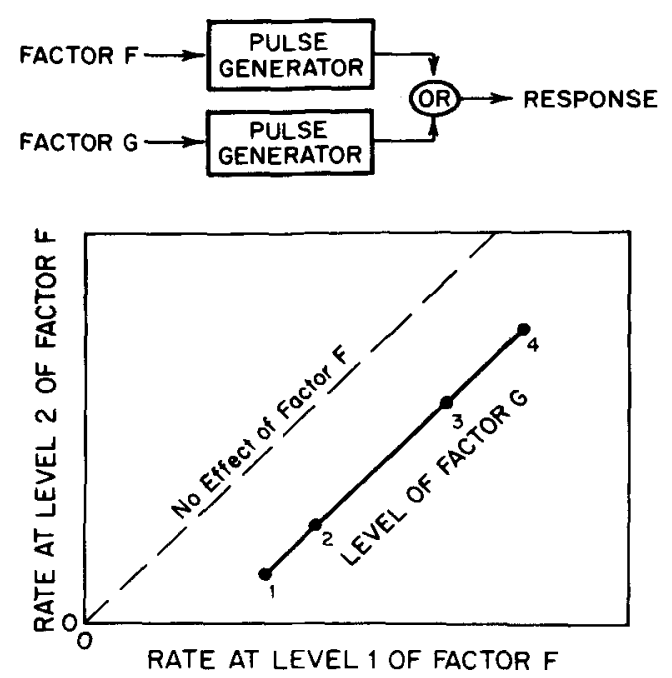

Figure 8. A theory with two distinct processes connected by an OR gate (top) and the prediction that it makes (bottom).

this framework. Two of the variations assume that the factors have additive effects on $t$; one of them assumes a Gaussian distribution of amplitudes, the other a triangular distribution. In both cases, the prediction is that observed rates, when plotted on a rate-versus-rate plot, will lie along a line that is curved and concave upward. The third variation assumes that the two factors have multiplicative effects on $t$, and that the distribution of amplitudes is triangular and becomes nonzero, starting at an amplitude of zero. It predicts that rates will lie on a line that is concave upward with two linear segments. Thus, this possibility could produce multiplicative-appearing factors if the range of the factors were restricted to the lower segment. Again, the problem is that the explanation (assuming multiplicative effects) resembles the result.

2. The two processes are not serial (i.e., a signal from the first to the second is not required to produce a response). One way of combining the output of the two processes is with an $O R$ gate (Figure 8). In this case, a response is produced when either pulse generator generates a pulse. The response rate will be the sum of the pulse-generation rates of the two processes, and two factors that affect different processes will have additive effects on response rate. On a rate-versus-rate plot, this produces a straight line parallel to the main diagonal (Figure 8).

Another way of combining the output of the two processes is with an AND gate (Figure 9). A response is produced only when both pulse generators have generated a pulse. The predictions of this arrangement depend on two parameters: (1) the spacing of pulses from the two generators and (2) the duration of the "hold" of the AND gate-how closely in time two pulses must occur in order to be ANDed together and produce a response. We will consider the extremes of both dimensions: The spacing between pulses is either (1a) completely constant (pulses 
occur at fixed intervals) or (1b) completely random (pulses occur with a fixed probability each moment, so that the number of signals within an interval has a Poisson distribution); and the hold of the AND gate is either (2a) relatively long compared with the time between pulses (e.g., the usual variable-interval schedule, in which reward is primed indefinitely) or (2b) relatively short compared with the time between pulses (so short that there is no overlap between successive holds, so that the probability that a pulse from one generator will produce a response is a multiple of the pulse rate of the other generator).

The four $(2 \times 2)$ possibilities produce three different predictions, which are shown in the graph of Figure 9.

a. The simplest case occurs when the hold is relatively long and signals are generated at fixed intervals. Then the response rate is the minimum of the pulse rates of the two generators. On a rate-versus-rate plot, the results will make a line that goes from the origin up the main diagonal (a segment reflecting no effect of Factor $F$ as long as the generator influenced by Factor $G$ has a lower rate) and then becomes flat (reflecting no effect of Factor $G$ while the generator influenced by Factor $F$ has a lower rate).

b. When holds are relatively long and both generators produce pulses at random intervals, response rate is close to the minimum of the pulse rates of the two generators, but it is less than the minimum because either generator (not just the generator with the higher rate) can produce two signals before the other produces one. The prediction is a curve concave down. See Appendix $C$ for the derivation.
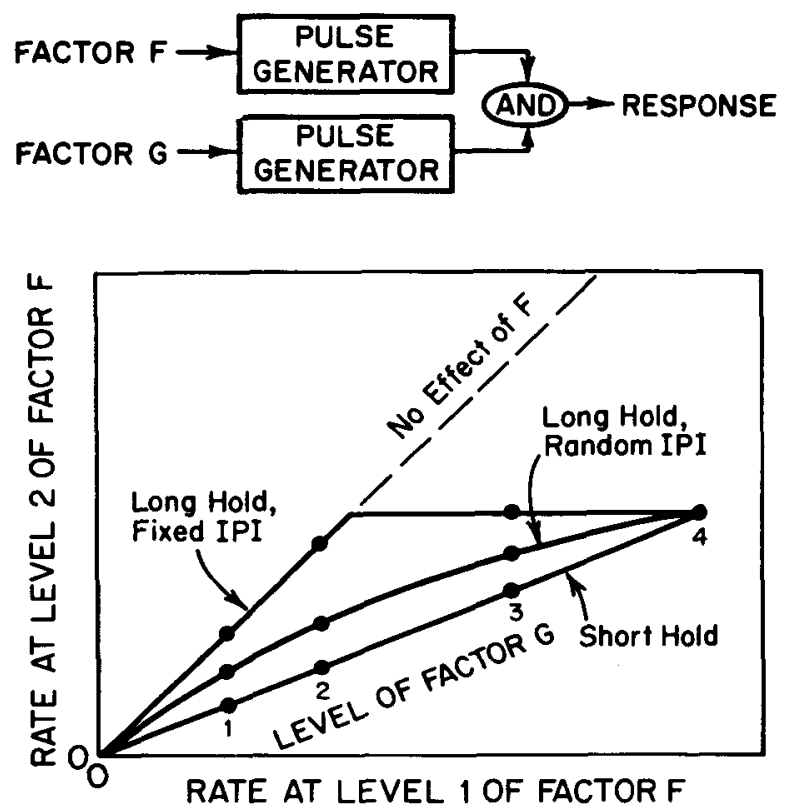

Figure 9. A theory with two distinct processes connected by an AND gate (top) and the predictions that it makes (bottom). The predictions depend on whether the integration period of the AND gate is long or short, and whether interpulse intervals (IPIs) are fixed or random. c. When holds are relatively short, regardless of whether interstimulus intervals are fixed or random, the AND gate, viewed from the perspective of either generator, simply acts as if it is open (transmitting) a certain portion of the time. If the two generators produce pulses independently - more precisely, if the probability that one generator produces a pulse is constant as a function of time since the other generator produced a pulse-then the situation is the same as the simple serial theory (Figure 3 ) considered earlier. Response rate will be a multiple of the product of the pulse rates of the two generators, and thus the two factors will be multiplicative.

3. Signals are not countable. For example, the signal from the first process to the second might be an intensity (e.g., a voltage), with the second process converting intensity to response rate. This arrangement produces multiplicative factors if (a) response rate is a fixed proportion of the signal intensity (fixed across changes in intensity) and (b) the factor that changed the first process changed the intensity and the factor that changed the second process changed the proportion. With almost any other assumptions, of course, this arrangement would not produce multiplicative factors.

4. Signals are not uniform. More precisely, some feature of the signals sent from the first process to the second is changed by the factor that changes the first process, and this change affects the probability of transmission by the second process. This resembles the situation assumed by conventional theories of signal detection (e.g., Green \& Swets, 1974), with a detection process followed by a decision process, and changes in (say) stimulus intensity influencing a feature of the signal sent from the first process to the second. No well-known theory of signal detection predicts multiplicative factors. Some of the possibilities are those considered earlier for the case when two factors change a single process (Figure 7)-notice the resemblance of one of the lines to a receiver operating characteristic (ROC) curve.

5. Lack of independence. Independence is important when both processes vary during a single interval of data collection. Correlation between the two fluctuations can produce artifacts (features of the combined data not found in the data before combination). There are two possible artifacts, shown in the two panels of Figure 10. Both panels show imaginary data from a $2 \times 5$ experiment collected in two sets, A and B, corresponding to different observation times. For example, Set A might be data from the first half of a session and Set B might be data from the second half. The two sets differ in the effectiveness of both factors (called $F$ and $G$ ). In the top panel, for example, the sizes of the factor effects are positively correlated: both factors are more effective in Set $B$.

In both panels of Figure 10, each set (A and $B$ ) taken alone (a) forms a line that (b) crosses the origin, while their arithmetic mean lacks one or both of these features. In the upper panel, the arithmetic mean is linear, but does not cross the origin; in the lower panel, the arithmetic mean is not linear. The two panels differ in whether or 


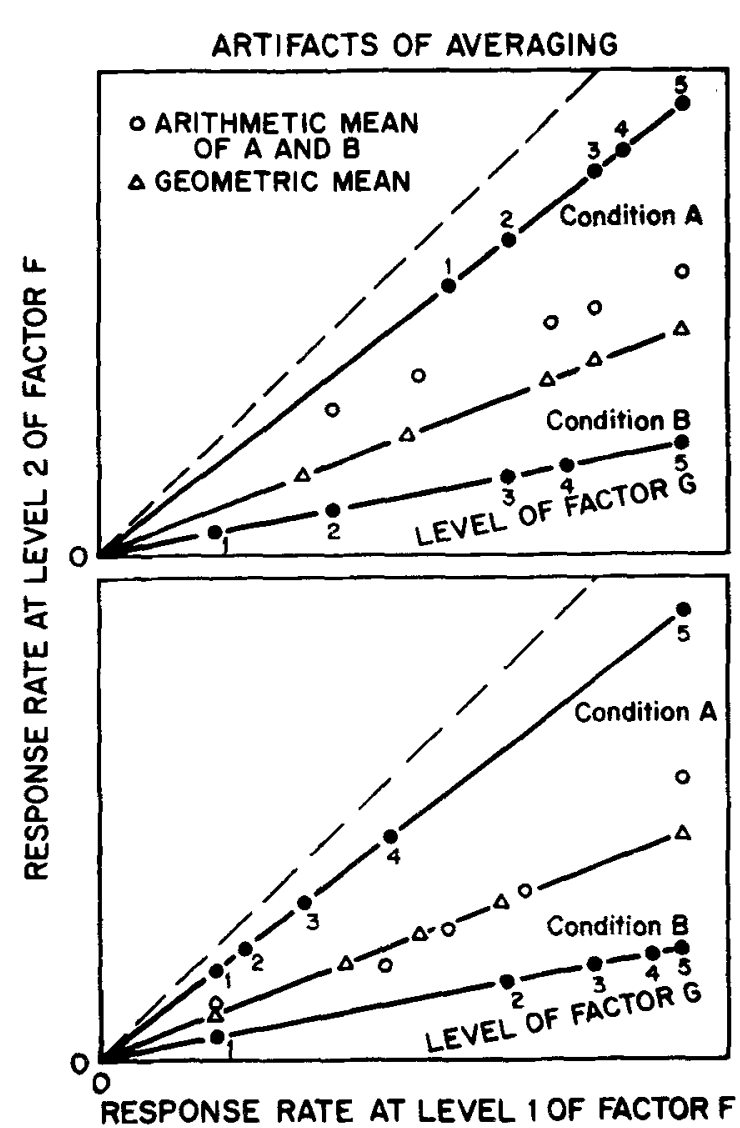

Figure 10. Artifacts of averaging, and the superiority of the geometric mean over the arithmetic mean. Both panels show that the arithmetic means of two data sets, $A$ and $B$, can have properties not present in either of the averaged sets. In both panels, $A$ and $B$ produce linear rate-versus-rate plots, with a zero intercept. In the upper panel, the arithmetic means are linear, but with a nonzero intercept; in the lower panel, the arithmetic means are not linear. In both pancls, however, geometric means of $A$ and $B$ preserve both linearity and the zero intercept.

not Sets $A$ and $B$ have the same relative spacing of the points along the line. In the upper panel, the relative spacing is the same; for instance, the point labeled 2 is onequarter of the distance from 1 to 5 in both Set $A$ and Set $B$. In the lower panel, the relative spacing is different in the two sets.

Figure 10 also shows geometric means, which do not produce artifacts. (Throughout this paper, all rates averaged by me across subjects--such as the data of Meltzer \& Brahlek, 1970, shown in Figure 4-are geometric means.) Unfortunately, the process of collecting cumulative data corresponds to taking the arithmetic mean; for example, the response rate computed from a whole session will be the arithmetic mean of the rates computed from the two halves separately. The fact that geometric means do not produce artifacts is why the assumption of independence is necessary only for variations that occur within a single block of data; variations between blocks of data-for example, from rat to rat-will not cause artifacts if geometric means are used to combine the results.

6. Other assumptions about noise. These have been discussed earlier.

These variations show that if any of the six assumptions is violated, there are many situations in which the two factors would not be multiplicative. So multiplicative factors cannot be considered likely a priori. However, the variations also show that a result of multiplicative factors can be explained in at least five ways:

1. A theory that assumes distinct serial processes and the other five assumptions.

2. A theory that assumes that the two factors changed a single process (its pulse-generation rate or its transmission probability) in a multiplicative way.

3. A theory that assumes that the two factors changed a single process with multiplicative effects on an underlying threshold, and assumes a certain distribution of signal values (triangular) along the dimension of the threshold (Figure 7).

4. A theory that assumes two distinct processes whose output goes to an AND gate with a relatively short hold (Figure 9).

5. A theory that assumes distinct serial processes, but also assumes that the signal from the first process to the second is continuous rather than pulse-like and assumes a specific mapping from the value of the signal to response rate.

But none of the alternatives to Theory 1 seems to be worth serious consideration, at least now. Theory 4 (AND gate) is just a subset - a more detailed version-of Theory 1 (distinct serial processes). In Figure 9, the AND gate is serially arranged relative to the two processes, in the sense that a signal from (say) the upper process to the AND gate is required for a response to be made. The serially arranged processes in Figure 9 are the "boxes" (one process) and the AND gate (the other process). Sometimes Theory 4 may seem particularly plausible (see the Konick and Thomas example below), but it is not a true alternative to Theory 1 . Theories 2,3 , and 5 are true alternatives to Theory 1 , but they lack substance. More or less, they assume what they explain, in the sense that the theory is as detailed as the data. That they explain (or "explain") the data is about as interesting and meaningful as the fact that an equation with five free parameters can perfectly fit five data points (cf. Loftus, 1985). Theory 1 could be wrong, of course, but I have not yet found a substantial alternative explanation of multiplicative factors.

Based on these considerations, it is reasonable to take the observation of multiplicative factors with response rate as support for the six assumptions listed earlier, in particular the assumption of distinct serial processes.

\section{Extension to Subject-Controlled Factors}

The MFM can be extended to interpret experiments in which one of the "factors" is a response feature, controlled by the subject. The basic idea is that if the distri- 


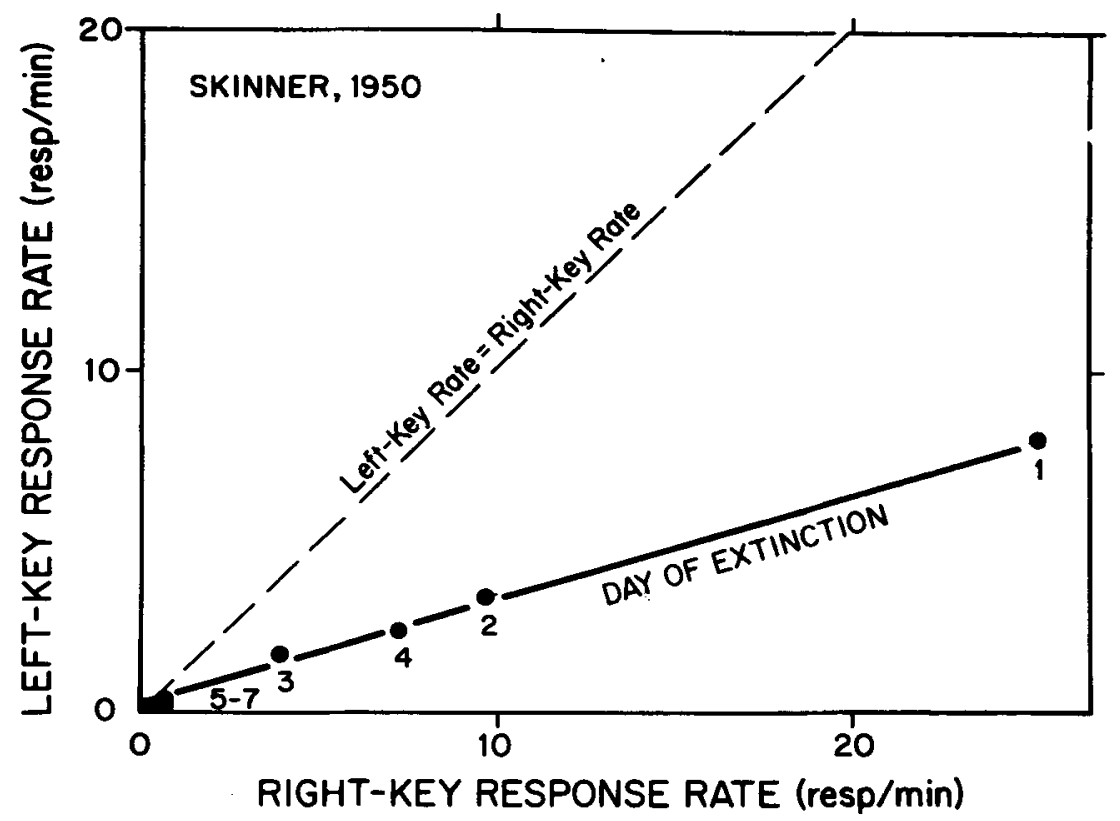

Figure 11. Amount of extinction and key pecked had multiplicative effects. Response rate on the left key shown as a function of response rate on the right key, for different days of extinction. (Based on Skinner, 1950, Figure 13. Apparently from 1 pigeon.) Other details as in Figure 4.

bution of a response feature (e.g., force, latency, amplitude) does not change when the probability of the response changes, this suggests distinct serial processes.

A simple example comes from an experiment briefly mentioned by Skinner (1950). One bird (Skinner does not state the number of subjects explicitly) was trained to peck both the left key and the right key of its box such that the ratio of right-key pecks to left-key pecks was about 3 to 1 . Then all reward was stopped. Seven 1-h extinction sessions, one session per day, produced the data shown in Figure 11. The ratio of left-key responses to right-key responses remained constant while rate decreased. According to Skinner, "several" experiments yielded this result, an unchanging ratio during extinction. Note that rate did not decrease monotonically, suggesting that the data come from one bird.

The results of Figure 11 are explained by the theory of Figure 12. A signal generator produces pulses at a rate that is sensitive to time into extinction. The pulses are sent to a response elaborator, not sensitive to time into extinc- tion, that transforms some of them into left-key pecks and the rest of them into right-key pecks. Time into extinction and key are multiplicative factors (Figure 12) because the rate of left-key (and right-key) pecks is the product of two terms, a rate and a probability, selectively influenced by the two factors. In this case, of course, the response feature with a constant distribution is the ratio of right-key to left-key responses.

The distinct processes suggested by these results are not quite the same as the distinct processes suggested by the earlier applications of the MFM (Figures 3 and 6). One difference is the meaning of distinct. In the earlier examples, the inferred processes are distinct in the sense that each of them can be changed by the experimenter without changing the other; in the Skinner example, the inferred processes are distinct in the sense that the experimenter can change $A$ (the pulse generator) without changing $B$ (the response elaborator), and $B$ varies randomly without changing $A$. (If one measured two response properties at once, and found them uncorrelated, it would suggest a

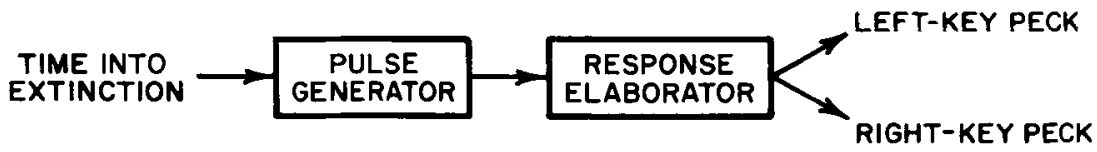

Figure 12. An explanation of the multiplicative relation shown in Figure 11. According to this theory, the amount of extinction influenced the pulse-generation rate. A responseelaboration process, not influenced by the amount of extinction, transformed pulses into either a peck at the left key or a peck at the right key. 
third kind of distinctness, similar to stochastic independence: $A$ varies randomly without changing $B$, and $B$ varies randomly without changing $\boldsymbol{A}$.)

A more important difference is the nature of the inferred processes. With the earlier examples, the second of the two processes can be thought of as a signal condenserit combines information from various sources. In the Meltzer and Brahlek experiments, for example, the second process combines information about duration with information about reward size. The output of the second process (one channel) is less detailed than its input (two channels). In the Skinner example, however, the second process is a signal elaborator-it receives a signal and elaborates it. In this example, it gives the signal (i.e., the response) a location. Its output is more detailed than its input. I will call such processing response elaboration; it is discussed in more detail below (see "More Evidence for Response Elaboration').

\section{An Alternative Graph}

To judge if two factors are multiplicative, the usual way of graphing data (measure vs. treatment, e.g., Figure 1) is less useful than the type of graph (measure vs. measure) shown in most of the data figures (e.g., Figure 2). But a problem with this sort of graph is that when both factors have more than two levels, one level of one of the factors-the factor used to label the axes-will have to be treated differently from the other levels. In Figure 2, for example, treated differently than VI 2 and VI 3. A third type of graph, measure versus part of fit (used by Tukey, 1977, pp. 429, 435) solves this problem.

After fitting the model

$$
r(i, j)=[a(i) * b(j)]+c(i),
$$

one plots $r(i, j)$ versus $b(j)$. Because of the nature of the model, the fitted values will fall on the intersections of criss-crossing lines and the observed values will be directly above or below the intersections. The distances from data point to intersection give the residuals (= data minus fit). As an example, the Clark data (Figures 1 and 2) is shown again in Figure 13. No units or numbers are given for the deprivation-time axis (the $x$-axis) because the units are arbitrary.

With this sort of plot, the assessment of noise is relatively simple. If there is no noise, the tilted lines should intersect at $0 \mathrm{resp} / \mathrm{min}$. If there is a constant amount of noise, the tilted lines should intersect at a single point, at a level equal to the amount of noise. If the amount of noise varies with the factor that distinguishes the radiating lines (in Figure 13, schedule), the lines should not intersect at a single point (if there are more than two lines). In Figure 13, for example, the VI-2 line and the VI-3 line intersect very close to $0 \mathrm{resp} / \mathrm{min}$, and the VI-1 line is, at the point of intersection of VI-2 and VI-3, about .5 resp/ min higher. This has a simple interpretation: The VI-2

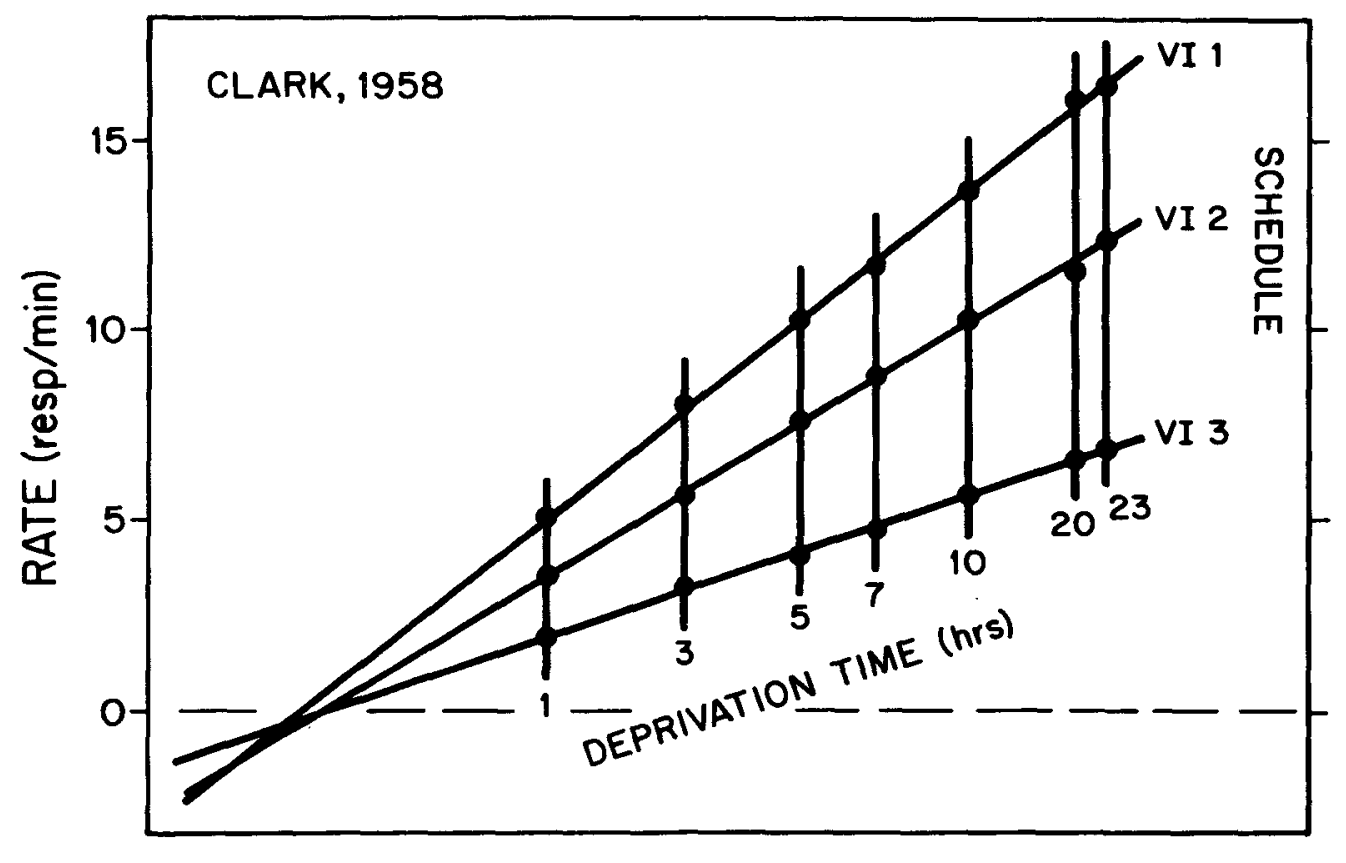

DEPRIVATION-TIME EFFECT

\begin{abstract}
Figure 13. A model-based plot of the data of Figures 1 and 2. Rates are plotted versus the fitted effect of the deprivation time at which the rate was measured. The points where the vertical and sloping lines intersect are the fitted values determined by the APL program ROMUFTT of McNeil and Tukey (1975) with weighting constant $=9$. Differences between the fit and the data are shown by the distance of the data points from the intersections. Measures of the goodness of fit of the model are given in Table 1.
\end{abstract}


and VI-3 schedules produced no noise; the VI-1 schedule produced noise of about $.5 \mathrm{resp} / \mathrm{min}$. Appendix D compares this type of graph to the rate-versus-rate graphs used earlier. This paper uses both types of graphs because each is sometimes better than the other.

\section{Measures of Fit}

Table 1 summarizes the examples of multiplicative factors described in this paper, with one exception (Blough's). To show the range, it lists their subjects and factors. To give some idea of the difficulty of the experiments, it gives the total time (in animal-hours) used to collect the fitted data (e.g., 5 rats for $4 \mathrm{~h}$ each would be $20 \mathrm{~h}$ ), not including the training time before data were collected. The main purpose of Table 1 , however, is to show the closeness of the multiplicative fits. Following McNeil and Tukey (1975), Table 1 gives two measures of goodness of fit. One of them (percent variance left) is almost familiar; it is the percentage of variance not explained by the multiplicative model. In Figure 13, for example, the multiplicative model explains $99.92 \%$ of the variance, so percent variance left $=.08$. This is a measure of fit on what McNeil and Tukey call a "sizesquared" scale; it is conventional, but it does not give a clear impression of the size of the residuals relative to the size of the fit. That is the purpose of the other measure of fit, percent error (recommended by McNeil \& Tukey), defined as $100 \times$ the sum of the absolute value of the residuals divided by the sum of the absolute value of the deviations of the data from their median. If percent error $=2$, for example, the size of the residuals is about $2 \%$ of the size of the fit. The fitting procedure did not specifically minimize either measure of fit.

In four cases, one or two residuals were much larger than the rest; in these cases, the fit of the remaining points is given (both measures of fit lack resistance, i.e., can be drastically changed by a single residual). The omitted data are $3 \%$ of the total data, below many estimates of the frequency of wrong data (Hampel, 1973). The examples in Table 1 are ordered by percent variance left (best fit first), showing that the fit of Figure 13 (99.92\% of the variance) is close to average.

Table 1

Range of Examples and Closeness of Fits

\begin{tabular}{|c|c|c|c|c|c|c|c|c|}
\hline Figure & Experiment & Subjects & Factor A & Factor B & Hours & $d f$ & $\begin{array}{l}\text { Percent } \\
\text { Error }\end{array}$ & $\begin{array}{c}\text { Percent } \\
\text { Variance Left }\end{array}$ \\
\hline 4 & $\begin{array}{l}\text { Meltzer \& Brahlek } \\
\text { (1970) }\end{array}$ & Rats & Time into FI & Reward size & 50 & 2 & 1.5 & .02 \\
\hline 24 & $\begin{array}{l}\text { Roberts (1983), } \\
\text { lever signal }\end{array}$ & Rats & Time into FI & Type of response & 90 & 4 & 2.0 & .03 \\
\hline 21 & $\begin{array}{l}\text { Thomas \& King } \\
\text { (1959) }\end{array}$ & Pigeon & Body weight & Time into extinction & 54 & 2 & 1.0 & .03 \\
\hline 22 & Dinsmoor (1952) & Rats & Body weight & Reward probability & 60 & $4^{*}$ & $1.1^{*}$ & $.03 *$ \\
\hline 4 & $\begin{array}{l}\text { Meltzer \& Brahlek } \\
\text { (1968) }\end{array}$ & Rats & Time into FI & Reward size & 35 & 2 & 2.0 & .04 \\
\hline 16 & $\begin{array}{l}\text { Konick \& Thomas } \\
\text { (1968) }\end{array}$ & Pigeons & Time into FI & Wavelength & 15 & $5^{*}$ & $2.2 *$ & $.04^{*}$ \\
\hline 23 & Miles (1956) & Rats & $\begin{array}{l}\text { Secondary } \\
\text { reinforcement }\end{array}$ & Amount of training & 240 & 10 & 2.0 & .05 \\
\hline 20 & $\begin{array}{l}\text { Azrin \& Holz } \\
\text { (1961) }\end{array}$ & Pigeon & Time into FI & Punishment intensity & 150 & 8 & 3.1 & .07 \\
\hline 19 & Rozin (1965) & Goldfish & Time into FI & Water temperature & 45 & 4 & 2.7 & .07 \\
\hline 13 & Clark (1958) & Rats & Deprivation time & Reward probability & 28 & 10 & 2.6 & .08 \\
\hline 18 & $\begin{array}{l}\text { Roberts (1983), } \\
\text { lever responses }\end{array}$ & Rats & Time into FI & Reward probability & 90 & 4 & 1.7 & .11 \\
\hline 15 & Tomie et al., (1973) & Pigeons & Wavelength & Type of training & 22 & $5^{*}$ & $3.6^{*}$ & $.12 *$ \\
\hline 18 & $\begin{array}{l}\text { Roberts (1983), } \\
\text { chain responses }\end{array}$ & Rats & Time into FI & Reward probability & 90 & 4 & 2.3 & .13 \\
\hline 24 & $\begin{array}{l}\text { Roberts (1983), } \\
\text { chain signal }\end{array}$ & Rats & Time into FI & Type of response & 90 & 4 & 2.5 & .17 \\
\hline 14 & $\begin{array}{l}\text { Hearst \& Koresko } \\
\text { (1968), } \\
\text { White Carneaux }\end{array}$ & Pigeons & Line tilt & Days of training & 34 & 18 & 8.3 & .68 \\
\hline 14 & $\begin{array}{l}\text { Hearst \& Koresko } \\
\text { (1968), White King }\end{array}$ & Pigeons & Line tilt & Days of training & 34 & $16 \dagger$ & $8.5 \dagger$ & $.69 \dagger$ \\
\hline 11 & Skinner (1950) & Pigeons & $\begin{array}{l}\text { Days of } \\
\text { extinction }\end{array}$ & Key pecked & 7 & 5 & 6.2 & .92 \\
\hline \multicolumn{5}{|c|}{ Median $(n=17)$} & 50 & 4 & 2.2 & .07 \\
\hline
\end{tabular}

Note-The examples are ordered by percent variance left. The fitting process assumed that Factor $A$ did not change the amount of noise, but that Factor $B$ might have. The Miles data assumes $30 \mathrm{~min}$ per animal. Hours $=$ observation time used to collect data (doesn't include training time prior to data collection). Percent error is defined in the text. Percent variance left $=100-$ variance described by the fit. *One point not included. †Two points not included. 


\section{MORE EXAMPLES OF MULTIPLICATIVE FACTORS}

This section describes 13 more examples of multiplicative factors, grouped by the conclusion that they suggest. The order of the conclusions is from input (stimulus processing) to output (response processing).

\section{Evidence for Distinct Dimensional Analyzers}

Sutherland and Mackintosh (1971), as part of their theory of attention, assumed that responses were controlled in part by dimensional analyzers whose output went to an associative process that attached the output to responses. One analyzer determined the brightness of the stimulus, another its color, and so forth. Some applications of the MFM support theories along these lines.

In an experiment with pigeons, Hearst and Koresko (1968) measured the effect of amount of training on linetilt generalization. Training consisted of $\mathrm{S}+$ periods ( $\mathrm{S}+$ was a vertical line) alternated with blackouts. Different groups of birds received different amounts of training$2,4,7$, or 14 days' worth. Generalization was measured in extinction, with each bird shown eight test tilts. The experiment was done with White Carneaux pigeons, then repeated with White King pigeons.

Figure 14 shows the results and the multiplicative fits. The fits are not as close as the other examples in this paper, but the residuals appear to be irregular. The White King residuals, by themselves, appear slightly systematic, bowed upward with 14 days of training, bowed downward with 2 days, but the White Carneaux residuals do not show the same pattern.

These results suggest that responding was in part generated by two distinct serial processes, one of them, a linetilt analyzer, sensitive to tilt (perhaps signaling the difference from $S+$ ) but not days of training, the other sensitive to days of training but not tilt (perhaps an amplification process). The failure of the lines to cross near zero suggests that there was noise (responses generated by a mechanism not sensitive to tilt)-in this case, considerable noise. With the White King data, the lines for 2, 4, and 7 days of training cross at about $15 \mathrm{resp} / \mathrm{min}$, suggesting that amount of noise for those three groups. From 2 to 7 days, the predominant effect of training was apparently to increase the amplification of the line-tilt signal (shown by the increase in slope); from 7 to 14 days, perhaps noise increased as well. Turning to the White Carneaux results, the four lines obviously do not intersect at one point, suggesting that the amount of noise varied with the amount of training. That the slopes of the lines increased only slightly from 2 days to 14 days suggests that training increased amplification of the line-tilt signal only slightly; its main effect, apparently, was to increase noise.

Another example comes from a generalization experiment by Tomie, Davitt, and Thomas (1973) that measured the effect of treating two stimuli as equivalent. Pigeons were trained for 20 days to peck an illuminated key, then

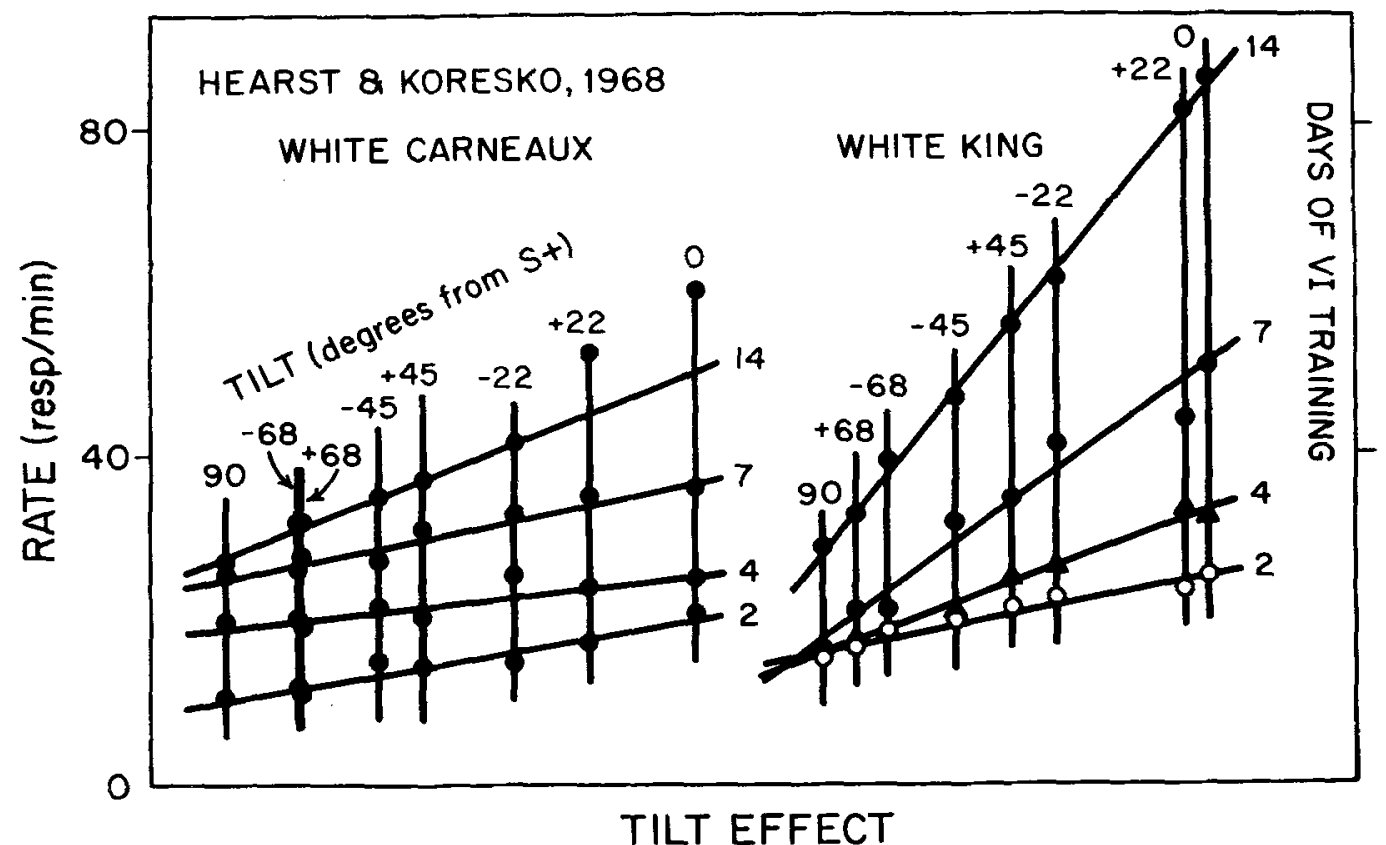

Figure 14. Tilt and amount of training had multiplicative effects. Response rate shown as a function of the fitted tilt effect, for different tilts and amounts of VI training. Based on Hearst and Koresko (1968, Figure 1). The White King data are shifted rightward relative to the White Carneaux data. Different plotting symbols are used for the lower two lines of the White King data to make clear which line the points belong to. Each rate is a mean over 8 pigeons. Other details are as in Figure 13. 
tested for wavelength generalization during extinction. There were three groups of birds, and different groups received different training. Group A, a baseline group, was trained with only one stimulus, a key lit by a light of $555 \mathrm{~nm}$. Group B was trained with the key lit with a $555-\mathrm{nm}$ light and with a white line on a 555-nm background. The two stimuli randomly alternated on one key; the schedule of reward was constant. Group C was trained with the key lit with a 555-nm light and with a white line on a black background. Again, the two stimuli randomly alternated on one key, while the schedule of reward remained constant. For all three groups, the key was constantly lit; there were no intervening blackouts.

The results are shown in Figure 15. With the exception of one point, a multiplicative model fits well. This suggests that responding was controlled by a wavelengthsensitive process followed by a training-sensitive process. (The order of the processes is of course based on other considerations.) The three lines do not meet at one point, suggesting that the difference between groups affected the amount of noise (responses not controlled by wavelength). Assuming that noise was constant with wavelength, noise for Group A must have been between 0 and the lowest measured rate for that group, $2 \mathrm{resp} / \mathrm{min}$ (at $606 \mathrm{~nm}$ ). If noise was $\mathbf{0}$ for Group A, then, by extrapolation, noise would have been about $2 \mathrm{resp} / \mathrm{min}$ for Group B. These considerations suggest that noise was greatest for Group C, least for Group A. Slopes of the fitted lines were in the opposite order-most for A, least for C. In this situation, then, more control by wavelength (i.e., more amplification of the wavelength signal) was correlated with less control by other dimensions (i.e., less noise), a possible example of the "inverse rule" of attention (e.g., Mackintosh, 1975).

Tomie et al. concluded only that training affected the sharpness of relative generalization gradients. Given the model supported by these results-an analyzer followed by an amplifier, plus noise-a change in the sharpness of a relative generalization gradient could be due to (1) a change in the sensitivity of the analyzer; (2) a change in amplification; (3) a change in noise; or (4) any combination of the first three changes. Plots such as those in Figure 15 provide a way of choosing between the seven possibilities. In this case and in the Hearst and Koresko (1968) case, these plots suggest that training changed amplification and noise, but not sensitivity. With simultaneous changes in amplification and noise, no wonder Hearst and Koresko found the usual generalization gradients uninterpretable. Without a way of separately measuring sensitivity, amplification, and noise, no wonder, perhaps, that the study of attention in animals (reviewed by Riley \& Leith, 1976) has been difficult.

The results of Figures 14, 15, 16 (below), and 27 (below) suggest the existence of dimensional analyzers, at least for tilt and wavelength, that are distinct from other processes. The results of Blough (1972, especially Experiments 3 and 4), involving a different pattern of data, seem to suggest the same thing, although not as clearly; the dimensions used by Blough were time since food, tone frequency, line tilt, and wavelength. This conclusion (the

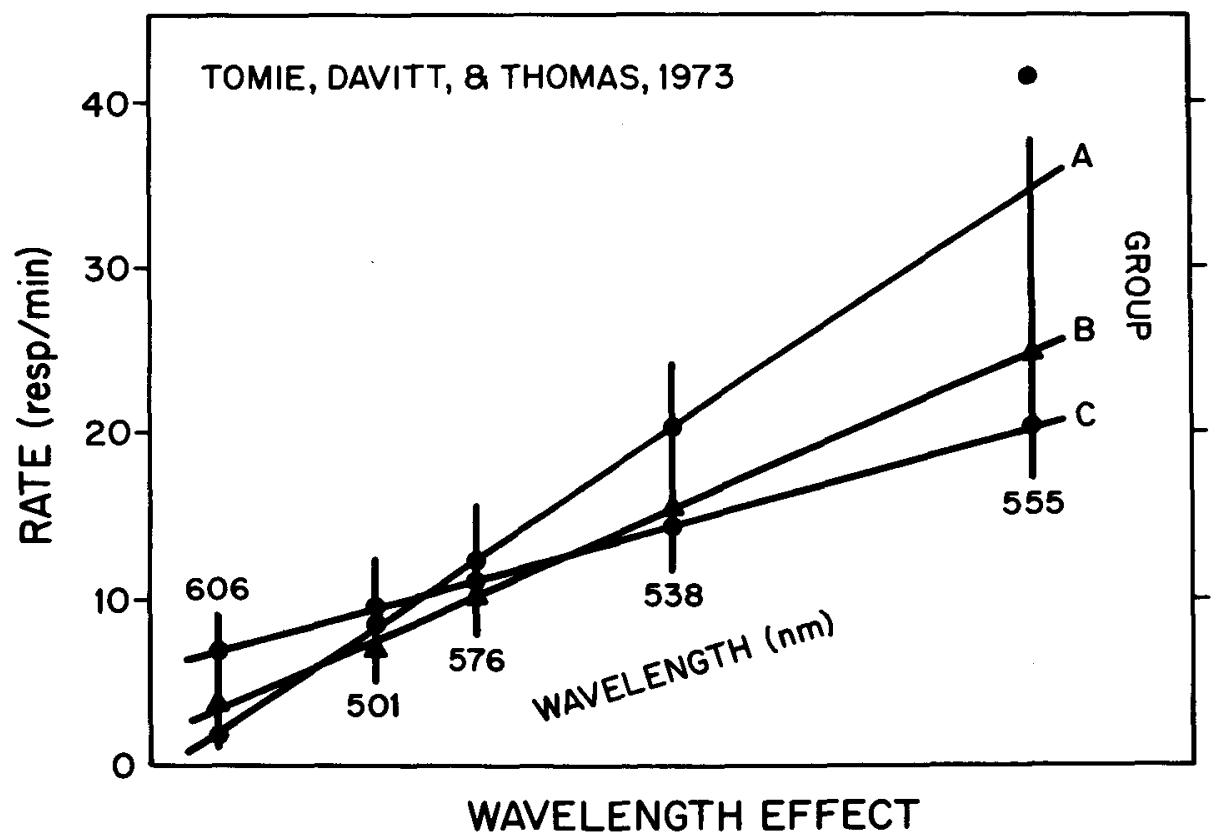

Figure 15. Wavelength and type of training had multiplicative effects. Response rate shown as a function of the fitted wavelength effect, for different wavelengths and types of training. Based on Tomie, Davitt, and Thomas (1973, Figure 1 and text). Different groups received different types of training. A different plotting symbol is used for Group B to make clear which line the points belong to. Each rate is a mean over 8 pigeons. Other details as in Figure 13. 
existence of dimensional analyzers) may be unsurprising because data from electrical stimulation of the cortex (e.g., Penfield \& Roberts, 1959, pp. 23-37), lesions (e.g., M. Wilson, 1957), and single-cell recordings (e.g., Kuffler \& Nicholls, 1976, pp. 16-73) suggest that a substantial part of the vertebrate brain is devoted to sensory analysis, especially visual analysis. Still, the MFM conclusions supplement the physiological conclusions in a number of ways. The MFM results, unlike the physiological work, place the analyzers within a larger context by suggesting that they are linked to other functionally important processes and that they are distinct from other processes that control the same response. The MFM results also suggest new details: that the signal from the analyzers is pulse-like and that variations in the dimension are coded by variations in the rate of signals. A critic might comment that we already know that there are cells in the visual system that vary their firing rate in response to (say) line tilt (e.g., Kuffler \& Nicholls, 1976, pp. 4043). Maybe this makes the MFM conclusions more plausible. Nobody has shown that the firing rate of visualsystem cells has any connection with behavior.

\section{More Evidence for a Distinct Internal Clock}

Some theories of duration discrimination have assumed that responses are controlled in part by a durationmeasuring process-an internal clock-that is distinct from other processes (e.g., Gibbon \& Church, 1984; Roberts \& Church, 1978; Treisman, 1963). The Meltzer and Brahlek results (Figure 4) support this idea, and so do the results of four other experiments:

1. Konick and Thomas (1968) trained 10 pigeons to peck at a 555-nm keylight on an FI 1-min schedule; timeout periods of varying length, with a dark key, followed each completed interval. After $\mathbf{4 0} \mathrm{h}$ of training, the birds were given generalization tests in extinction. One of the tested wavelengths produced almost no responses; results from the other four are shown in Figure 16. Except for one point, the results are close to multiplicative. Including the aberrant point, the model (with $6 d f$ ) describes $99.88 \%$ of the variance; without the odd point ( $5 \mathrm{df})$, it describes $99.96 \%$. The residuals appear irregular. Apparently, time and wavelength changed distinct serial processes. The fact that the sloping lines in Figure 16 do not meet at a single point suggests that noise varied with wavelength. Because both factors had the same number of levels, the rule used with the other examplesmaximize the degrees of freedom-did not specify which factor to assume did not change noise. The choice was made by looking at graphs based on the two possibilities. The fit shown in Figure 16 assumes that noise was constant with time. The fit that assumed noise constant with wavelength produced residuals that appeared systematic.

Blough (1972, Experiment 2) used the factors of time and wavelength in a similar experiment with pigeons. Blough's own analysis suggested that the two factors had effects that were close to multiplicative, but systematically less. If Blough's analysis had allowed for noise, a multiplicative model would perhaps have fit well.

Interpretation of the Konick and Thomas results illustrates the points that (a) the MFM does not order the processes that it suggests, and (b) in some cases no ordering may be possible. The two theories shown in Figure 17 are two possible explanations of the results of Figure 16; for simplicity, both omit an explanation of the nonzero intercepts. Maybe the upper theory is what one would consider first, but it has a problem: Processes that are in some sense the same are treated differently. The

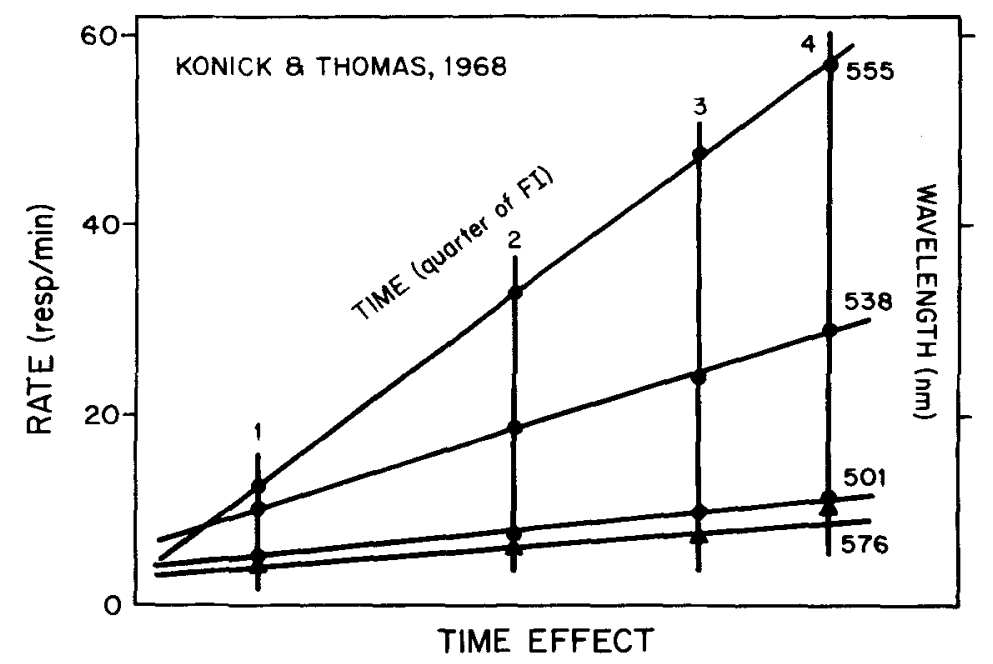

Figure 16. Time and wavelength had multiplicative effects. Response rate shown as a function of the fitted quarter-of-FI effect, for different quarters of the FI and different wavelengths. Based on Konick and Thomas (1968, Figure 1). A different plotting symbol is used for $576 \mathrm{~nm}$ to make clear which line the points belong to. Each rate is a mean over 10 pigeons. Other details as in Figure 13. 

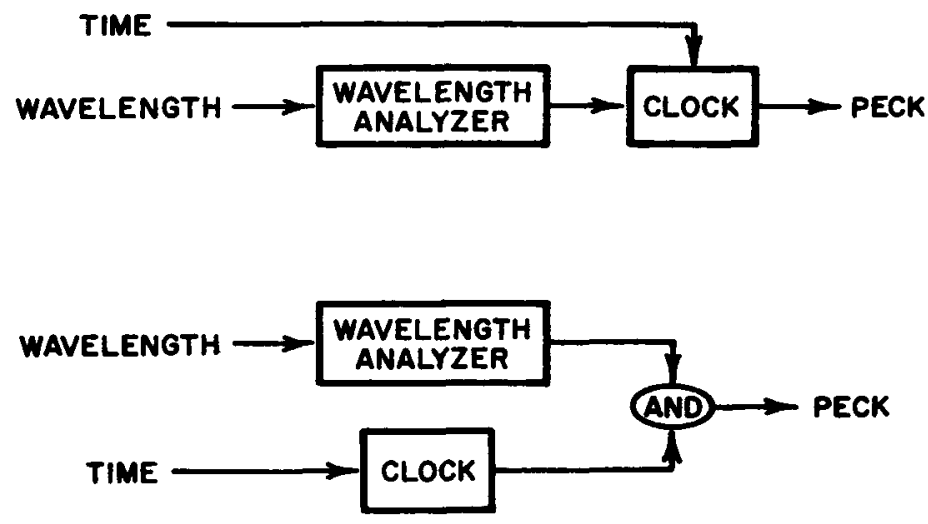

Figure 17. Two explanations of the results of Figure 16.

clock and the wavelength analyzer do essentially the same thing-measure a dimension of the stimulus-and therefore should be equally close to the sensory system, until we know more. This suggests the lower theory of Figure 17, where the output of the two processes is connected by an AND gate. If the lower theory is correct, it would be fruitless to try to decide if the clock was before or after the wavelength analyzer. The serial processes in the lower theory are the clock (or the wavelength analyzer) and the AND gate.

2. Roberts (1983) combined a time discrimination (respond only at a particular time) with a response discrimination (make one response during one signal, another response during another signal). Six rats were trained to respond for food on a discrete-trial schedule, with trials defined by light or sound. Two responses, a leverpress and a chain pull, were always available, but during one signal (light for half the rats, sound for the other half), only lever responses were rewarded, and during the other signal, only chain responses were rewarded. After the response discrimination was established, a time discrimination was added: Food was given only for the first response appropriate to the signal (light or sound) made more than $60 \mathrm{sec}$ after the signal began.

Responses were recorded in bins $5 \mathrm{sec}$ wide, so the 60sec interval was divided into 12 parts. Figure 18 shows the data in a form helpful for deciding if signal and time had multiplicative effects. (The assignments of light and sound to responses were balanced, and the two modalities had almost identical effects-see Roberts, 1983, for details.) For both types of responses, time and signal were close to multiplicative after the first $30 \mathrm{sec}$ of the signal. This suggests that responding was controlled by two distinct serial processes, one sensitive to the duration of the signal and the other sensitive to its modality. Perhaps the multiplicative model fails to fit the data from the first $30 \mathrm{sec}$ of the signal because it took $30 \mathrm{sec}$ for the amount of noise to become constant or for the lever-signal/chainsignal difference to have its full effect. The intercepts are not reliably nonzero $[t s(4)=1.4,0.4]$.
3. Rozin (1965) trained three goldfish to press a lever on an FI 1-min schedule. After the time discrimination was established, the water temperature was changed from $30^{\circ} \mathrm{C}$ to $20^{\circ} \mathrm{C}$. The sequence of conditions -2 days at $30^{\circ} \mathrm{C}, 2$ days at $20^{\circ} \mathrm{C}, 2$ days at $30^{\circ} \mathrm{C}$-was repeated six times. Figure 19 shows the results, taken from a graph in Rozin's paper. Figure 19 gives relative rates because Rozin reported only relative rates. The fit is close, and the deviations are not systematic. This suggests that time and temperature changed distinct serial processes. The intercept is reliably nonzero $[t(2)=6.6, p<.05]$, suggesting that some responses were produced by a process not sensitive to time.

4. The final data suggesting a distinct internal clock come from Azrin and Holz (1961), who measured the effect of punishment on the FI performance of two pigeons. The FI was $5 \mathrm{~min}$. Punishment was a brief electric shock, and every response was punished. They measured the peck rate of one of the birds for 22 months, $4 \mathrm{~h}$ per day. During this time, the voltage of the punishment was varied from 0 to 180 . As the voltage increased, overall response rate went from 30 to 2 resp/min, but the "quarter-life" (the proportion of the interval taken to make a quarter of the total responses) was constant. Figure 20 shows the response rates for most of the conditions. Rates with $30-\mathrm{V}$ punishment are not shown because they were almost identical to the $0-\mathrm{V}$ results; rates with 150 - and $180-\mathrm{V}$ punishment are not shown because they were nearly zero and hard to read from the published graph. A multiplicative model fits well, and the residuals appear irregular. This suggests that time and punishment changed distinct serial processes. The results of the other bird, run for a shorter time and fewer conditions, were consistent with this conclusion but too irregular to place any weight on.

So there is evidence for a distinct internal clock in experiments with pigeons, rats, and goldfish. Moreover, the clock sends (or receives) pulse-like output to (or from) another process. Earlier evidence (Roberts, 1981, 1983) came only from rats, and revealed nothing about the connection between the clock and other processes. Given the 


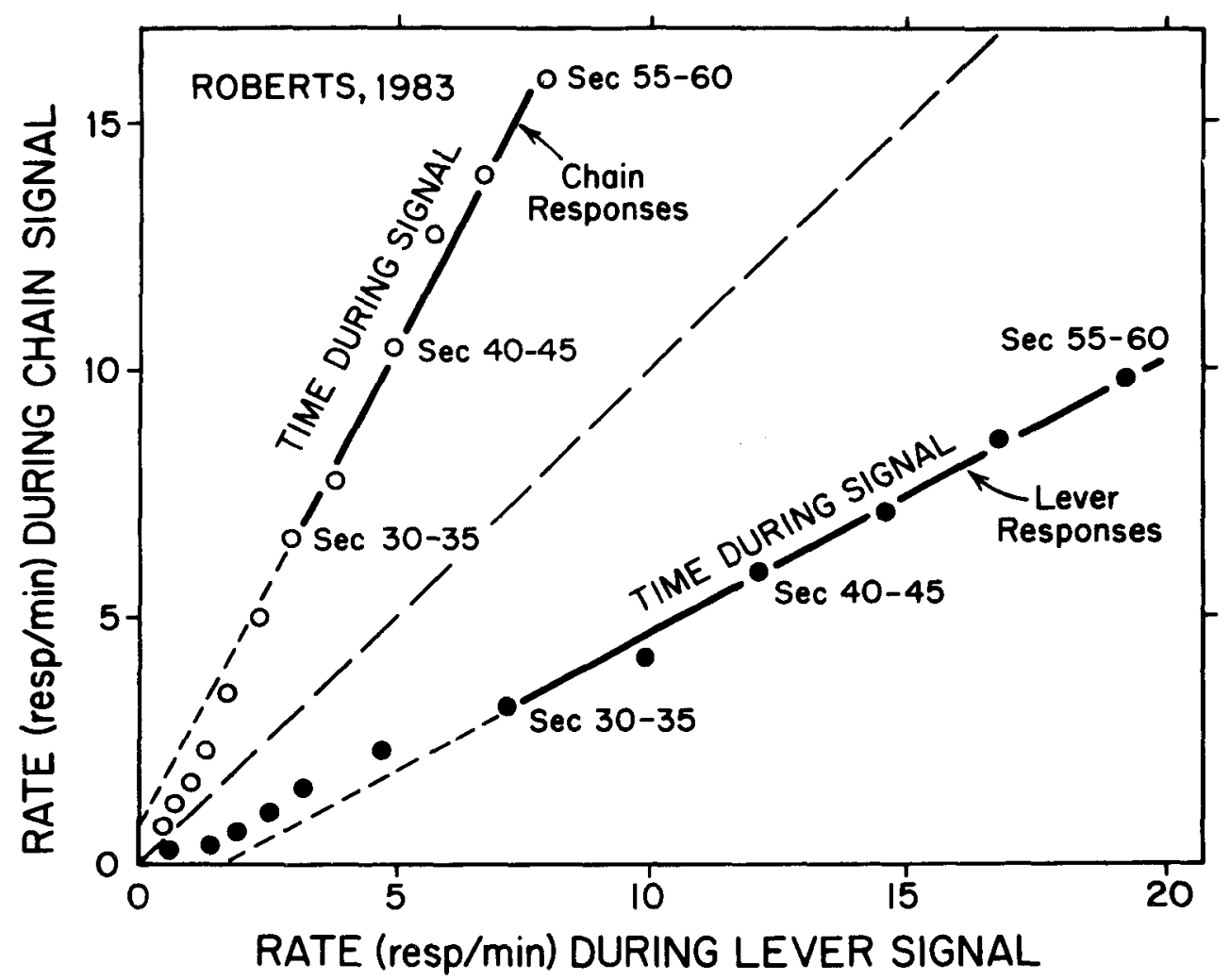

Figure 18. Time and the rewarded response had multiplicative effects during the second half of the fixed interval. Response rate during the chain signal shown as a function of response rate during the lever signal, for different parts of the fixed interval defined by the signal. Based on previously unpublished data from an experiment described by Roberts (1983). The fixed interval was 60 sec; each point represents 5 sec. The lines were fitted using only the rightmost six points of the data for each response. Each rate is a geometric mean over 6 rats. Other details as in Figure 4.

apparent generality of distinct serial processes, the new evidence provides a new reason for studying the clock-as an example of a distinct serial process. Perhaps the clock is more easily isolated than other examples (Roberts, 1981). Because the clock is sensitive to the associative value of the stimulus that it is timing (Holder \& Roberts, 1985; Roberts \& Holder, 1984, 1985), it may be quite different from the dimensional analyzers discussed earlier, which seem to be unaffected by training.

\section{Mechanisms of Learning and Motivation}

Clark's (1958) results (Figures 1, 2, and 13) suggested that learning (changed by schedule) and motivation (changed by deprivation) were due to distinct serial processes. Two other experiments support this conclusion:

1. Thomas and King (1959) used 37 pigeons to study the effect of deprivation on generalization. After the birds were trained at $80 \%$ of their ad-lib body weights, different groups were fed or starved to bring them to $60 \%$, $70 \%, 80 \%$, or $90 \%$ of their ad-lib weights. Then generalization was measured in a 90 -min extinction session. As supplementary information, Thomas and King reported response rates as a function of weight for the first quarter and the last three-quarters of the extinction session (Figure 21). Time into extinction and body weight were close to multiplicative. The intercept is not reliably different from zero $[\min t(2)=2.5]$. Skinner (1950) reported a rat example in which amount of extinction and time since feeding had close-to-multiplicative effects on leverpress rate.

2. Dinsmoor (1952) trained 6 rats to make a light/dark discrimination. Barpresses were rewarded with food pellets on a VI 2-min schedule when a houselight was on and were not rewarded when it was off. After the discrimination became asymptotic, the rats were tested at seven different levels of deprivation-at $75 \%, 80 \%, 85 \%$, $90 \%, 95 \%$, and $100 \%$ of their original body weightsand when given unlimited food. Figure 22, made by Dinsmoor, shows the results. Because the observation periods were a fixed length, the number of responses (the measure shown in Figure 22) is a fixed multiple of rate. Except for the rightmost point, the factors light/dark and level of deprivation were close to multiplicative. The line fit by Dinsmoor (Figure 22) was constrained to intersect the origin; a line fit without that constraint, not including the rightmost point, has an intercept that is not reliably different from zero $[\min t(4)=0.1]$.

Figures 7, 21, and 22-which involve the combination of hunger and reward probability-seem to provide the first convincing evidence for a theory that combines a 


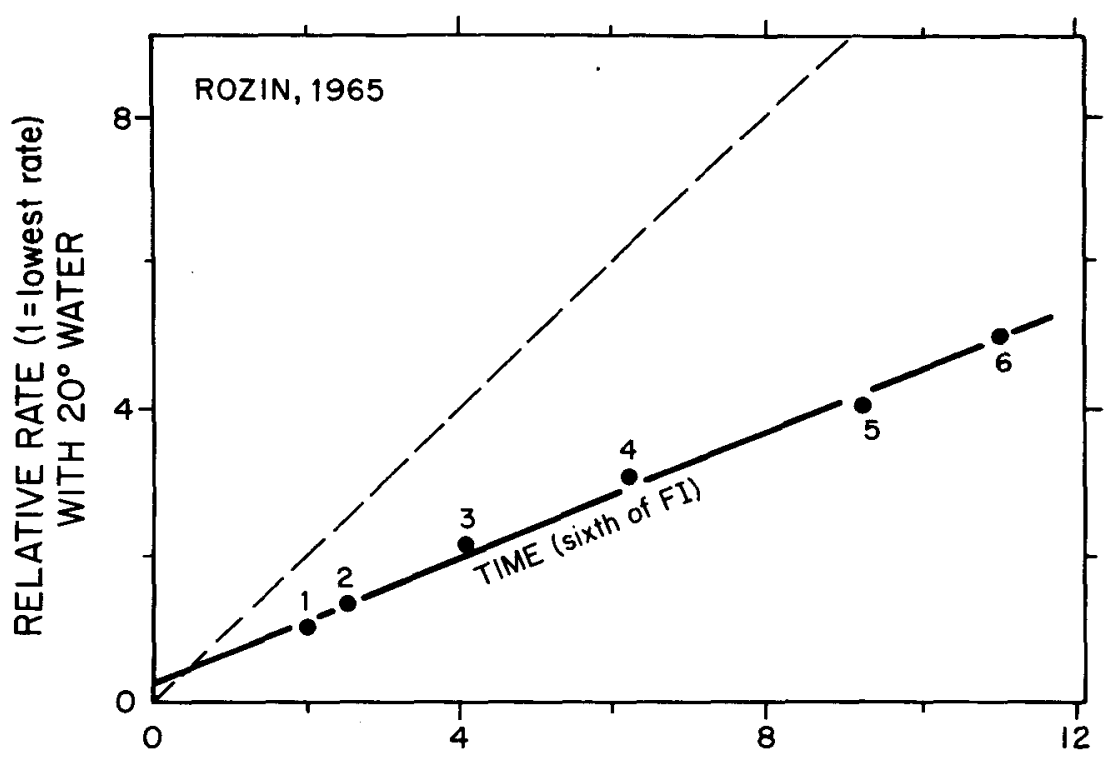

RELATIVE RATE ( $1=$ lowest rate) WITH $30^{\circ}$ WATER

Figure 19. Time and water temperature had multiplicative effects. Relative response rate with $20^{\circ} \mathrm{C}$ water shown as a function of relative response rate with $30^{\circ} \mathrm{C}$ water, for different times during the fixed interval. Based on Rozin (1965, Figure 2 and text). Rozin reports only relative rates, which were calculated for each fish at each time/temperature combination by dividing the absolute response rate at that time and temperature by the response rate of the fish at the earliest time with the colder water. Each relative rate is a geometric mean over 3 fish. Other details as in Figure 4.

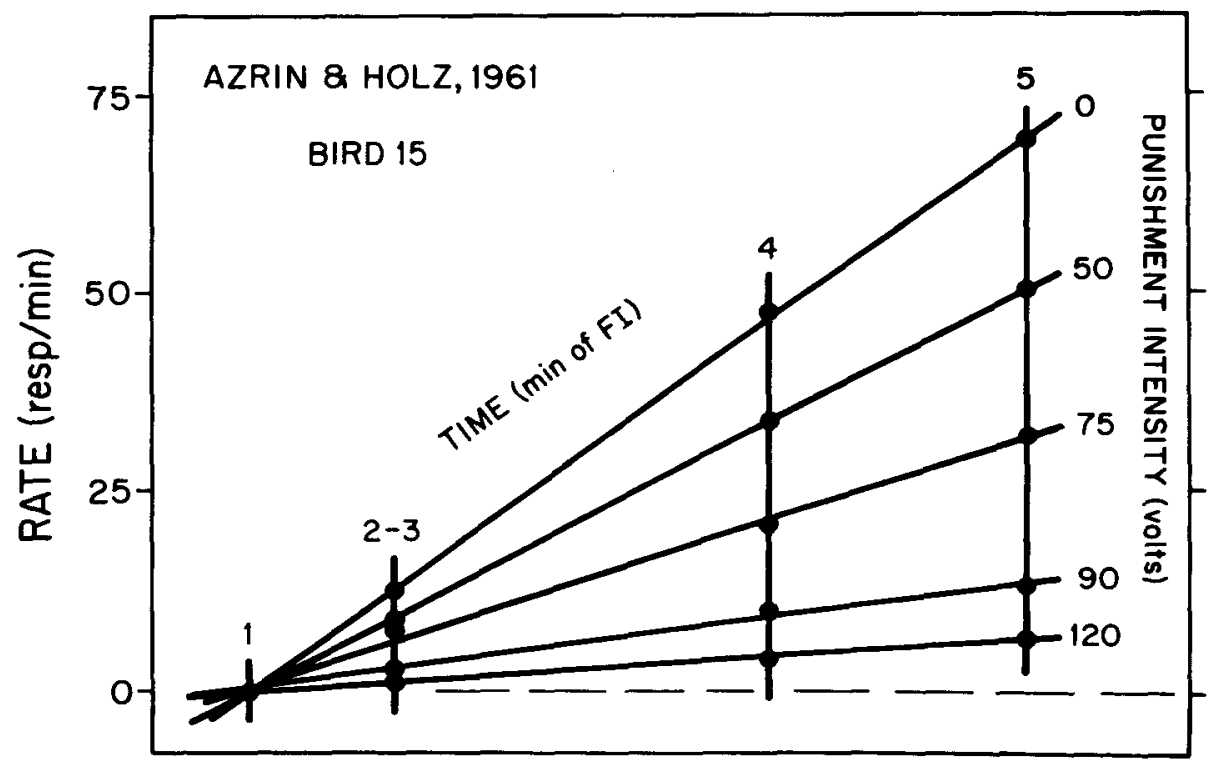

TIME EFFECT

Figure 20. Time and punishment intensity had multiplicative effects. Response rate shown as a function of the fitted minute-of-FI effect, for different minutes and punishment intensities. Based on Axrin and Holz (1961, Figure 2). The FI was 5 min. Each rate is based on "the last 5-10 days of stable performance at the designated punishment intensity." Other details as in Figure 13. 


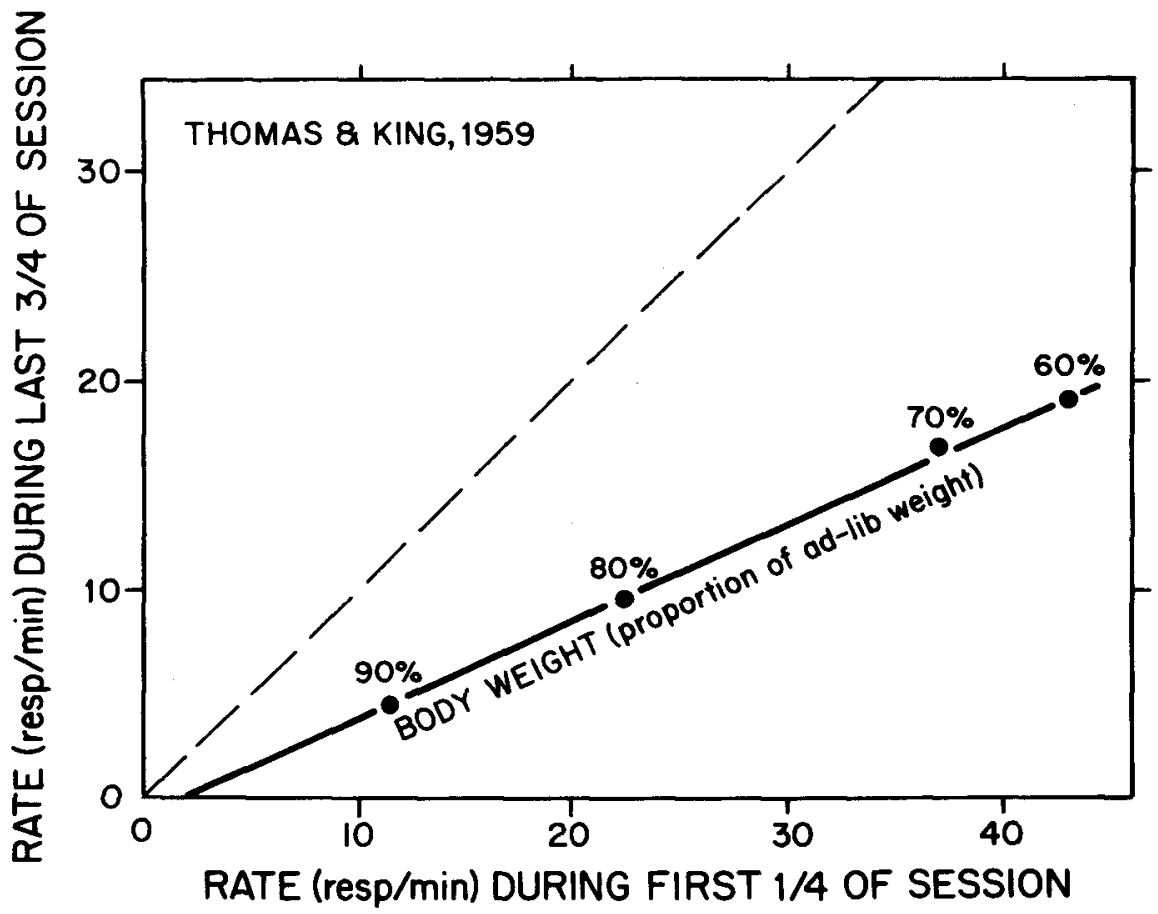

Figure 21. Amount of extinction and hunger level had multiplicative effects. Response rate late in extinction shown as a function of response rate early in extinction, for different body weights. Based on Thomas and King (1959, Figure 1). Each rate is a mean over 9-10 pigeons. Other details as in Figure 4.

statement about learning with a statement about motivation. Such theories used to be more common; Hull (1943), for example, proposed that habit strength (changed, say, by the schedule of reward) and drive (changed, say, by hours of deprivation) multiplied to produce response strength. His proposal was based on data similar to what we are considering here (Perin, 1942; Williams, 1938), but Hull's proposal differs in two ways from conclusions based on the MFM. For Hull, the multiplicative relationship between habit strength and drive was an assumptionHull assumed something resembling the data that he wanted to explain. With the MFM, the multiplicative relationship is derived from other ideas. The other difference is that Hull's theory did not specify the scale of response strength. Because factors that are multiplicative on one scale (e.g., rate) will be nonmultiplicative on nonlinear transformations of the scale (e.g., log rate), this omission made Hull's theory untestable. The MFM predicts that factors will be multiplicative on an untransformed scale of rate. Still, these examples suggest that a central part of Hull's theory, the separate determination of learning and motivation, was basically correct.

A large experiment by Miles (1956), involving 480 rats, stands a little apart from the rest of the examples of this paper. Its goal was to measure the effectiveness of secondary reinforcement during extinction. All rats were trained to press a lever for food. During training, every response was rewarded; in addition, all responses turned on a light above the food tray. The click of the pellet dispenser and the light were considered to be secondary reinforcement, although this was not tested (stimulus change can be rewarding without prior conditioning-see, e.g., Kish, 1966). Half of the rats were extinguished with both the light and the click following every response; the other half were extinguished with neither the light nor the click. The

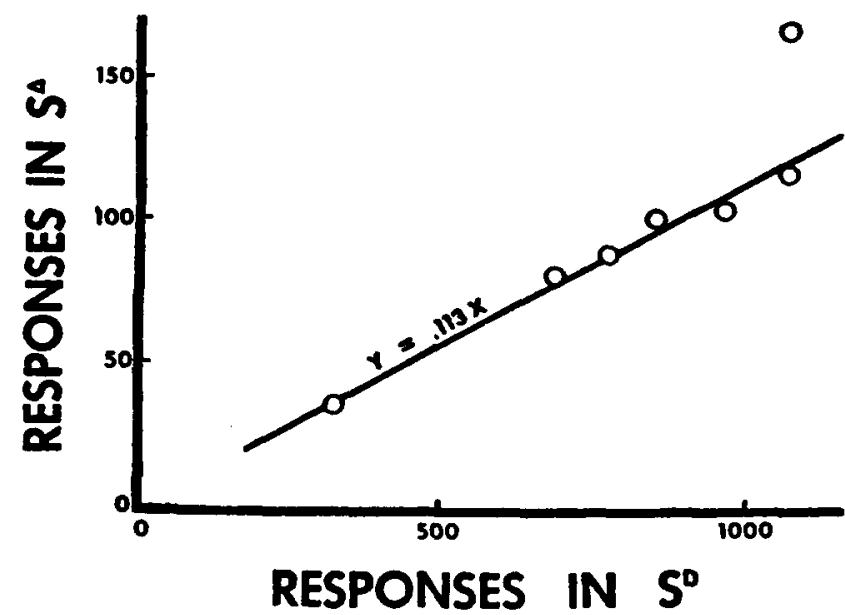

Figure 22. Reward probability and hunger level had multiplicative effects. (Tigare 2 of Dincmoor, 1952.) Totwl responses during $S^{\Delta} \boldsymbol{s}$ a function of total responses during $S^{\Delta}$ for different body weights. Foch point is based on two means over 6 rats. The rats were tested at a namber of different body weiphes, and dificerent

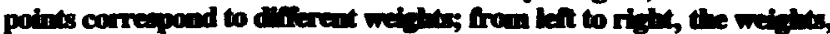
as a percentage of "normal' weifte, are: sotinted, 100, 95, 90, 85, 80,75 . The fitted lime was condrnined to pase through the origin. 
experiment had two parts. One part, using half of the rats, varied food deprivation at the time of extinction: Different rats were tested $0,2.5,5,10,20$, or $40 \mathrm{~h}$ after their last feeding. Deprivation time was factorially combined with the presence or absence of the light/click after responses. The other part of the experiment varied the amount of training ("'habit strength") before extinction: Different groups got $0,10,20,40,80$, or 160 rewards before extinction. The amount of training was factorially combined with the presence or absence of the light/click after responses.

Extinction lasted until the rat made no responses for $4 \mathrm{~min}$, and the measure of learning was the number of responses made before this criterion was met. Figure 23 shows the results-for the sake of variety, in the rateversus-part-of-fit form. The presence or absence of the light/click was close to multiplicative with both the amount of training and the amount of deprivation, and the residuals do not appear systematic. But, because the measure involved is total responses, rather than rate, the argument from data to mechanism is more complicated than usual. There are two reasons why these data suggest distinct serial processes. The first is the similarity of these data to the rest of the data considered here; it is unlikely that similar data sets will have different explanations. The second is that the data can be explained by a theory that assumes distinct serial processes if it is also assumed that the effect of time into extinction on rate was multiplica- tive with the effect of the other factors (light/click, deprivation time, and training). Because time into extinction has had multiplicative effects on rate with the factors of deprivation time (Skinner, 1950), body weight (Thomas \& King, 1959), and a factor involving training (the pigeon example from Skinner, 1950), this is a reasonable assumption.

A nice feature of the Miles experiment is that noisehere best thought of as operant level-was measured directly, by the condition with zero rewarded responses during training. Figure 23 shows that the amount of noise suggested by direct measurement was close to the amount of noise suggested by the rest of the conditions.

The deprivation-time effects in Figure 23 are interesting. Leaving aside the 0 - $\mathrm{h}$ line, the corresponding lines are spaced at close-to-equal intervals, which means that the deprivation-time effect was roughly linear with log deprivation time. In fact, the correlation between the deprivation-time effect (excluding $0 \mathrm{~h}$ ) and log deprivation time was .993 . This seems improbably high, but the Clark (1958) data (Figure 13) show the same thing: The correlation between the deprivation-time effect and log deprivation time was .994. Maybe someone else can explain this remarkable result.

If the amount of training and the presence or absence of the light/click are really changing distinct processes, this means that two response-contingent events (food and light/click) are increasing responding via different

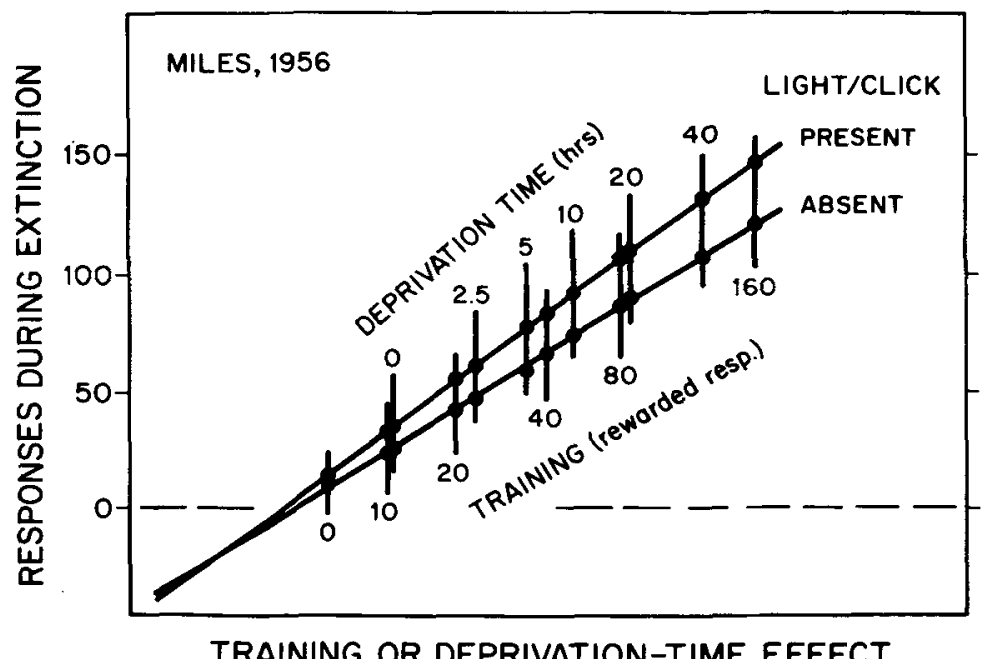

Figure 23. The effect of the presence/absence of a response marker was multiplicative with the effect of hunger level and the effect of amount of training. Responses until an extinction criterion was met shown as a function of the fitted training or deprivation-time effect, for different amounts of deprivation time and training, with a response-contingent light/click either present or absent. Based on Miles (1956, Figures 1, 3, 4, and 6). Half of the data points are from an experiment that crossed different amounts of deprivation time with the light/click present or absent; the rest are from an experiment that crossed different amounts of training with the light/click present or absent. Each number of responses is the median over $\mathbf{4 0}$ rats. Other details as in Figure 13. 


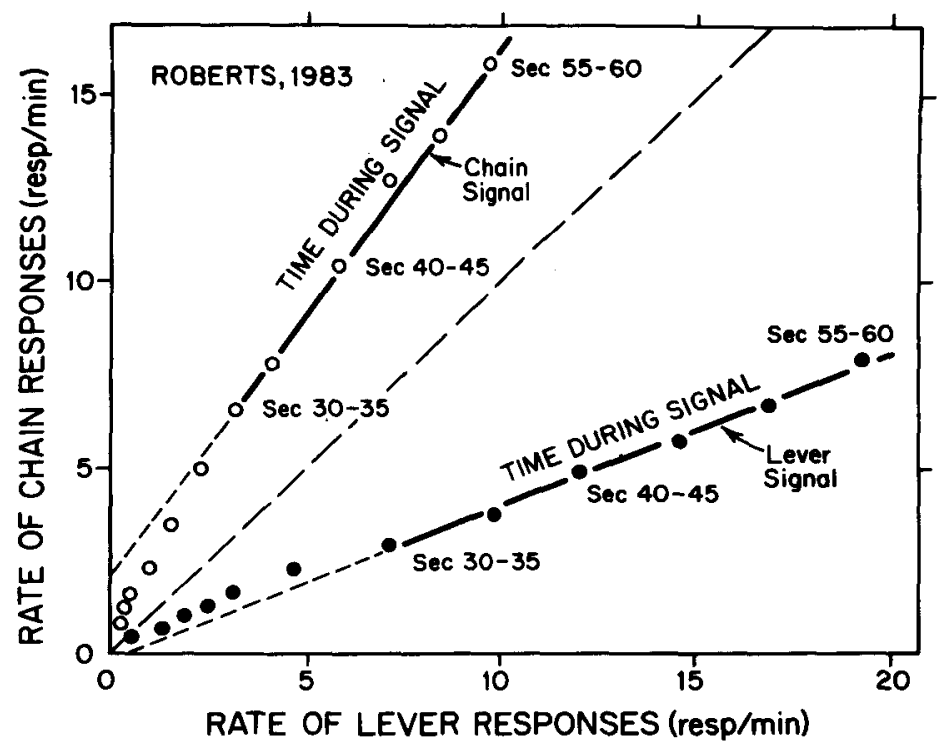

Figure 24. Time and response type had multiplicative effects during the second half of the fixed interval. Rate of chain responses shown as a function of the rate of lever responses, for different parts of the fixed interval defined by the signal. Based on previously unpublished data from an experiment described by Roberts (1983). The fixed interval was 60 sec; each point represents 5 sec. The lines were fitted using only the rightmost six points of the data for each signal. Each rate is a geometric mean over 6 rats. Other details as in Figure 4.

mechanisms. As far as I know, theories of instrumental learning have always assumed that different positive reinforcers act via the same mechanism.

\section{More Evidence for Response Elaboration}

Skinner's (1950) experiment-in which the left-key/ right-key ratio of pecks remained constant (Figure 11)provided evidence for a response-elaboration process (Figure 12) that converted a signal without a specific target into a response with a specific target (either the left key or the right key). Other experiments provide more evidence for elaboration processing (processing in which the output is more diverse than the input), and suggest that a signal can be elaborated in a number of ways.

Roberts's (1983) experiment was treated earlier as having two factors (time and signal) and two measures (chain response and lever response). It can also be treated as a three-factor experiment, with the factors time, signal, and response. Figure 18 showed that time and signal had multiplicative effects, at both levels of response; Figure 24 shows that time and response had multiplicative effects, at both levels of signal. The intercept of the chain-signal data is reliably nonzero $[t(5)=3.6, p<.05]$; the intercept of the lever-signal data is not $[t(5)=1.0]$.

The results of Figure 24 suggest that a process sensitive to time sent pulses to an elaboration process that determined whether to convert them into chain responses or lever responses. Figure 24 also suggests that the elaboration process was sensitive to the signal: During the chain signal, chain responses were more common than lever responses; during the lever signal, they were less common. Combined with the main conclusion of Figure 18 (that time and signal change distinct processes), this suggests the theory of Figure 25, where a duration-sensitive process sends pulses to a response-elaboration process that is sensitive to the modality of the signal. The ordering of processes shown in Figure 25 is based on the assumption that elaboration processes occur after other processes.

There is also evidence for response elaboration when the location of the response is constant. The most convincing data come from Blough (1978), who trained 3 pigeons with a discrete-trials wavelength discrimination procedure. Pecks to one wavelength $(582 \mathrm{~nm})$ were rewarded; pecks to other wavelengths (ranging from 575

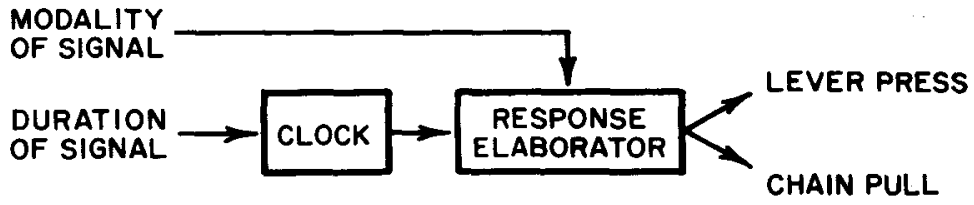

Figure 25. An explanation of the results of Figures 18 and 24 . 
to $588 \mathrm{~nm}$ ) were not. Trials were short ( $2 \mathrm{sec}$ ), and separated by a variable intertrial interval. The main measures were the latency and probability of a peck to the lighted key. Figure 26 (by Blough) shows some of the results for 1 bird. As wavelength became more different from S+, there were fewer responses in the distribution. Blough tried to indicate, via shading, the presence of two subpopulations of responses: (a) short-latency responses (unshaded), whose probability and distribution were not influenced by wavelength, and (b) longer-latency responses (shaded), whose probability was influenced by wavelength, but whose distribution was not. Figure 27, by Blough, may show the invariance of the distributions more clearly (and shows that this is a case in which the two "factors" have multiplicative effects on response probability rather than rate). It has the data for all 3 birds. Each column of points represents a different cutoff time; what is plotted are the probabilities that the bird does not peck before the cutoff time given by the label for the column (e.g., . $30 \mathrm{sec}$ ). The linearity of the data shows the invariance of the distribution shapes. Heinemann

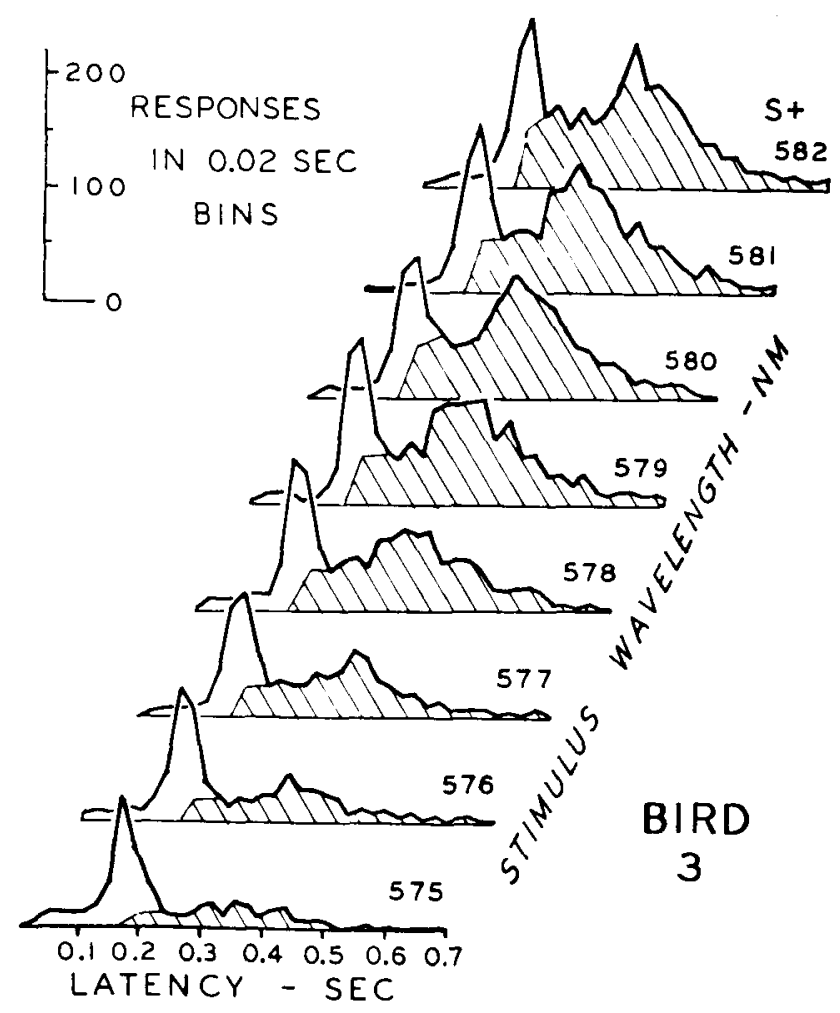

Figure 26. Frequency distributions of latencies as a function of wavelength for 1 bird. (Figure 4 of Blough, 1978.) Shading is meant to suggest the subpopulation of pecks that were controlled by wavelength; the method of determining the shading is not stated. Although test wavelengths were both more and less than the $\mathbf{S}+$ wavelength, only results with the lower wavelengths are shown. (From "Reaction Times of Pigeons on a Wavelength Discrimination Task" by D. S. Blough, 1978, Journal of the Experimental Analysis of Behavior, 30, p. 167. Copyright 1978 by the Society for the Experimental Analysis of Behavior, Inc. Reprinted by permission.)
(1974) and Mulvanny (1976) also found that the probability of a peck could change considerably without a change in the shape of the latency distribution.

In Figure 27, the existence of two subpopulations of responses-one affected by wavelength and one not-is suggested by the convergence of the lines at a single point below the upper right-hand corner. Apparently, a wavelength-sensitive process sends signals to a response elaborator, not sensitive to wavelength, that determines the latency of the response. Some responses apparently come from a second pathway, not sensitive to wavelength.

An alternative to the response-elaboration explanation is that the spread in the latency distribution is due to variation in attention to the key (S. Stemberg, personal communication, October 1985). The bird notices the key only some of the time; after it notices the key, all other processing has a fixed duration. However, this is inconsistent with the shape of the latency distribution. Suppose the probability of noticing the key is constant with time since the key becomes lit. Then the time from when the key is lit to when it is noticed will have an exponential distribution. Peck latencies, then, would be samples from an exponential distribution plus a constant (the processing time), which is much different from what was observed (Figure 26).

The response-elaboration explanation assumes that most of the variation in peck latencies was due to a single process. More support for this idea comes from the observation (Blough, 1977; Heinemann, 1974) that the latency distributions are sometimes bimodal (not including the early peak produced by fast guesses). If the durations of different processes are stochastically independent (see Sternberg, 1969a, for a possible exception), the observed bimodality must be due to the bimodality of the distribution of durations of a single process. You cannot generate bimodality by adding random variables with unimodal distributions. Moreover, the underlying process that generates the bimodal distribution must be responsible for most of the spread in the observed latencies: If a sample from a unimodal distribution is added to a sample from a bimodal distribution, the result will have a bimodal distribution only if the spread of the unimodal distribution was relatively small.

In two other cases, the data are less convincing. Corbit and Luschei (1969) measured the interlick intervals of rats (only the intervals within a burst of licking), and found that treatments with a large effect on the amount of water drunk did not change the mean interlick interval. For example, changing the duration of water deprivation from 24 to $96 \mathrm{~h}$ changed the amount drunk in a 10 -min test by $.25 \mathrm{log}$ units (from 8 to $14 \mathrm{ml}$ ), but changed the mean interlick interval by only $.006 \log$ units, not a reliable change. On the other hand, there was a reliable effect (not noticed by the authors) of deprivation time on the standard deviation of interlick intervals, about .07 log units. Changes in saccharin concentration produced similar results. The invariance of the mean interlick interval supports the idea of distinct processes controlling lick rate 

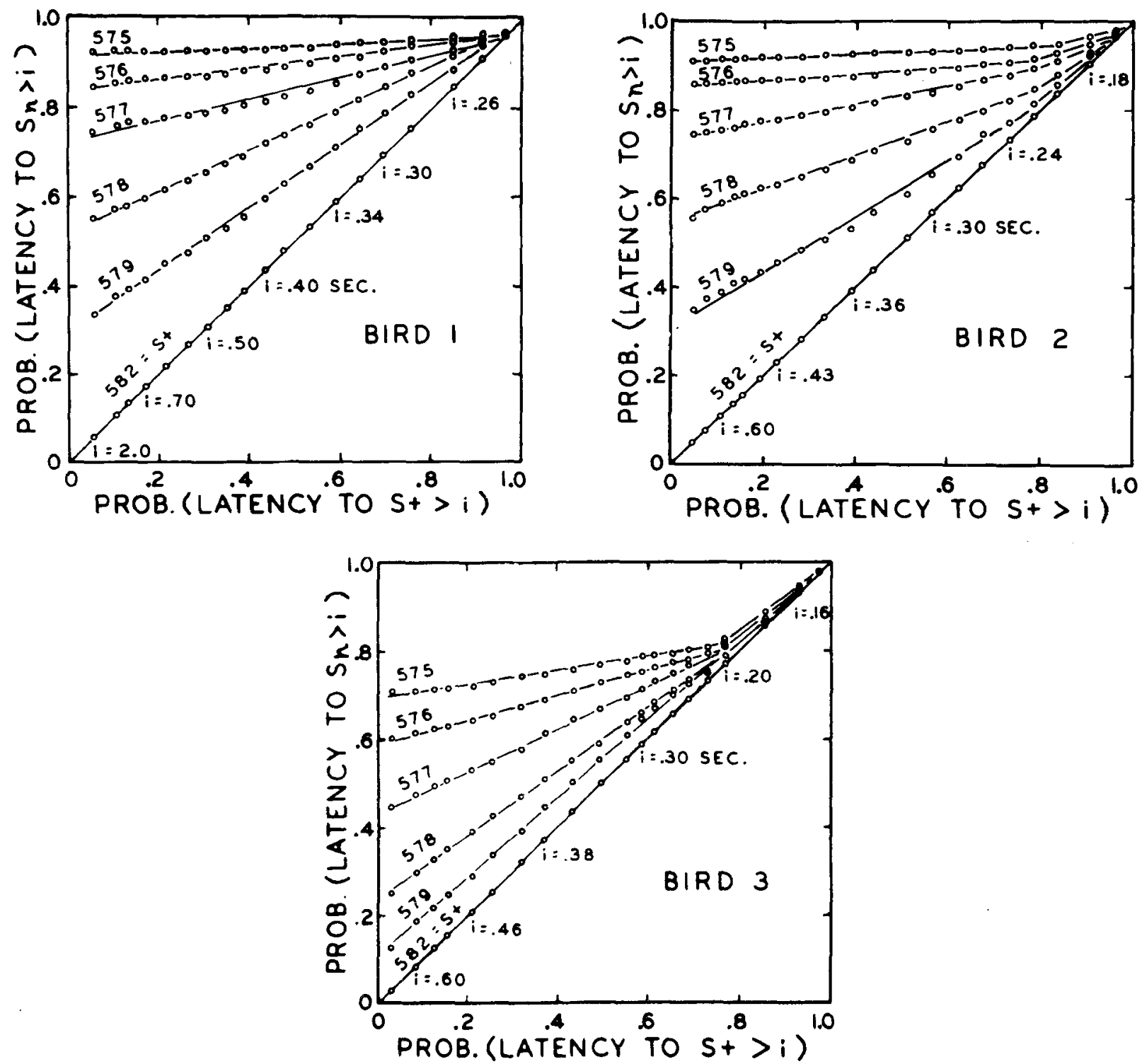

Figure 27. Probability that the peck latency to a test wavelength will be greater than a cutoff as a function of the probability that the latency to the S+ wavelength will be greater than the cutoff. Nonresponses are assumed to have infinite latencies. (Figure 2 of Blongh, 1978.) The label for each line is the wavelength in nanometers. Each vertical column of points is a different S+ latency. Although test wavelengths were both more and less than the $S+$ wavelength, only results with the lower wavelengths are shown. Lines were $n t$ by eye. Points are based on about 1,800 trials at each wavelength. (From "Reaction Times of Figeons on a Wavelength Discrimination Task" by D. S. Blough, 1978, Journal of the Experimental Analysis of Behavior, 30, p. 165. Copyright 1978 by the Society for the Experimental Analysis of Behavior, Inc. Reprinted by permission.)

and the probability of a burst of licks, but the changes in the standard deviation of interlick intervals do not. In this example, unlike any of the others, response elaboration (if credible) involves the generation of multiple responses (licks) from a single decision to respond.

The other case comes from a pigeon experiment by B. Schwartz (1977b). Schwartz found that certain kinds of punishment substantially reduced the probability of a keypeck, with only small (but reliable) effects on the duration of the peck. Schwartz gives data for 2 "representative" birds; over the five conditions of the experiment, mean peck durations varied by .07 log units (both birds) while the probability of a peck varied by $.75 \mathrm{log}$ units (one bird) or $1.12 \mathrm{log}$ units (the other bird). The difference in size between the duration effect and the probability effect at least raises the possibility that they involve different processes.

Taken together, these examples suggest that response elaboration takes place and is done by distinct processes. The range of the examples suggests that response elaboration occurs with many responses. It seems to occur with both consummatory (Blough, 1978; Skinner, 1950; and 
possibly Corbit \& Luschei, 1969, and B. Schwartz, 1977b) nonconsummatory (Roberts, 1983) responses, and the features that can be added by elaboration include location (Skinner, 1950; Roberts, 1983), form (Roberts, 1983), latency (Blough, 1978), and perhaps rate (Corbit \& Luschei, 1969) and duration (B. Schwartz, 1977b). It is sometimes under experimental control (Roberts, 1983, and probably Skinner, 1950). Because three examples (Skinner, 1950; Blough, 1978; B. Schwartz, 1977b) involve ostensibly the same response (keypeck), maybe a response is elaborated more than once.

The idea of distinct response elaboration resembles the mechanisms suggested by the evidence associated with the term command neuron. In many invertebrate experiments, stimulation of a single neuron (the command neuron) has produced a movement or a "recognizable fragment" of a movement (Bentley \& Konishi, 1978, p. 42). On the basis of these results, it is theorized that, under natural conditions, impulses from the command neuron are elaborated by later neurons to produce a relatively complex movement, such as swimming or walking (e.g., Willows, 1967; reviewed by Bentley \& Konishi, 1978, and Kupferman \& Weiss, 1978). Presumably, changes at the command neuron would change the probability of the response but not its form, whereas changes at the later neurons would change the form but not the probability. Gallistel (1980, especially chaps. 8 and 9) discusses similar arrangements, which he calls "hierarchical."

Although human sensory cortex and motor cortex are roughly equal in area (e.g., Geschwind, 1979), psychologists, with the exception of Gallistel, have had little to say about the processing that comes between the decision to respond and the response. These examples suggest a way of studying this processing. For example, treatments that change the latency of a pigeon's peck without changing the probability (in an experiment in which the probability varies considerably) can be inferred to change the response-elaboration process. Does stimulation of the motor cortex change the latency but not the probability? Does stimulation elsewhere change the probability but not the latency? Although the notion of response elaboration is probably new to most psychologists, it is easy to find everyday examples. An executive chooses an order (a "condensation" of information); his subordinates carry it out (elaboration, unless the order is remarkably detailed). A traveler chooses a destination (condensation), then decides how to go there (elaboration). A paper is written (condensation), then printed (elaboration).

The existence of response elaboration (if true) helps to answer a question raised by the rest of the examples of multiplicative factors: How can a complicated response (leverpress, keypeck), involving many muscles, be triggered by a single pulse that is processed in all-or-none fashion? What permits this simplification? The answer would be that the "complications"- the many muscle commands-are added relatively late, after the all-or-none processing of the pulse has taken place.

\section{Conclusions From the Examples Taken Together}

This section describes conclusions that are based on most or all of the examples.

1. A "theory-free" conclusion is that response rates can have more structure than is usually thought ("structure" in the sense that $n$ rates can be closely described with fewer than $n$ numbers). The usual view is that only the order of rates is likely to be meaningful or predictable. For example, Blough (1965) called rate a "conglomerate" (p. 32) and a "hodgepodge" (p. 33) and thought it unlikely that numerical predictions would be possible. Mackintosh (1974, p. 494) stated: "We do not, in general, have any independently validated way of transforming our arbitrary records of speed, amplitude, probability or rate of responding into more meaningful measures of underlying associative value, response strength, or reaction potential." This point of view can be summarized by saying that the theories (in terms of associative value, etc.) are good, but the response scales (in terms of speed, etc.) are bad. But the examples of multiplicative factors show that somewhat the opposite is true: A common response scale-response rate-reveals structure not predicted by the usual theories.

2. The method is useful. It suggested new ideas, such as the idea of response elaboration, and it shed light on preexisting ideas and questions, such as the ideas of stimulus analyzers and internal clocks-not to mention the idea of distinct serial processes. The range of topics is wide, including ideas about generalization, attention, timing, learning, motivation, and response production. It did this using preexisting experiments, showing that the necessary effort is not too great.

3. Noise is often present (assuming that nonzero intercepts indicate noise). In other words, observed responses are often produced by at least two paths, with one path insensitive to some factors that affect the other path. This is close to what Blough (1965) meant when he called rate a "conglomerate."

Moreover, the presence or absence of noise followed a pattern and made sense in terms of other knowledge. Table 2 divides 12 cases of multiplicative factors along two dimensions: (a) whether or not at least one of the two factors was a stimulus dimension (e.g., time, wavelength)

Table 2

The Presence/Absence of Noise Shows a Pattern

\begin{tabular}{|c|c|c|}
\hline \multirow{2}{*}{$\begin{array}{c}\text { Dimensional } \\
\text { Factor? }\end{array}$} & \multicolumn{2}{|c|}{ Noise Present? } \\
\hline & Yes & No \\
\hline Yes & $\begin{array}{l}\text { Blough (1978) } \\
\text { Hearst \& Koresko (1968) } \\
\text { Konick \& Thomas (1968) } \\
\text { Meltzer \& Brahlek } \\
\text { (1968, 1970) } \\
\text { Rozin (1965) } \\
\text { Tomie et al. (1973) }\end{array}$ & Azrin \& Holz (1961) \\
\hline No & Miles (1956) & $\begin{array}{l}\text { Clark (1958) } \\
\text { Dinsmoor (1952) } \\
\text { Skinner (1950) } \\
\text { Thomas \& King (1959) }\end{array}$ \\
\hline
\end{tabular}


and (b) whether or not there was evidence for noise. For the sake of independence between cases, parallel experiments (e.g., Meltzer \& Brahlek's two experiments, 1968, 1970) are treated as a single case. Experiments are classified in the "noise present" column if there was a reliably nonzero intercept on a rate-versus-rate plot or if the residuals were small relative to the distance of the intersection point from zero on a rate-versus-part-of-fit graph (Hearst \& Koresko, 1968; Konick \& Thomas, 1968; Tomie et al., 1973). The Roberts (1983) experiment is omitted because of ambiguity: One of four tests suggested noise, at $p<.05$.

Table 2 shows that the presence of a dimensional factor in the experimental design is associated with evidence for noise in the results. Taking as the null hypothesis the assumption that evidence for noise is equally likely whether or not there is a dimensional factor and treating each entry in the table as one case, the association is reliable (exact $p<.05$ ). In all cases with a dimensional factor and noise, the noise was responses not controlled by the dimension (e.g., not controlled by time). This association makes sense because dimensional control must be acquired, and it is plausible that in most cases it would not be acquired completely. The Azrin and Holz experiment is understandably different from the rest of the dimensional-control experiments because it involved much more training than the rest; the bird was given at least 2 months of training, $4 \mathrm{~h}$ per day, before data were collected. In the one nondimensional experiment that suggested noise (Miles, 1956), the noise was measured directly, and the direct measurement agreed with the estimate based on the rest of the data. Perhaps there is a small amount of operant-level noise present in most experiments, but only unusually precise experiments (such as Miles's, which used 480 rats) can detect it. The noise associated with dimensional factors is easier to detect because it consists of responses generated by reward. The results do not make sense if nonzero intercepts were due to nonindependence.

If noise, when present, was mainly responses not controlled by a dimension, this would explain why the fitting strategy of maximizing the number of degrees of freedom worked well-the dimensional factor had more levels than the other factor, and therefore was chosen as the factor that did not affect noise.

Outside evidence also suggests that free-operant responding is sometimes a mixture of responses sensitive and insensitive to certain treatments. Blough (1963), using pigeons, found that the probability of short interresponse times was unchanged by many variables that changed the probability of longer interresponse times. B. Schwartz and Williams (1972) found that long-duration keypecks were sensitive to differential reinforcement but that shortduration pecks were not; B. Schwartz (1977a) found that only long-duration pecks were sensitive to the temporal contingency provided by a differential-reinforcement-oflow-rate schedule. Roberts (1981), studying time discrimination with rats, found that the observed response-rate functions could be closely fit by assuming a mixture of responses controlled by time and responses not controlled by time.

These facts-noise (as measured by intercepts) in individual experiments shows a pattern, this pattern makes sense in terms of other knowledge, the exceptions to the pattern make sense, and there is outside evidence for noise-are important support for other conclusions from the MFM, particularly because they are considerably different from Sternberg's original evidence. The coherence and lawfulness are hard to understand if the other MFM conclusions are wrong. Noise is suggested by what remains when the multiplicative relationship is subtracted out; it would be very odd if subtraction of a meaningless amount left a meaningful amount.

4. There is wide-ranging evidence for distinct serial processes in animals. Table 1 shows that the 15 experiments involved roughly 16 different factors. The subjects were rats, pigeons, and goldfish. Over the 17 cases, the median closeness of fit, in terms of variance left, is $0.07 \%$-that is, $99.93 \%$ of the variance described. (Blough's experiment is omitted from Table 1 because of dependence between the fitted observations. Obviously, the multiplicative model fits well.) Including Blough's, the data come from 11 labs.

Although the evidence comes from only some experiments, almost all of them involved the responses (rat leverpress and pigeon keypeck) most often studied by animal psychologists. Few details of procedure were unusual. It is hard to argue that these experiments are special cases; they seem to share no procedural feature that distinguishes them from most other animal experiments. The lack of a distinguishing common feature suggests that distinct serial processes may control behavior in most or all experiments that measure leverpressing or keypecking.

5. The simplicity, precision, and generality across treatments of the data of Table 1 resemble the simplicity, precision, and generality across treatments of the AFM evidence for distinct serial processes, described in the introduction. The AFM evidence involves additive fits; the MFM evidence involves multiplicative fits with a (usually small or negligible) additive component. The AFM fits describe a median of $99.89 \%$ of the variance (median $d f=2$ ); with MFM evidence, the median is 99.93\% (3\% of the points omitted, median $d f=4$ ) or $99.89 \%$ (no points omitted, median $d f=5$ ). The AFM evidence includes 17 different treatments; the MFM evidence includes 14 .

This resemblance may be the strongest single piece of evidence that Sternberg's explanation of additive factors with RT (and the MFM explanation of multiplicative factors with rate) is correct. To illustrate the reasoning: Suppose you find a $\$ 100$ bill on the street (a rare event). Nearby, you find a $\$ 20$ bill (another rare event). Because the two bills could easily have the same source, they almost surely have the same source. It is an appeal to parsimony, made especially convincing by the rarity of the events. When brought to our attention by Sternberg (1969a, 1971), the AFM evidence had three properties 
(simplicity, precision, generality across treatments) never seen together before in behavioral data. The MFM evidence is apparently the only other behavioral data set with all three properties. Because the two data sets-two similar rare events-could easily have the same explanation, they almost surely do have the same explanation. Sternberg's explanation of the AFM data explains the MFM data with only small modifications. Other proposed explanations of the AFM data do not explain the MFM data (see below for details).

\section{CONNECTIONS WITH OTHER WORK}

\section{The Additive-Factor Method}

General ideas. The MFM was inspired, of course, by Sternberg's (1969a) AFM. The essence of the AFM is an explanation for the result that two factors have additive effects on RT. The explanation consists of four assumptions:

1. Distinct serial processes. The mechanism that generates responses can be divided into two distinct serial processes. As before, distinct means that each process can be changed without changing the other; serial means that each response requires (is preceded by) a signal from the first process to the second.

2. Processes have well-defined durations. This is equivalent to assuming that the signal from the first process to the second occurs at a definite time. The duration of the first process will be the time from the stimulus to the interprocess signal; the duration of the second process will be the time from the interprocess signal to the response.

3. Selective influence. Factor $F$ changes the first process but not the second, and Factor $G$ changes the second process but not the first.

4. Uniform signals. Changes, if any, in the information sent from the first process to the second have no effect on the duration of the second process.

On the basis of Assumptions 1 and 2, the RT is a sum, the duration of the first process plus the duration of the second. On the basis of Assumptions 3 and $4, F$ and $G$ change different elements of the sum, and thus will have additive effects on the RT.

The description given here differs in a few ways from the description given by Sternberg (1969a). One difference is that Assumptions 2 and 4 are made explicit. Assumption 2 recognizes that if the signal from the first process to the second is long-lasting (relative to the RT), it becomes meaningless, or at least arbitrary, to speak of the durations of the two processes. McClelland (1979, p. 311; see also Ashby, 1982) made clear the need for this assumption. Assumption 4 was mentioned by Pachella (1974) and Broadbent (1984a) and is implicit in comments made by Sternberg (1971). Another difference is the definition of serial. Sternberg took pains to be explicit: Two processes were serial if the second did not begin until the first had ended (Sternberg, 1969, p. 276)-a temporal definition of serial, as opposed to the information-flow definition given above. The difference, however, is minor. If two processes satisfy the temporal definition, they must also satisfy the information-flow definition. If the second process does not begin until the first has finished, it must receive some input from the first-at least a signal to begin. The temporal definition of serial essentially combines Assumptions 1 and 2; the MFM data, which are explained by a theory that includes Assumption 1 but not Assumption 2, suggest that these assumptions should be separated.

Perhaps a fifth assumption should be added. Many of the examples of multiplicative factors with rate described earlier suggested the presence of noise, responses produced by a mechanism different from the mechanism divisible into two serial processes. In the context of RT experiments, noise could be guesses, anticipations, or responses based on a different strategy. To allow for noise, yet still explain additive factors, Assumption 1 must be weakened to assume only that some responses are produced by a mechanism divisible into serial processes, and another assumption must be included:

5. Constant noise. The probability and mean RT of a response from a different mechanism are unchanged by $F$ and $G$.

Nonconstant noise is a possible explanation of cases of nonadditivity; for example, it might explain the interaction between number of alternatives and stimulus quality in a digit-naming task (Sternberg, 1969a, Experiment 5). To test this possibility, one might try to estimate the amount of noise, perhaps from features of the RT distribution (e.g., the probability of very short or very long RTs) or from error rates. With enough levels of $F$ and $G$, one can replace the constant-noise assumption with a weaker one, such as the assumption that the probability of noise is changed by $F$ but not $G$, leading to an equation in which the mean RT is a weighted sum. This parallels the MFM situation: With enough levels of $F$ and $G$, one can test models in which the straight lines do not cross the origin.

Example. A loud sound can cause a rat to make a sudden small movement, called the acoustic startle reflex. Stitt, Hoffman, and Marsh (1976) tested two ways of changing the latency of this reflex: (1) preceding the very loud (120-dB) startle-producing noise with a mild (70-dB) 1 -msec noise ending $64 \mathrm{msec}$ before the loud noise (which increases latency), and (2) preceding the loud noise with a mild 1-msec noise ending $4 \mathrm{msec}$ before the loud noise (which decreases latency). They did a factorial experiment with both types of prepulse both present and absent. Figure 28 shows the results. The points are the data; the parallel straight lines are the best-fitting additive model. The mean interaction (difference between main effect at one level and same effect at second level) was $.5 \pm .3 \mathrm{msec}$ ( $S E$ based on between-rat variability), while the main effects were 3.6 and $8.2 \mathrm{msec}$. Results for individual rats were supplied by Hoffman (personal communication, December 3,1976 ). The additive model, with $1 d f$, describes 


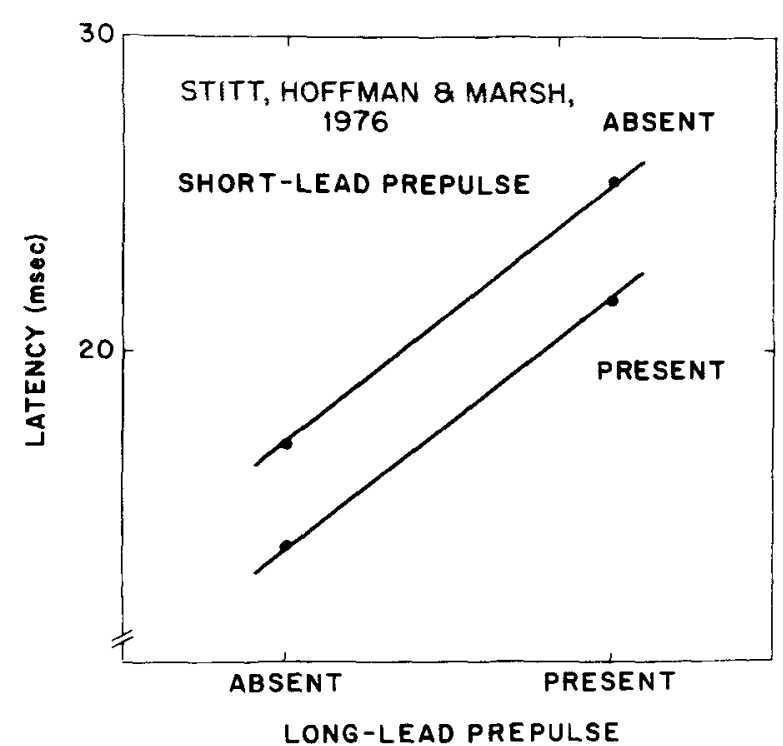

Figure 28. Startle latency as a function of the presence and absence of short- and long-lead prepulses. (Based on Hofiman, personal communication, December 3, 1976, from an experiment described in Stitt, Hoffman, $\&$ Marsh, 1976.) Each point is a mean over 5 rats, 16 trials per rat per condition. The parallel lines are the best-fitting additive model.

99.92\% of the variance of the means across rats. So the two factors were additive within experimental error, and the average error in prediction was $3 \%$ of the smaller main effect. According to the AFM, these results suggest that the startle reflex is determined by two distinct serial processes, with the short-lead prepulse changing one process and the long-lead prepulse changing the other.

An alternative explanation of the additivity is that the two prepulses changed a single process in an additive way (Broadbent, 1984a; Pieters, 1983; Sternberg, 1969a; Taylor, 1976). If so, one would expect that a third factor would either interact with both factors or add with both factors (Sternberg, 1969a). A third reliable factor in this experiment was subject $[F(4,4)=44]$. There were 5 subjects; the mean latency of the fastest rat was 3.0 msec less than the mean latency of the slowest rat. Subject interacted with presence of the long-lead prepulse $[F(4,4)=$ $7.3, p<.05]$ but not with presence of the short-lead prepulse $[F(4,4)=0.4]$, contradicting what one would expect if the two prepulses changed the same process. A model that takes these findings into account-that assumes additivity between long-lead prepulse and short-lead prepulse and between subject and short-lead prepulse, and an interaction between long-lead prepulse and subjectdescribes $99.8 \%$ of the variance $(9 d f)$ of the 20 latencies ( 4 conditions $\times 5$ rats).

These facts suggest the theory shown in Figure 29. The AFM, like the MFM, does not order the processes that it suggests; the ordering of Figure 29 (short-lead prepulse changes the earlier process) is based on the fact that the short-lead prepulse must act much sooner after its occurrence (within 10-20 msec) than the long-lead prepulse (within 70-80 msec). Because it must act quickly, its effects must be close to the periphery.

Tests of the AFM. Critisms of the AFM have included the comment that it requires acceptance of the null hypothesis (Pachella, 1974; Theios, 1973). A weakness of this comment is its lack of specificity: Any theory that predicts numbers requires acceptance of the null hypothesis. Townsend (1984) makes a more specific criticism along this line, which is too complex to discuss here. Others have emphasized the possibility that two factors may change a single process in an additive way (Broadbent, 1984a; Pieters, 1983; Taylor, 1976). Without details, this "explanation" essentially assumes what it tries to explain-no different, really, than fitting five data points with an equation that has five free parameters (see also Sternberg, 1984). Other criticisms have stated a mechanism by which two factors could change a single process in an additive way. Wickelgren $(1977,1981)$ suggested that speed-accuracy tradeoffs might produce additivity, but did not say why speed-accuracy tradeoffs should produce additivity in preference to any other result. (Of course, speed-accuracy tradeoffs would not explain the rat startle results.) Broadbent (1984b) stated that variables influencing the duration of a process fall into two classes, and only variables in one of the classes will interact if they influence the same process. But nothing was said about why this was so. Without details, this is more like an assertion than an explanation. McClelland (1979) described $a$ case where two factors are additive when one affects the rate and the other the asymptotic activation of the same process. However, this proposal implies that two measures (rate and asymptotic activation) of one process are independent; this suggests that the two measures were generated by distinct mechanisms (see "The Independent-Measures Method"' below), and that the "one" process is really two. Townsend (1984) proposed two mechanisms that produce additivity with two distinct parallel processes, but these alternatives are not plausible for any of the data described here. In both cases, the "additivity" being explained is really linearity-the task is visual search, and one factor is the presence/absence of a character at one position; the other factor is the presence/absence of a character at another position. The two factors are assumed to have exactly the same effect.

So all of the criticisms so far have conceptual problems. More important, not one of them has been supported by evidence that the AFM has led to even one wrong con-

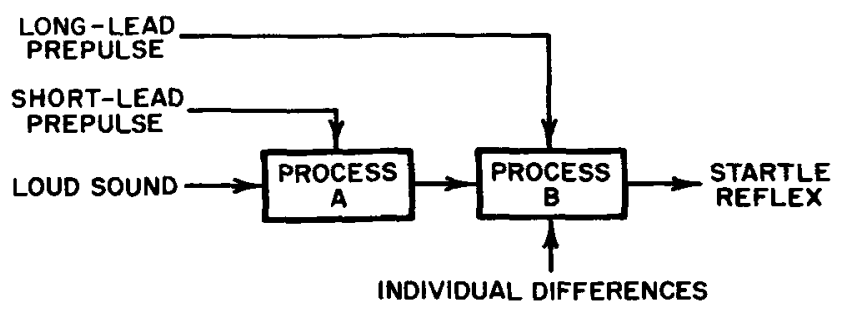

Figure 29. An explanation of the results of Figure 28, suggested by the additive-factor method. 
clusion. (The evidence given by Broadbent, 1984a, seems to be based on a misunderstanding-see Sternberg, 1984.) Still, there is considerable doubt that conclusions from the AFM are correct (Broadbent, 1984a, 1984b; Gardner, 1985, pp. 122-124; Glass et al., 1979; Grice et al., 1982; Pachella, 1974; Pieters, 1983; Taylor, 1976; Townsend, 1984; Vaughn \& Ritter, 1973; Wickelgren, 1977, 1981).

How can conclusions from the AFM be tested? There are two sources of empirical support for a theory: (1) the observations that led to the theory, and (2) other observations that the theory explains. The two kinds of support seem to be psychologically quite different: A theory based on both types of evidence is usually much more convincing than a theory based on only the first type. When it is particularly clear where to look for them, examples of the second type of evidence are often called correct predictions. (Use of the term prediction varies widely. For example, particle physicists will say that a certain theory "predicts" a certain particle when what they mean is no more specific than that evidence for the particle should be found somewhere in high-energy debris; see, e.g., Quigg \& Schwitters, 1986.) Sometimes correct predictions are more influential than other kinds of support, perhaps because they make it clearer what contradictory evidence would be.

For the AFM assumptions (distinct serial processes, etc.), the evidence in the first category (the facts that led to the theory, namely, examples of additive factors with RT) could hardly be better. To convince skeptics will require evidence from the second category. In this category, we have the MFM evidence described earlier and some examples of independent measures described below ("The Independent-Measures Method"') from animal experiments. Whatever the strengths of this added evidence, almost all of it is hard to relate to the correctness of a particular application of the AFM, the use of a specific RT result to support a specific theory. Here is an important difference between Mendel's case for genes and Sternberg's case for distinct serial processes. Mendel, after gathering and repeatedly observing the facts that led to his theory, tested his theory with backcrosses.

If we wish to test a particular application of the AFM, there are at least three possible ways:

1. Vary a third factor, in addition to the two already found to be additive (Sternberg, 1969a). Assuming that the AFM assumptions are correct, all higher order interactions (three-way, four-way, etc.) of the new factor $(H)$ with both of the two earlier factors $(F$ and $G$ ) should be nonsignificant. For instance, if the $F \times G$ interaction is nonsignificant for any fixed value of Factor $H$, the threeway interaction $F \times G \times H$ will also be nonsignificant (unless the new error term is much less). In other words, the two factors already studied should remain additive regardless of the effects of the new factor (unless the factor changes the nature of the processing; for example, practice might do this).
No experimenter seems to have done exactly this, but Sternberg (1969a) found, in three experiments, that the higher order interactions were correctly predicted. (A parallel result in the MFM data is that in Roberts's experiment, time and signal were multiplicative at two levels of response and time and response were multiplicative at two levels of signal.) A problem is that the prediction (e.g., zero three-way interaction) resembles the original result (zero two-way interaction). Mendel's backcrosses predicted a ratio (1:1) different from the ratios on which his theory was based (3:1 and 2:1).

2. Measure percent correct (Schweikert, 1985). Schweikert proposed a way of interpreting percent correct that is almost the same as the MFM. (His ideas were independent of mine.) The central idea is that in an RT experiment, two distinct serial processes will act like two filters: only some signals will be correctly transmitted, and the probability of a correct response will be the product of the probabilities of correct transmission. Thus, factors that change different processes should have multiplicative effects on percent correct, and the RT results from an experiment can be used to predict, at least roughly, the percent correct results.

Schweikert used these ideas to interpret three experiments done by others. In two of them, RT (interpreted with the AFM) and percent correct (interpreted with Schweikert's version of the MFM) led to the same conclusions. In the third experiment, there was mostly agreement, but some discrepancy; in this case, though, the AFM was applied to the whole experiment, whereas the MFM was applied separately to the two halves. One could use Schweikert's approach to examine all possible subparts of a single experiment that contained additive and nonadditive factor pairs with RT and multiplicative and nonmultiplicative factor pairs with percent correct. If the reasoning behind the AFM and the MFM is correct, there should be a correlation between deviations from additivity with RT and deviations from multiplicativity with percent correct, a prediction that does not involve accepting the null hypothesis.

3. Measure the latency of intermediate events during processing-events after the stimulus but before the response. If two events have additive effects on RT, they should act at different times during processing-another prediction that does not involve accepting the null hypothesis.

No one has deliberately tested this prediction, but the work of Ritter, Simson, Vaughan, and Macht (1982) shows the possibilities. They measured brain potentials and RTs in the same experiment. Their subjects $(n=11)$ classified visual forms. There were two factors: (a) the nature of the classification task and (b) the visual quality of the stimuli. Overall, RTs were about $560 \mathrm{msec}$. The main effects were $47 \mathrm{msec}$ (task) and $124 \mathrm{msec}$ (visual quality). The interaction was $9 \pm 11 \mathrm{msec}$, reasonably good additivity (mean $\pm S E$ based on between-subjects variance; data for individual subjects were supplied by 
W. Ritter, personal communication, August 1, 1985). Measurements of the latency of a peak found by computing the difference between two waveforms allow one to learn when the two factors were effective. The effect of task on the peak latency was $12 \mathrm{msec}, 26 \%$ of its effect on RT, whereas the visual-quality effect was $67 \mathrm{msec}$, $54 \%$ of its effect on RT. The size of the difference between these percentages suggests that, as the AFM predicts, the two factors were active at different times; however, the difference is not reliable. So this example is inconclusive. Although the percentages suggest that the two factors were active at overlapping times, this would not necessarily contradict the AFM. The AFM predicts that the two factors will act at nonoverlapping times on a single trial; the waveform data are averaged over many trials. If the latency of the transition point between processes varies from one trial to the next, factors that change different processes may have overlapping effects in averaged data.

In this example, the process changed by visual quality apparently preceded the process changed by task. If the order in which factors act can be established, one could sometimes test the AFM with a prediction of additivity. Suppose Factors $A$ and $B$ are additive and Factors $B$ and $C$ are additive. If the process changed by $A$ precedes the process changed by $B$, and the process changed by $B$ precedes the process changed by $C$, then $A$ and $C$ should be additive.

The AFM evidence for distinct serial processes. Like the MFM evidence, the AFM evidence covers a considerable range. Some of the range was described in the introduction. In the experiments cited by Sternberg (1969a, 1971), the subjects were usually young adults, but also children in kindergarten and fourth grade. The mean RTs ranged from 150 to $2,000 \mathrm{msec}$; most were between 400 and $1,000 \mathrm{msec}$. The factor effects were as large as $900 \mathrm{msec}$. One example outside this range is the rat startle example described earlier; another is provided by Rogers's (1974) experiment. In Rogers's experiment, the subject saw an item from a personality test (e.g., "I envy scholars who have the time to think"), decided whether the item described himself, and responded "true" or "false." Rogers found excellent additivity of item length and item ambiguity (the interaction was $5 \%$ of the smaller main effect) and item ambiguity and item controversiality (interaction $10 \%$ of smaller main effect). Mean RTs ranged from 3,500 to $6,200 \mathrm{msec}$. The range and precision of the AFM evidence for serial processes make the range and precision of the MFM evidence more credible. Because the AFM evidence more or less covers the whole range of RT experiments, it is plausible that the mechanism responsible will be important outside that range, such as with the MFM procedures.

Just as time into FI is multiplicative with a number of other factors in response-rate experiments, certain factors are often additive with other factors in RT experiments. In an item-recognition procedure, list length is additive with speed-accuracy instructions, preview, input hemisphere, stimulus quality, stimulus redundancy, response type, response frequency, practice, age, and the difference between alcoholics and schizophrenics, to mention only the examples given by Sternberg $(1971,1975)$. These findings suggest the existence of a process changed by list length and not by many other factors, sometimes called a memory-search stage. In many RT procedures, stimulus "quality"-amount of contrast or visual noise for visual stimuli, amount of background noise for auditory stimuli-is usually additive with other factors. It is additive with list length (Bracey, 1969; Hardzinski \& Pachella, 1980; Sternberg, 1967; Tharp, Rundell, Lester, \& Williams, 1974; and Ford \& Banks, 1977, where the quality factor was handwritten versus typed; see Hardzinski \& Pachella, 1980, for an exception), visual-array size (Logan, 1978), stimulus-response compatibility (Biederman \& Kaplan, 1970; Blackman, 1975; Frowein \& Sanders, 1978; Shwartz, Pomerantz, \& Egeth, 1977; Sternberg, 1969a), pattern goodness (Checkosky \& Whitlock, 1973), foreperiod length (Bernstein, Chu, \& Briggs, 1973), stimulus similarity (Shwartz et al., 1977), an irrelevant auditory cue (Acosta \& Simon, 1976), stimulus probability (Miller \& Pachella, 1973; Pachella \& Miller, 1976; Stanovich \& Pachella, 1977; see Stanovich \& Pachella, 1977, for some exceptions); stimulus contrast (Sanders, 1980); and word frequency (Stanners, Jastrzembski, \& Westbrook, 1975; Landauer, Didner, \& Fowlkes, 1975). These findings suggest the existence of a process changed by stimulus quality and not by many other factors, often called an encoding stage. When a factor is additive with many other factors, it becomes harder to believe that single examples of additivity-at least, with that factor-are due to some sort of coincidence, such as two factors that change a single process in an additive way (Broadbent, 1984a; Pieters, 1983; Taylor, 1976) or speedaccuracy tradeoffs that produce additivity (Wickelgren, 1977, 1981). For a more extensive review of the AFM evidence, see Sanders (1980).

Comparison with the MFM. The AFM and the MFM explain different data in almost the same way. Of the five AFM assumptions, four are identical or almost identical to MFM assumptions. Assumption 2 (processes have well-defined durations) does not obviously resemble its MFM counterpart (signals are countable), but the two assumptions are essentially the same. Both of them require that the signal (transfer of information) from the first process to the second happen at a definite time (definite relative to the total time involved-either the total RT or the average time between signals). Without a definite time, one cannot say when the first process ends (or the second begins), nor can one increment one's count. Both assumptions are most clearly met when signals from the first process to the second consist of pulses or, at least, when they have a sharp leading or trailing edge. In an RT experiment, this assumption is made more plausible by the fact that the stimuli have quick onsets. In a response-rate experiment, there is no corresponding procedural detail, making the observation of multiplicative factors with rate 
in a way more telling than the observation of additive factors with RT.

What does the MFM evidence add? Given the AFM evidence for distinct serial processes, what does the MFM evidence add?

First, it strengthens the case in four ways. From strongest to weakest:

1. As mentioned earlier, the AFM evidence and the MFM evidence share a feature not found in any other behavioral data: A simple fit closely describes the effect of a wide range of treatments. Because the two data sets could easily have the same explanation, they almost surely $d o$ have the same explanation. Without this similarity, there is much less reason to believe that the two data sets had the same explanation, and a critic of the explanation given here could offer two alternative explanations: one for the AFM data, another for the MFM data. With this similarity, an alternative explanation, to be plausible, must explain both data sets-a much harder task.

2. As mentioned earlier, conclusions about noise from the MFM evidence have internal regularity and make sense in terms of other knowledge. The conclusions about noise are a byproduct of the main assumptions (e.g. , the assumption of distinct serial processes); they explain why the fitted lines do not cross at zero. If the main assumptions are wrong, it is hard to see why the conclusions about noise are so understandable-subtraction of something meaningless should not leave something meaningful.

This argument supports the existence of distinct serial processes without assuming that simple close fits to data are unlikely to be fortuitous, an assumption needed by most of the rest of the evidence. Perhaps this assumption is questioned by the critics of the AFM who propose that two processes can change a single stage in an additive way (Broadbent, 1984a; Pieters, 1983; Taylor, 1976) or that additivity can be due to speed-accuracy tradeoffs (Wickelgren, 1977, 1981). Another important feature of this argument is that it is based on data (the size of intercepts) that is quite different from the original data (additivity with RT).

3. Alternative explanations of the AFM evidence do not explain the MFM evidence. If two factors change a single process in an additive way (Broadbent, 1984a; Pieters, 1983; Taylor, 1976), this will not produce multiplicative factors. Given the procedures involved, there is no reason for speed-accuracy tradeoffs (Wickelgren, 1977, 1981) to be important. The alternatives given by Townsend (1984) assume that the two factors have exactly the same effect on the dependent measure, which is not true for any of the MFM examples.

McClelland's (1979) proposal-two factors are additive because one changes the rate and the other the asymptotic activation of the same process-can be modified to predict multiplicative factors with rate by assuming that one factor affects whether or not the process makes any response and the other process affects the amplitude of the response. If a later process filters signals according to their amplitude, this could produce multiplicative ef- fects. The problem here is essentially the same as the problem with McClelland's explanation of additivity: The proposal implies that two measures of one process are independent, suggesting that the "one" process is really two (see "The Independent-Measures Method" below). The response-elaboration evidence makes it plausible that response amplitude and probability could be determined by distinct serial processes.

4. An observation of additive factors suggests the existence of distinct serial processes only if it is assumed that other mechanisms are unlikely to produce additive factors with RT. But, as mentioned earlier, this assumption has been questioned. The MFM evidence supports the existence of distinct serial processes via a parallel, but different, assumption: Other mechanisms are unlikely to produce multiplicative factors with rate.

Second, it increases the generality of the theory, that is, the range of data that it explains. Most of the increase in generality is procedural. The AFM evidence for distinct serial processes, with the exception of the rat startle example, comes from experiments that share many features: human subjects, responses that are triggered by an external signal, responses that require little movement, treatments that have only a slight effect on the probability of a response, an emphasis on speed, an emphasis on accuracy, verbal instructions, secondary reinforcement (such as money) for performance, and low levels of practice (a few hours or less), to name a few. Perhaps results from signal-detection experiments are part of the preMFM evidence for distinct serial processes (e.g., Swets, 1986), but they would increase the generality of the earlier evidence only slightly. With the possible exception of Blough (1967), there seem to be no convincing animal data supporting signal-detection theory.

Given only the AFM evidence, there is no clear reason, except for the rat startle example, to think that distinct serial processes control behavior in other situations, that is, experiments without these features. The MFM experiments, however, have none of these features. Maybe the only features shared by the AFM evidence and the MFM evidence are the use of vertebrate subjects and the measurement of brief responses (buttonpress, leverpress, spoken word, keypeck), actions that are completed in less than a second or so. The restriction to brief responses is not trivial, of course; some well-studied actions last longer: solving a maze, traversing a runway, speaking a sentence. Still, the near-zero overlap between the AFM procedures and the MFM procedures suggests that distinct serial processes may be important in a very wide range of experiments. It also makes it hard to specify the range. If a new species of ant is found in both Iowa and California, it probably exists in many states, but it is hard to say which states.

The increase in generality also involves the range of theories in which distinct serial processes play a part. The MFM evidence, unlike the AFM evidence, suggests that distinct serial processes sometimes control behavior within a more elaborate mechanism. In a number of cases, there 
was evidence for distinct serial processes and evidence for a parallel path that produced noise. The AFM has not suggested such arrangements. In a few cases (Hearst \& Koresko, 1968; Tomie et al., 1973), the parallel path apparently produced a substantial fraction of the total responses. In these cases, to describe the whole mechanism as "serial" would be a nontrivial distortion. In addition, the MFM evidence, unlike the AFM evidence, suggests that distinct serial processes can be involved in response elaboration, a fundamentally different kind of processing.

Although this paper has emphasized the assumption of distinct serial processes, findings of additive factors with RT and multiplicative factors with rate also support the rest of the assumptions. Given the multiple connections of most areas of the brain, Assumption 3 (selective influence) may be unexpected. Assumption 2 (sharp leading edge) and Assumption 4 (uniform signals), if true, reveal something about the nature of the communication between the processes that generate responses. (It is interesting that all-or-none action potentials have the necessary features: they rise quickly, and they have the same shape and height regardless of how they are produced. Of course, two similarities are not proof of identity.) In a wide range of experiments, responses seem to be generated by signals that are relatively uniform and have a sharp leading edge.

The MFM evidence suggests that the AFM evidence did not get the attention it deserved. Historians of science sometimes say that this or that work was initially neglected because "the time was not ripe." Soon after Sternberg proposed the AFM, in the late $60 \mathrm{~s}$, at least one person told him that a serial theory of processing was "obvious", (S. Sternberg, personal communication, April 5, 1986), and, at the time, serial theories were already popular (e.g., Smith, 1968). In the case of the AFM, maybe the time was overripe.

\section{The Independent-Measures Method}

General ideas. Most experimental psychologists are familiar with the term double dissociation, introduced by Teuber (1955) to describe a pattern of lesion results: lesions in Place $A$ (e.g. , inferotemporal cortex) impair performance on Task $X$ (e.g., object learning) but not Task $Y$ (e.g., size discrimination), whereas lesions in Place $B$ (e.g., striate cortex) impair performance on Task $Y$ but not Task $X$. The conclusion from this pattern of results is that the two places perform different functions (e.g., Kirn \& Floody, 1985; Shankweiler, 1966; W. A. Wilson \& Mishkin, 1959). Since then, the concept of double dissociation has widened to include any treatment, not just lesions, and examples of double dissociation have been used to draw conclusions not only about localization but also about the existence of distinct mechanisms. Satinoff (1978), for example, used lesion results to conclude that thermoregulation in rats was accomplished by more than one thermostat; Blakemore and Campbell (1969) used a selective-adaptation procedure to conclude that there were distinct size-sensitive channels in the human visual sys- tem; and Posner, Lewis, and Conrad (1972) used RT experiments to suggest that name matching and visual matching involved distinct mechanisms. An example from animal cognition is found in Maki's (1979) studies of pigeon matching-to-sample, which showed that a measure of associative learning and a measure of short-term memory could be changed independently, suggesting distinct memory systems.

We deal here with a similar, but more specific, result called independent measures (Roberts, 1981, 1983): the finding that two measures taken at the same time can be changed independently. In contrast, double dissociations almost always involve two measures taken at different times. For example, in Maki's (1979) work, one measure came from responses to the first event in a three-event sequence (keylights-food/no food-keylights) and the other measure came from responses to the last event. Another difference is that most cases of independent measures involve two qualitatively different measures-for example, latency and amplitude, or sensitivity and bias; most cases of double dissociations involve two similar measuresfor example, percent correct with two different tasks or RT with two different tasks. The differences are important because the two types of results-independent measures and most double dissociations-suggest different conclusions. Most double dissociations suggest that a process that controls one response is distinct from a process that controls another response; a case of independent measures suggests that a single response is controlled by distinct processes.

The conclusion that a single response is controlled by distinct processes resembles the conclusions from the AFM and the MFM. To stress similarities with the AFM, Roberts (1983) called this pattern of reasoning the independent-measures method (IMM). To compare the different methods, it will help to make the assumptions of the IMM explicit:

1. Distinct processes. The mechanism that generates responses can be divided into two distinct processes, $A$ and $B$.

2. Selective influence. Factor $F$ changes $A$ but not $B$; Factor $G$ changes $B$ but not $A$.

3. Selective measurement. Measure $X$ is sensitive to changes in $A$ but not $B$; Measure $Y$ is sensitive to changes in $B$ but not $A$.

If the assumptions are true, Factor $F$ should change Measure $X$ but not Measure $Y$, and Factor $G$ should change $Y$ but not $X$-a case of independent measures.

Examples. Independent measures are much less common than double dissociations. One animal example, maybe the clearest, comes from an experiment with the peak procedure (Roberts, 1981), a time-discrimination procedure that resembles a discrete-trial fixed-interval schedule. On some trials, rats received food for the first response made more than a fixed duration (e.g., $40 \mathrm{sec}$ ) after a signal (light or sound) had begun; on other trials, the signal lasted a long time (e.g., $120 \mathrm{sec}$ ), and no food 
was given. After training with this procedure, response rate reached a maximum during the signal at about the time that food was given. Figure 30 shows the effect of changing the time of food (upper panel) and the probability of food (lower panel). A change in the time of food changed peak time (the time of the maximum) but not peak rate (the value of the maximum), whereas a change in the probability of food changed peak rate but not peak time. For peak time, the probability-of-food effect was $6 \%$ of the time-of-food effect; for peak rate, the time-of-food effect was $1 \%$ of the probability-of-food effect. There are a number of other treatments that change peak time and peak rate selectively (Roberts, 1981; Roberts \& Holder, 1984). These results suggest that responses were controlled by two distinct processes, one sensitive to the time of food and the other sensitive to the probability of food.

Another example involves the rat acoustic startle reflex. The work of Hoffman and his colleagues has shown that the amplitude and latency of the reflex can be changed independently. Long-lead sensory changes, both visual and auditory, reduced the amplitude, but short-lead changes left amplitude unchanged or increased amplitude (Hoffman \& Searle, 1965; Hoffman \& Wible, 1969, 1970; Marsh, Hoffman, Stitt, \& G. M. Schwartz, 1975; G. M. Schwartz et al., 1976; Stitt et al., 1976; Stitt, Hoffman, Marsh, \& Boskoff, 1974). Long-lead sensory changes increased latency or left it unchanged; short-lead changes reduced latency (Hoffman \& Wible, 1970; G. M. Schwartz et al., 1976; Stitt, Hoffman, \& Marsh, 1973). In a single
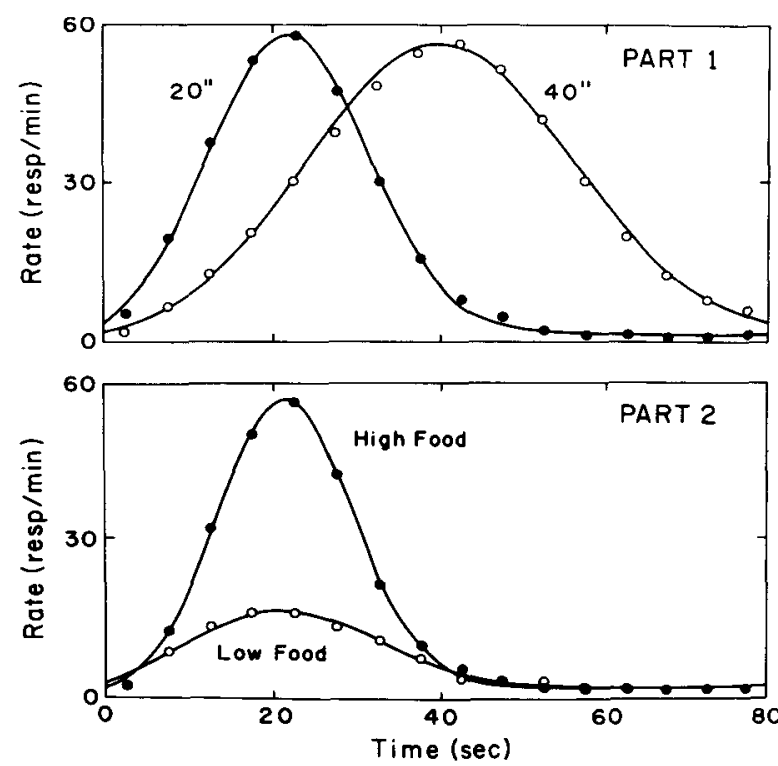

Figure 30. An example of independent measures: Response rate as a function of time into the trial. (Figure 2 of Roberts, 1981.) During Part 1 of the experiment, the two response-rate functions differed in peak time but not peak rate; during Part 2, the two functions differed in peak rate but not peak time. Each point is a mean over 10 rats. (From "Isolation of an Internal Clock" by Seth Roberts, 1981, Journal of Experimental Psychology: Animal Behavior Processes, 7. Copyright 1981 by the American Psychological Association. Reprinted by permission.) experiment, Stitt et al. (1974) found that the presence of continuous noise increased amplitude and did not change latency, whereas noise started $3 \mathrm{msec}$ before the startleeliciting sound decreased latency and did not change amplitude. In addition, the thresholds for the short-lead effect (on latency) and the long-lead effect (on amplitude) were much different: the long-lead changes could be produced by stimuli about 100 times weaker (G. M. Schwartz et al., 1976). These results lend considerable support to the AFM conclusion that a short-lead prepulse and a longlead prepulse change distinct processes. This may be the most convincing validation so far of a particular application of the AFM.

There are only a few other examples of independent measures with animals. Roberts (1982) found that the relative spread of the functions generated by the peak procedure (e.g., Figure 30) could be changed without changing peak time and peak rate, and that peak time and peak rate could be changed without changing relative spread. Roberts (1983), in a different analysis of an experiment considered earlier (Figures 18 and 24), found that relative response rate (local response rate divided by overall response rate) and the proportion of chain responses could be changed independently. Roberts and Holder (1985), measuring time discrimination with a psychophysical choice procedure, found that a measure of sensitivity (accuracy with the short signal) and a measure of bias (accuracy with the short signal minus accuracy with the long signal) could be changed independently. Oddly enough, there seem to be no examples with animals in which the usual measures of sensitivity and bias are independent (Blough \& Blough, 1977). Edmonds and Gallistel (1974, Figure 9) report two measures of the effect of electrical brain stimulation that might be independent. The only human examples that I know of come from Murdock (1974, pp. 34-35) and Sternberg, Wright, Knoll, and Monsell (1980).

Comparison with the MFM. Because one can get evidence for distinct processes (via the IMM) that may or may not be serial, this paper uses the term distinct serial process, separating the two properties, rather than processing stage, the term used by Sternberg (1969a) and many others to mean more or less the same thing.

The MFM makes five assumptions that the IMM does not (serial arrangement, countable signals, uniform signals, constant noise, independence), which might suggest that the IMM will be more widely usable; but the IMM makes one assumption that the MFM does not (selective measurement), which might suggest the opposite. So far, there are many more cases of multiplicative factors than independent measures, at least with animals.

Compared with the AFM and the MFM, the IMM seems to be better for determining the nature (as opposed to the existence) of a process. This is because a result of independent measures supports Assumption 3, the assumption of selective measurement. The results of Figure 30, for example, suggest that peak time is a selective measure of some of the mechanisms involved in time dis- 
crimination. Many experiments (e.g., Holder \& Roberts, 1985; Maricq, Roberts, \& Church, 1981; Roberts, 1981, 1982; Roberts \& Holder, 1984) have been based on this conclusion. The reasoning is simple: If a factor changes peak time, it changes these mechanisms; if it does not change peak time, it does not change these mechanisms (see Roberts, 1981, for details). In other words, a onefactor experiment will tell you if the factor changes the peak-time mechanism. Cases of additive factors with RT have been used as the basis for further work (e.g., Duncan \& McFarland, 1980; Egeth, 1977, pp. 310-316; Logan, 1978; Stanovich \& Pachella, 1977; Wishner, Stein, \& Peastrel, 1978), but there are difficulties. Suppose you find that Factor $F$ (e.g., visual noise) is additive with other factors, and you want to learn about the process that is sensitive to visual noise, call it Process $X$. To learn if Factor $G$ influences Process $X$, you must do a two-factor experiment, with $F$ and $G$. And if $F$ and $G$ interact, you face a problem of interpretation: Did $G$ change Process $X$, or did it change the signal sent to Process $X$ (e.g., Sternberg, 1969b, Sections 6 and 7)?

\section{Quantitative Theories as Tools}

There seems to be a consensus that quantitative theories (theories that predict numbers) are better than nonquantitative ones (e.g., Commons, 1981), but quantitative theories of animal learning, beginning with Hull and continuing through the work of Bush, Estes, Mosteller, and others in the 50s (reviewed by Sternberg, 1963), have not fared well. A classical idea about numerical computation is "the purpose of computing is insight, not numbers" (Hamming, 1962, p. v). This applies to theories, too, and it would be hard to say that more insight came out of quantitative learning theories than went into them. For example, they did not lead to important nonquantitative ideas, they did not reveal connections with other lines of work, and they did not suggest new ways of doing experiments. They receive little attention in current animal-learning texts (e.g., Flaherty, 1985). In contrast, signal detection theory, developed at roughly the same time, did lead to important nonquantitative ideas (e.g., an alternative to the idea of a threshold) and new ways of doing experiments.

What was wrong? Perhaps quantitative learning theories really did aim at providing numbers-close fits-rather than insight, and perhaps they did so because their creators saw no other reasonable goal. Even today, most quantitative theories of animal behavior seem to be justified mainly by the fact that they provide close fits to data. The idea that a quantitative theory can be a tool-can lead to nonquantitative conclusions, conclusions in which there is no trace of the quantitative reasoning used to reach them-may be unfamiliar to most animal psychologists, perhaps because there are no well-known examples in the area.

There are many examples in other areas. Examples from human cognitive psychology, besides the AFM, include Shaw's (1980) use of inequalities of probabilities to determine if an increase in the number of locations watched lowers stimulus quality (its internal representa- tion) and Meyer, Yantis, Osman, and Smith's (1985) use of mixtures of distributions to choose between discrete and continuous models of response preparation. In neurophysiology, a simple theory about synaptic function, called quantal analysis, involving the Poisson distribution, has been used to determine whether the changes that produce habituation are pre- or postsynaptic (Castellucci \& Kandel, 1974). Some examples from genetics are Mendel's prediction of seed ratios, leading to the postulation of genes, which was based on the multiplication of probabilities; Sturtevant's construction of linear gene maps, also based on the multiplication of probabilities (Sturtevant \& Beadle, 1939/1962); and Luria and Delbruck's use of the Poisson distribution to conclude that phage-resistant bacteria arise before contact with phage (Ayala \& Kiger, 1984 , p. 680). Thus, the MFM is one example of a widespread kind of theorizing - the use of a quantitative theory as a tool-that seems to have been neglected in the study of animal behavior.

\section{SUMMARY}

The MFM is not as novel as it might appear to a specialist in animal behavior. It is part of a family of methods - a family that includes the AFM and the IMMrelated in the sense that they lead to similar conclusions in similar ways. Perhaps the MFM helps to show that such a family exists. To some extent, each method in the family can test conclusions based on the other methods, a kind of cross-validation exemplified by the rat startle example (involving the AFM and the IMM) and by Schweikert's (1985) work (involving the MFM and the AFM). More broadly, the MFM is one of many examples of the use of a quantitative theory as a tool.

The idea of distinct serial processes, used by Sternberg $(1969 a, 1971)$ to explain one set of data-additive factors observed in human RT experiments-also explains a new set of data-additive factors with startle latency, independent measures, and, of course, multiplicative factors with response rate and probability-collected under much different conditions. The new data seem to make the case for Sternberg's explanation much stronger. Maybe the most convincing point is that the old data set and part of the new data set (the MFM evidence) share a feature apparently found in no other behavioral data: In both cases, a simple fit closely describes the effect of a wide range of factors. Because the two data sets could have the same explanation-Sternberg's-they almost surely do have the same explanation. If they had different explanations, it would be like lightning striking twice in the same place for different reasons. Furthermore, conclusions about noise based on the MFM data make sense in a number of ways that are hard to explain if Sternberg's explanation is wrong, and the new data support Sternberg's explanation without making assumptions that the AFM evidence required.

The new evidence seems to greatly increase the generality of distinct serial processes, the range of situations 
Table 3

Summary of Animal Evidence for Distinct Serial Processes

\begin{tabular}{cccc}
\hline Type & Examples & Factors & Labs \\
\hline Multiplicative factors & & & \\
with rate or probability & 18 & 14 & 11 \\
Additive factors with RT & 1 & 2 & 1 \\
Independent measures & 5 & 13 & 2 \\
\hline
\end{tabular}

in which they control behavior. Table 3 summarizes the animal evidence for distinct serial processes. It shows that there are many examples, involving many factors, from many labs. The earlier evidence, with perhaps a few exceptions, was limited to human RT experiments; the only procedural features shared by the new (animal) evidence and the older (human) evidence seem to be the use of vertebrate subjects and the measurement of brief responses. The near-zero overlap suggests that distinct serial processes may control responses with a very wide range of procedures (very wide relative to the range of most psychological theories). The new evidence also suggests that distinct serial processes can be part of a more elaborate mechanism and that they not only condense (input more diverse than output) but also elaborate (output more diverse than input).

Why is generality important? For one thing, it provides motivation. Many scientists care about the generality of what they study. For example, a mathematician who studies computer algorithms told me that demonstrations of the equivalence of different problems were very important to her because they meant that a solution to the problem that she was working on would have far-reaching implications. "It shows you're not just working on a detail," she said. The discovery of a connection between animal free-operant behavior and human cognition (as revealed in RT experiments) suggests that those who study animal free-operant behavior are not just working on a detail. In addition, generality provides "model systems"new situations for studying old problems. We can surely learn more about distinct serial processes, especially their neural basis, if we can study them in animals.

\section{REFERENCES}

AcostA, E., \& Simon, J. R. (1976). The effect of irrelevant information on the stages of processing. Journal of Motor Behavior, 8, 181-187.

AsHBY, F. G. (1982). Deriving exact predictions from the cascade model. Psychological Review, 89, 599-607.

Ayala, F. J., \& Kiger, J. A., JR. (1984). Modern genetics (2nd ed.). Menlo Park, CA: Benjamin/Cummings.

AzrIN, N. H., \& Holz, W. C. (1961). Punishment during fixed-interval reinforcement. Journal of the Experimental Analysis of Behavior, 4, 343-347.

BAtESON, W. (1913). Mendel's principles of heredity. Cambridge, England: Cambridge University Press.

BENTLEY, D., \& Konishi, M. (1978). Neural control of behavior. Annual Review of Neuroscience, 1, 35-59.

Bernstein, I. H., Chu, P. K., \& Briggs, P. (1973). Stimulus intensity and foreperiod effects in intersensory facilitation. Quarterly Journal of Experimental Psychology, 25, 171-181.

Biederman, I., \& KAPLAN, R. (1970). Stimulus discriminability and
S-R compatibility: Evidence for independent effects on choice reaction time. Journal of Experimental Psychology, 86, 434-439.

Blackman, A. R. (1975). Test of the additive-factor method of choice reaction time. Perceptual \& Motor Skills, 41, 607-613.

Blakemore, C., \& Campbell, F. W. (1969). On the existence of neurones in the human visual system selectively sensitive to the orientation and size of retinal images. Journal of Physiology, 203, 237-260.

BLOUGH, D. S. (1963). Interresponse time as a function of continuous variables: A new method and some data. Journal of the Experimental Analysis of Behavior, 6, 237-246.

Blough, D. S. (1965). Definition and measurement in generalization research. In D. I. Mostofsky (Ed.), Stimulus generalization (pp. 3037). Stanford, CA: Stanford University Press.

BLough, D. S. (1967). Stimulus generalization as signal detection in pigeons. Science, 158, 940-941.

BLOUGH, D. S. (1972). Recognition by the pigeon of stimuli varying in two dimensions. Journal of the Experimental Analysis of Behavior, $18,345-367$.

BLOUGH, D. S. (1977). Visual search in the pigeon: Hunt and peck method. Science, 196, 1013-1014.

BLough, D. S. (1978). Reaction times of pigeons on a wavelength discrimination task. Journal of the Experimental Analysis of Behavior, 30, 163-167.

BLough, D. S., \& BLough, P. (1977). Animal psychophysics. In W. K. Honig \& J. E. R. Staddon (Eds.), Handbook of operant behavior (pp. 514-539). Englewood Cliffs, NJ: Prentice-Hall.

BRACEY, G. W. (1969). Two operations in character recognition: A partial replication. Perception \& Psychophysics, 6, 357-360.

BroAdBENT, D. E. (1984a). The Maltese cross: A new simplistic model for memory. Behavioral \& Brain Sciences, 7, 55-68.

BroadBEnt, D. E. (1984b). Modules in models of memory. Behavioral \& Brain Sciences, 7, 86-94.

Castellucci, V., \& Kandel, E. R. (1974). A quantal analysis of the synaptic depression underlying habituation of the gill-withdrawal reflex in Aplysia. Proceedings of the National Academy of Sciences (U.S.A.), 71, 5004-5008.

Checkosky, S. F., \& Whitlock, D. (1973). Effects of pattern goodness on recognition time in a memory search task. Journal of Experimental Psychology, 100, 341-348.

CLARK, F. C. (1958). The effect of deprivation and frequency of reinforcement on variable-interval responding. Joumal of the Experimental Analysis of Behavior, 1, 221-228.

Commons, M. L. (1981). Preface. In M. L. Commons \& J. A. Nevin (Eds.), Quantitative analyses of behavior: Vol. 1. Discriminative properties of reinforcement schedules ( $\mathrm{pp}$. xvii-xix). Cambridge, MA: Ballinger.

CORBIT, J. D., \& LUSCHEI, E. S. (1969). Invariance of the rat's rate of drinking. Journal of Comparative \& Physiological Psychology, 69, 119-125.

Correns, C. (1966). G. Mendel's law concerning the behavior of progeny of varietal hybrids. In C. Stern \& E. R. Sherwood (Eds.), The origin of genetics: A Mendel source book (pp. 119-132). San Francisco: W. H. Freeman. (Original work published 1900)

Cox, C., \& GABriel, K. R. (1982). Some comparisons of biplot display and pencil-and-paper E.D.A. methods. In R. L. Launer \& A. F. Siegel (Eds.), Modern data analysis (pp. 45-82). New York: Academic Press.

DE VRIES, H. (1966). The law of segregation of hybrids. In C. Stern \& E. R. Sherwood (Eds.), The origin of genetics: A Mendel source book (pp. 107-117). San Francisco: W. H. Freeman. (Original work published 1900)

DiNSMOOR, J. A. (1952). The effect of hunger on discriminated responding. Journal of Abnormal \& Social Psychology, 42, 67-72.

DUNCAN, E. M., \& MCFarLand, C. E., Jr. (1980). Isolating the effects of symbolic distance and semantic congruity in comparative judgments: An additive-factors analysis. Memory \& Cognition, 8, 612-622.

EDMonds, D. E., \& Gallistel, C. R. (1974). Parametric analysis of brain stimulation reward in the rat: III. Effect of performance variables on the reward summation function. Journal of Comparative \& Physiological Psychology, 87, 876-883.

EGETH, H. (1977). Atiention and preattention. In G. H. Bower (Ed.), 
The psychology of learning and motivation (Vol. 11, pp. 277-320) New York: Academic Press.

Emerson, J. D., Honglin, D. C., \& Kemprhorne, P. J. (1984). Leverage in least squares additive-plus-multiplicative fits for two-way tables. Jourmal of the American Statistical Association, 79, 329-335.

Emerson, J. D., \& Wong, G. W. (1985). Resistant nonadditive fits for two-way tables. In D. C. Hoaglin, F. Mosteller, \& J. W. Tukey (Eds.), Exploring data tables, trends, and shapes (pp. 67-124). New York: Wiley.

Fisher, R. A. (1966). Has Mendel's work been rediscovered? In C. Stern \& E. R. Sherwood (Eds.), The origin of genetics: A Mendel source book (pp. 139-172). San Francisco: W. H. Freeman. (Original work published 1936)

Flaherty, C. F. (1985). Animal learning and cognition. New York: Knopf.

Ford, B., \& BANKs, W. P. (1977). Perceptual differences between reading handwritten and typed words. Memory \& Cognition, 5, 630-635.

Freedman, D. A. (1983). Statistics and the scientific method (Tech. Rep. No. 19). Berkeley: University of California, Department of Statistics.

Frowein, H. W., \& SANDERS, A. F. (1978). Effects of visual stimulus degradation, S-R compatibility, and foreperiod duration on choice reaction time and movement time. Bulletin of the Psychonomic Society, 12, 106-108.

GALLISTEL, C. R. (1980). The organization of action: A new synthesis. Hillsdale, NJ: Erlbaum.

GARDNER, H. (1985). The mind's new science: A history of the cognitive revolution. New York: Basic Books.

GeschWIND, N. (1979). Specializations of the human brain. In The brain (pp. 105-117). San Francisco: W. H. Freeman.

Grbmon, J., ChURCh, R. M. (1984). Sources of variance in an information-processing theory of timing. In H. L. Roitblat, T. G. Bever, \& H. S. Terrace (Eds.), Animal cognition (pp. 465-488). Hillsdale, NJ: Erlbaum.

Glass, A. L., Hol.yoak, K. J., \& Santa, J. L. (1979). Cognition. Reading, MA: Addison-Wesley.

GreEN, D. M., Swets, J. A. (1974). Signal detection theory and psychophysics (2nd ed.). Huntington, NY: Robert E. Krieger.

Grice, G. R., NullmeYer, R., SPIKER, V. A. (1982). Human reaction time: Toward a general theory. Joumal of Experimental Psychology: General, 111, 135-153.

Hamming, R. W. (1962). Numerical methods for scientists and engineers. New York: McGraw-Hill.

HAMPel, F. R. (1973). Robust estimation: A condensed partial survey. Zeitschrift fur Wahrscheinlichkeitstheorie und verwandte Gebiete, 27, 87-104.

Hardzinski, M., \& Pachella, R. G. (1980). The manipulation of stimulus quality and the definition of stimulus encoding operations in memory scanning experiments. Perception \& Psychophysics, 27, 232-240.

Hearst, E., Koresko, M. B. (1968). Stimulus generalization and amount of prior training on variable-interval reinforcement. Journal of Comparative \& Physiological Psychology, 66, 133-138.

HeInemanN, E. G. (1974). Multimodal distributions of pigeon's reaction time. Bulletin of the Psychonomic Society, 3, 75-77.

Hoffman, H. S., SeArLe, J. L. (1965). Acoustic variables in the modification of startle reaction in the rat. Journal of Comparative \& Physiological Psychology, 60, 53-58.

Hofrman, H. S., \& WibLe, B. L. (1969). Temporal parameters in startie facilitation by steady background signals. Journal of the Acoustical Society of America, 45, 7-12.

HOFFMAN, H. S., \& WiBLe, B. L. (1970). Role of weak signals in acoustic startle. Journal of the Acoustical Society of America, 47, 489-497.

Holder, M. D., \& RoberTs, S. (1985). Comparison of timing and classical conditioning. Journal of Experimental Psychology: Animal Behavior Processes, 11, 172-193.

HuLL, C. L. (1943). Principles of behavior: An introduction to behavior theory. New York: Appleton-Century.

IsAAC, P. D. (1970). Linear regression, structural relations, and measurement error. Psychological Bulletin, 74, 213-218.
KIRN, J., \& FLoOdy, O. R. (1985). Differential effects of lesions in three limbic areas on ultrasound production and lordosis by female hamsters. Behavioral Neuroscience, 99, 1142-1152.

KisH, G. B. (966). Studies of sensory reinforcement. In W. K. Honig (Ed.), Operant behavior: Areas of research and application (pp. 109158). New York: Meredith.

KoNICK, D. S., \& ThomAs, D. R. (1968). Stimulus generalization following fixed interval training. Journal of Experimental Psychology, 77, 689-691.

KUfFler, S. W., Nicholls, J. G. (1976). From neuron to brain: A cellular approach to the function of the nervous system. Sunderland, MA: Sinauer.

KuPferman, I., \& Weiss, K. R. (1978). The command neuron concept. Behavioral \& Brain Sciences, 1, 3-39.

LandaUer, T, K., Didner, R. S., Fowlkes, E. B. (1975, April). Processing stages in word naming: Reaction time effects of letter degradation and word frequency. Paper presented at the meeting of the Eastern Psychological Association, New York.

LoFTus, G. (1985). Johannes Kepler's computer simulation of the universe: Some remarks about theory in psychology. Behavior Research Methods, Instruments, \& Computers, 17, 149-156.

LOGAN, G. D. (1978). Attention in character-classification tasks: Evidence for the automaticity of component stages. Joumal of Experimental Psychology: General, 107, 32-63.

MACKINTOSH, N. J. (1974). The psychology of animal learning. London: Academic Press.

MACKINTOSH, N. J. (1975). A theory of attention: Variations in the associability of stimuli with reinforcement. Psychological Review, 82, 276-298.

MAKI, W. S. (1979). Discrimination learning without short-term memory: Dissociation of memory processes in pigeons. Science, 204, 83-85.

MANDEL, J. (1971). A new analysis of variance model for non-additive data. Technometrics, 13, 1-18.

Marice, A. V., RoberTs, S., \& ChURCH, R. M. (1981). Methamphetamine and time estimation. Joumal of Experimental Psychology: Animal Behavior Processes, 7, 18-30.

Marsh, R. R., Hoffman, H. S., Stitt, C. L., Schwartz, G. M. (1975). The role of small changes in the acoustic environment in modifying the startle reflex. Joumal of Experimental Psychology: Animal Behavior Processes, 104, 235-244.

MCClell.AND, J. L. (1979). On the time relations of mental processes: An examination of systems of processes in cascade. Psychological Review, 86, 287-330.

MCNeIL, D. R., TukeY, J. W. (1975). Higher-order diagnosis of two-way tables, illustrated on two sets of demographic empirical distributions. Biometrics, 31, 487-510.

Meltzer, D., \& Brahlek, J. A. (1968). Quantity of reinforcement and fixed-interval performance. Psychonomic Science, 12, 207-208.

Meltzer, D., BRAHLEK, J. A. (1970). Quantity of reinforcement and fixed-interval performance: Within-subject effects. Psychonomic Science, 20, 30-31.

MENDEL, G. (1966). Experiments on plant hybrids. In C. Stern \& E. R. Sherwood (Eds.), The origin of genetics: A Mendel source book (pp. 1-48). San Francisco: W. H. Freeman. (Original work published 1865)

MeYer, D. E., Yantis, S., Osman, A. M., \& SMrTh, J. E. K. (1985). Temporal properties of human information processing: Tests of discrete versus continuous models. Cognitive Psychology, 17, 445-518.

MiLEs, R. C. (1956). The relative effectiveness of secondary reinforcers throughout deprivation and habit-strength parameters. Joumal of Comparative \& Physiological Psychology, 49, 126-130.

Miller, J. C., \& Pachella, R. G. (1973). Locus of the stimulus probability effect. Journal of Experimental Psychology, 101, 227-291.

Mosteller, F., \& TUKEY, J. W. (1977). Data analysis and regression: A second course in statistics. Reading, MA: Addison-Wesley.

Mulvanny, P. J. (1976). Pigeon discriminative reaction time: Comparison of incremental and all-or-none models. Unpublished doctoral dissertation, Brown University.

MURdock, B. B., JR. (1974). Human memory: Theory and data. Potomac, MD: Erlbaum.

Pachella, R. G. (1974). The interpretation of reaction time in 
information-processing research. In B. Kantowitz (Ed.), Human information processing: Tutorials in performance and cognition (pp. 41-82). Hillsdale, NJ: Erlbaum.

PaChella, R. G., Miller, J. O. (1976). Stimulus probability and same-different classification time. Perception \& Psychophysics, 19 , 29-34.

Penfield, W., \& Roberts, L. (1959). Speech and brain mechanisms. Princeton, NJ: Princeton University Press.

Perin, C. T. (1942). Behavior potentiality as a joint function of the amount of training and the degree of hunger at the time of extinction. Journal of Experimental Psychology, 30, 93-113.

PIETERS, J. P. M. (1983). Sternberg's additive factor method and underlying psychological processes: Some theoretical considerations. Psychological Bulletin, 93, 411-426.

Posner, M. I., Lewis, J. L., \& Conrad, C. (1972). Component processes in reading: A performance analysis. In J. F. Kavanagh \& I. G. Mattingly (Eds.), Language by ear and by eye (pp. 159-192). Cambridge, MA: MIT Press.

Poulton, E. C. (1968). The new psychophysics: Six models for magnitude estimation. Psychological Bulletin, 69, 1-19.

QUIGG, C., SchWITTERS, R. F. (1986). Elementary particle physics and the superconducting super collider. Science, 231, 1522-1527.

Riley, D. A., \& LEITH, C. R. (1976). Multidimensional psychophysics and selective attention in animals. Psychological Bulletin, 83, 138-160.

RitTer, W., Simson, R., VAUGHN, H. G., JR., \& MACHT, M. (1982). Manipulation of event-related potential manifestations of information processing stages. Science, 218, 909-911.

ROBERTS, S. (1981). Isolation of an internal clock. Joumal of Experimental Psychology: Animal Behavior Processes, 7, 242-268.

ROBERTS, S. (1982). Cross-modal use of an internal clock. Journal of Experimental Psychology: Animal Behavior Processes, 8, 2-22.

RoBerTs, S. (1983). Properties and function of an internal clock. In R. L. Mellgren (Ed.), Animal cognition and behavior (pp. 345-397). Amsterdam: North-Holland.

Roberts, S., \& CHURCH, R. M. (1978). Control of an internal clock. Journal of Experimental Psychology: Animal Behavior Processes, 4, 318-337.

ROBERTS, S., \& Holder, M. D. (1984). What starts an internal clock? Journal of Experimental Psychology: Animal Behavior Processes, 10, 273-296.

RoBerTS, S., \& Holder, M. D. (1985). Effect of classical conditioning on an internal clock. Journal of Experimental Psychology: Animal Behavior Processes, 11, 194-214.

RoGERS, T. B. (1974). An analysis of the stages underlying the process of responding to personality items. Acta Psychologica, 38, 205-213.

Rozin, P. (1965). Temperature independence of an arbitrary temporal discrimination in the goldfish. Science, 145, 561-563.

SANDERS, A. F. (1980). Stage analysis of reaction processes. In G. E. Stelmach \& J. Requin (Eds.), Tutorials in motor behavior (pp. 331354). Amsterdam: North-Holland.

SATINOFF, E. (1978). Neural organization and evolution of thermal regulation in mammals. Science, 201, 16-22.

SCHWARTZ, B. (1977a). Studies of operant and reflexive key pecks in the pigeon. Journal of the Experimental Analysis of Behavior, 27, 301-313.

ScHWARTZ, B. (1977b). Two types of pigeon key pecking: Suppression of long-but not short-duration key pecks by duration-dependent shock. Joumal of the Experimental Analysis of Behavior, 27, 393-398.

SchwarTZ, B., \& Williams, B. R. (1972). Two different kinds of key peck in the pigeon: Some properties of responses maintained by negative and positive response-reinforcer contingencies. Journal of the $E x-$ perimental Analysis of Behavior, 18, 201-216.

Schwartz, G. M., Hoffman, H. S., StitT, C. L., \& Marsh, R. R. (1976). Modification of the rat's acoustic startle response by antecedent visual stimulation. Journal of Experimental Psychology: Animal Behavior Processes, 2, 28-37.

SCHWEIKERT, R. (1985). Separable effects of factors on speed and accuracy: Memory scanning, lexical decision, and choice tasks. Psychological Bulletin, 97, 530-546.

SHANKWEILER, D. (1966). Effects of temporal-lobe damage on percep- tion of dichotically presented melodies. Journal of Comparative \& Physiological Psychology, 62, 115-119.

SHAw, M. L. (1980). Identifying attentional and decision-making components in information processing. In R. L. Nickerson (Ed.), Attention and performance VIII (pp. 277-296). Hillsdale, NJ: Erlbaum. Shwartz, S. P., Pomerantz, J. R., \& Egeth, H. E. (1977). State and process limitations in information processing: An additive factors analysis. Journal of Experimental Psychology: Human Perception \& Performance, 3, 402-410.

SkINNER, B. F. (1950). Are theories of learning necessary? Psychological Review, 57, 193-216.

SMITH, E. E. (1968). Choice reaction time: An analysis of the major theoretical positions. Psychological Bulletin, 69, 77-110.

SNEDECOR, G. W., CochRAN, W. G. (1980). Statistical methods (7th ed.). Ames: Iowa State University.

Stanners, R. F., Jastrzembski, J. E., \& Westbrook, A. (1975). Frequency and visual quality in a word-nonword classification task. Journal of Verbal Learning \& Verbal Behavior, 14, 259-264.

Stanovich, K. E., \& Pachella, R. G. (1977). Encoding, stimulusresponse compatibility, and stages of processing. Journal of Experimental Psychology: Human Perception \& Performance, 3, 411-421.

STERnBerg, S. (1963). Stochastic learning theory. In R. D. Luce, R. R. Bush, \& E. Galanter (Eds.), Handbook of mathematical psychology (Vol. 2, pp. 1-120). New York: Wiley.

STERNBERG, S. (1966). High-speed scanning in hurnan memory. Science, $153,652-654$.

STERNBERG, S. (1967). Two operations in character recognition: Some evidence from reaction-time measurements. Perception \& Psychophysics, 2, 45-53.

STERNBERG, S. (1969a). The discovery of processing stages: Extensions of Donders' method. In W. G. Koster (Ed.), Attention and performance II (pp. 276-315). Amsterdam: North-Holland. Reprinted from Acta Psychologica, 1979, 30, 276-315.

STERNBERG, S. (1969b). Memory-scanning: Mental processes revealed by reaction-time experiments. American Scientist, 4, 421-457.

STERNBerg, S. (1971, May). Decomposing mental processes with reaction-time data. Invited address given at the meeting of the Midwestern Psychological Association, Detroit.

STERNBERG, S. (1975). Memory scanning: New findings and current controversies. Quarterly Journal of Experimental Psychology, 27, 1-32.

STERNBERG, S. (1984). Stage models of mental processing and the additive-factor method. Behavioral \& Brain Sciences, 7, 82-84.

STERnberG, S., WrIGHT, C. E., KNoll, R. L., \& Monsell, S. (1980). Motor programs in rapid speech: Additional evidence. In R. A. Cole (Ed.), Perception and production of fluent speech (pp. 507-534). Hillsdale, NJ: Erlbaum.

STEVENS, J. C., \& MACK, J. D. (1959). Scales of apparent force. Journal of Experimental Psychology, 58, 405-413.

STEVENS, J. C., \& STEveNS, S. S. (1960). Warmth and cold: Dynamics of sensory intensity. Journal of Experimental Psychology, 60, 183-192.

STEVENS, S. S. (1966). On the operation known as judgment. American Scientist, 54, 385-401.

StitT, C. L., Hoffman, H. S. , MARsh, R. (1973). Modification of the rat's startle reaction by termination of antecedent acoustic signals. Joumal of Comparative \& Physiological Psychology, 84, 207-215.

STITT, C. L., HoffMaN, H. S., \& MARSH, R. (1976). Interaction versus independence of startle-modification processes in the rat. Journal of Experimental Psychology: Animal Behavior Processes, 2, 260-265.

StiTt, C. L., Hoffman, H. S., Marsh, R., Boskoff, K. J. (1974). Modification of the rat's startle reaction by an antecedent change in the acoustic environment. Journal of Comparative \& Physiological Psychology, 86, 826-836.

Sturtevant, A. H., \& Beadle, G. W. (1962). An introduction to genetics. New York: Dover. (Original work published 1939)

SutHerland, N. S., Mackintosh, N. J. (1971). Mechanisms of animal discrimination learning. New York: Academic Press.

SwETs, J. A. (Ed.). (1964). Signal detection and recognition by human observers: Contemporary readings. New York: Wiley.

SwETs, J. A. (1986). Form of empirical ROCs in discrimination and 
diagnostic tasks: Implications for theory and measurement of performance. Psychological Bulletin, 99, 181-198.

TAYLOR, D. A. (1976). Stage analysis of reaction time. Psychological Bulletin, 83, 161-191.

Teuber, H.-L. (1955). Physiological psychology. Annual Review of Psychology, 6, 267-296.

Tharp, V. K., Rundell, H. H., Lester, B. K., \& Williams, H. L. (1974). Alcohol and information processing. Psychopharmacologia, 40, 33-52.

TheiOs, J. (1973). Reaction time measurements in the study of memory processes: Theory and data. In G. H. Bower (Ed.), The psychology of learning and motivation (Vol. 7, pp. 43-85). New York: Academic Press.

Thomas, D. R., KING, R. A. (1959). Stimulus generalization as a function of level of motivation. Journal of Experimental Psychology, 57, 323-328

Tomie, A., DAvitT, G. A. , \& Thomas, D. R. (1973). Role of stimulus similarity in equivalence training. Journal of Experimental Psychology, 101, 146-150.

Townsend, J. T. (1984). Uncovering mental processes with factorial experiments. Journal of Mathematical Psychology, 28, 363-400

Treisman, M. (1963). Temporal discrimination and the indifference interval: Implications for a model of the "internal clock." Psychological Monographs: General \& Applied, 77(13, Whole No. 576).

TUKEY, J. W. (1977). Exploratory data analysis. Reading, MA: Addison-Wesley.

TUKEY, J. W. (1979). Robust techniques for the user. In R. L. Launer \& G. N. Wilkinson (Eds.), Robustness in statistics (pp. 103-106). New York: Academic Press.

Vaughn, H. G., JR., \& Ritrer, W. (1973). Physiologic approaches to the analysis of attention and performance. In S. Kornblum (Ed.), Attention and performance IV (pp. 129-154). New York: Academic Press.

WiCkelgren, W. A. (1977). Speed-accuracy tradeoff and information processing dynamics. Acta Psychologica, 41, 67-85.

WiCKelgren, W. (1981). Human learning and memory. Annual Review of Psychology, 32, 21-52.

Williams, S. B. (1938). Resistance to extinction as a function of the number of reinforcements. Journal of Experimental Psychology, 23, 506-521.

WILlows, A. O. D. (1967). Behavioral acts elicited by stimulation of single, identifiable brain cells. Science, 157, 570-574.

WILSON, M. (1957). Effects of circumscribed cortical lesions upon somesthetic and visual discrimination in the monkey. Journal of Comparative \& Physiological Psychology, 50, 630-635.

WILson, W. A., JR., \& Mishikin, M. (1959). Comparison of the effects of inferotemporal and lateral occipital lesions on visually guided behavior in monkeys. Journal of Comparative \& Physiological Psychology, 52, 10-17.

Wishner, J., Stein, M. K., \& Peastrel, A. L. (1978). Stages of information processing in schizophrenia: Sternberg's paradigm. In L. C. Wynne, R. L. Cromwell, \& S. Matthysse (Eds.), The nature of schizophrenia: New approaches to research and treatment (pp. 233243). New York: Wiley.

\section{APPENDIX A}

\section{How Models Were Fitted}

The fitted lines in Figure 2 (constrained to go through the origin) were generated by the APL program MULTFIT (McNeil $\&$ Tukey, 1975) with cutoff $=9$ and $\epsilon=.01$. This program fits a model of the form

$$
r(i, j)=a(i) * b(j),
$$

where $r(i, j)$ is the response rate at level $i$ of one factor and level $j$ of the other factor. The rest of the fits of this paper were gener- ated by the APL program ROMUFIT (McNeil \& Tukey, 1975), an extension of MULTFIT, using the same parameters (cutoff $=9$, etc.). ROMUFIT fits a model of the form

$$
r(i, j)=[a(i) * b(j)]+c(i) .
$$

When plotted as in Figure 4, the fitted rates will be linear, but will not necessarily pass through the origin. This program is also responsible for the fits shown in the rate-versus-part-of-fit graphs (e.g., Figure 13). Both programs work by iteration, adjusting a fit until a function of the residuals is minimized.

Using MULTFIT or ROMUFIT to fit a single line through a set of, say, six $(x, y)$ points involves treating the set of points as a $2 \times 6$ table, where the $x$ values are one row and the $y$ values the other row. MULTFIT and ROMUFIT have important advantages over least squares regression, the usual way of fitting a line. First, conventional regression makes the unrealistic (in this case) assumption that $x$ is measured with much less error than $y$ (e.g., Isaac, 1970). MULTFIT and ROMUFIT assume that both variables, $x$ and $y$, are measured with roughly equal error (variance), in the sense that both programs give the residuals produced by the $x$ values (one row) and the residuals produced by the $y$ values (the other row) equal weight in finding the fit. Second, with cutoff $=9$, both programs produced resistant fits, fits that are insensitive to changes in one or a few values, in contrast to least squares fits. In a number of cases (Figures 15, 16, 17, and 23), a multiplicative model fits well except for one or two points; a nonresistant fitting procedure would not have shown this as clearly (Emerson, Hoaglin, \& Kempthorne, 1984). For discussions of the value of resistant fits, see McNeil and Tukey (1975), Mosteller and Tukey (1977, chap. 10), Roberts and Holder (1985), and Tukey (1979). For other methods of fitting models of the sort used here, see Emerson and Wong (1985) and its references.

\section{APPENDIX B \\ Significance Tests}

\section{Does the Model Fit?}

To ask if the residuals from a fit are significantly different from zero, one needs to compute the fit using some of the data and then test the residuals generated by the rest of the data. The separation of fitting data from testing data makes the independence of the residuals used for testing more likely. To increase the power of the test, one can fit and get residuals from the data of each animal separately, then combine the results across animals (e.g., 2 residuals from each of 5 animals gives a sample of 10). If the model is reasonable, and the errors are independent, the residuals should be positive and negative equally often, or have a mean of zero.

The choice of points for fitting and testing depends on the pattern that one wants to detect. When the data are fitted with a single straight line (e.g., Figures 4,11 ), the most likely systematic deviation is probably curvature. If there are, say, four points along the line (e.g., Figure 4), to test for curvature, one can fit a line to the first and last points (or the middle two points) and ask if the middle two points (or the first and last points) are reliably different from zero. To test for S-shapedness-not done here - one can fit a line to the first and third (or to the second and fourth) points, and ask if the second and fourth (or first and third) points are reliably nonzero. 
The data of individual subjects were available for three experiments with multiplicative factors: Meltzer and Brahlek (1970, Figure 4); Rozin (1965, Figure 19); and Roberts (1983, Figures 18 and 24). They were examined for curvature. For the Meltzer and Brahlek data (4 rats), with four points, a line was fit to the first and last points and the residuals from the middle two points were tested. This produced eight residuals, not reliably different from zero $[W(8)=21$, two-tailed $p>.20]$. The Rozin data ( 3 goldfish) had six points, so a line was fit to the first two and last two points and the middle two points were tested. The residuals were not different from zero $[W(6)=15, p>.10]$. The Roberts data (6 rats) also had six points, so the same points were used. In none of the four cases were the residuals nonzero [measuring lever responses and examining the effect of signal, $W(12)=20, p>.20$; measuring chain responses and examining the effect of signal, $W(12)=42, p>.10$; examining the effect of response during the lever signal, $W(12)=18, p>.20$; examining the effect of response during the chain signal, $W(12)$ $=36, p>.10]$. In summary, none of the three experiments observed reliable curvature.

\section{Is an Intercept Nonzero?}

When rates are plotted against rates (e.g., Figure 4), a nonzero intercept suggests the presence of noise. Estimates of the reliability of intercepts in this paper come from two sources: (1) When the data for individual animals were available, an intercept was computed for each animal separately, using the fitting procedure (ROMUFIT) described above. The $t$ value of the mean over the individual intercepts was the test statistic. (2) When only the group data were available, the $t$ value for a nonzero intercept (Snedecor \& Cochran, 1980, p. 173) was computed in two ways: for the regression of $y$ on $x$ and for the regression of $x$ on $y$. Then the minimum of the two $t$ values (called "min $t$ ") was used as the test statistic. Because the correlation between $x$ and $y$ was high (more than .99) in almost all of the examples, there was never a difference in significance between the two values.

A more systematic approach would be to fit a succession of models, differing in their complexity. One would start with Model 1 (no noise); if the deviations from the fit were systematic, one would fit Model 1 amended to include an additive constant (noise present, unchanged by both factors). If this model produced systematic deviations, one would try Model 2 (noise present, changed by one factor). And so on. But this approach requires having enough independent replications (e.g., different animals) to have a reasonable chance of finding reliable lack of fit.

\section{APPENDIX C}

\section{Predictions of AND-Gate Model With Long Hold and Random Pulse Generation}

This appendix derives the prediction shown in Figure 9 for the case of the AND-gate model that assumes that (1) the hold of the AND gate is unlimited and (2) the two processes that send input to the AND gate generate pulses at random intervalsthat is, the probability of a pulse from either process is constant with time.

We seek the mean interval between pulses generated by the AND gate. Let $t$ be the time since the last AND-gate pulse. Let $p_{x}(t)$ be the probability that the first pulse occurs at time $t$-the probability density function-with $x=1,2$, or $g$, for the two processes (1 and 2) and the AND gate $(g)$. Let $P_{x}(t)$ be the probability that a pulse is generated by time $t$-the cumulative distribution function. By definition, we have

$$
P_{g}(t)=p_{1}(t) P_{2}(t)+p_{2}(t) P_{1}(t),
$$

because the gate will respond at time $t$ if the first process responds at time $t$ and the second process responds by time $t$, or vice versa. Let $r_{1}$ and $r_{2}$ be the rates (pulses per unit time) of the two processes. Because responding happens randomly,

$$
p_{1}(t)=r_{1} e^{-r_{1} t}
$$

Therefore,

$$
P_{1}(t)=\int_{0}^{t} p_{1}(x) d x=1-e^{-r_{1} t}
$$

And likewise for the second process. Therefore, the mean interpulse interval of the gate,

$$
\begin{aligned}
\int_{0}^{\infty} t p_{2}(t) d t & =\int_{0}^{\infty} t r_{1} e^{-r_{1} t}+t r_{2} e^{-r_{2} t}-t\left(r_{1}+r_{2}\right) e^{-\left(r_{1}+r_{2}\right) t} d t \\
& =\left(1 / r_{1}\right)+\left(1 / r_{2}\right)-\left(1 / r_{1}+r_{2}\right)
\end{aligned}
$$

Because the mean interpulse interval equals (total time)/(total responses), the reciprocal of the mean interpulse interval is the pulse rate.

\section{APPENDIX D}

\section{Comparison of Two Types of Graph}

Two types of graph-rate versus rate (RvR; e.g., Figure 2) and rate versus part of fit (RvPOF; e.g., Figure 13)-are used here to show the multiplicative fits because each type of graph is better in some ways than the other type.

\section{How are RvR Graphs Better than RvPOF Graphs?}

They are (1) more familiar, (2) much easier to make, and (3) much easier to understand, at least initially. (4) They do not depend on the method of fitting. (5) The graph of a $2 \times n$ experiment involves only one line, making it easy to compare the data from two experiments (e.g., Figure 4) or the predictions of a number of theories (e.g., Figure 9). (6) Perhaps most important, if all possible pairs of levels are plotted (e.g., in Figure 2, VI 1 vs. VI 2, VI 1 vs. VI 3, and VI 2 vs. VI 3), RvR graphs allow one to search for multiplicative relations within all possible subsets of the data, because the eye can detect linearity within any subset of points. This feature was important in analyzing the Roberts data (Figures 18 and 24).

\section{How are RvPOF Graphs Better than RvR Graphs?}

(1) When both factors have more than two levels, RvR graphs become cumbersome (because all possible pairs of levels are plotted) or potentially misleading (because some possible pairs are not plotted). In contrast, RvPOF graphs handle this situation easily. (2) RvPOF graphs are much closer than RvR graphs to the usual ways that fits are shown (e.g., by Sternberg, 1969a), where each point represents one residual. This representation makes it easier to decide if a certain residual is unusually large, and easier to judge the size of the residuals (unexplained vari- 
ance) relative to the size of the fit (explained variance). (3) As explained in the main text, RvPOF graphs make it easier to assess noise - to decide what amounts of noise are consistent with the data.

A third type of graph, with some of the best and worst features of the first two types, is the biplot (e.g., Cox \& Gabriel, 1982) from a principal-components analysis. The main use of biplots will probably be for exploration of the data. At the moment, biplots are more difficult to make than the other two types, and much more difficult to explain.

(Manuscript received March 27, 1984; revision accepted for publication December $8,1986$. 